CRYSTALLOGRAPHIC COMMUNICATIONS

ISSN 2056-9890

Received 27 September 2020

Accepted 5 October 2020

Edited by M. Zeller, Purdue University, USA

Keywords: crystal structure; nickel; pincer ligand; $\mathrm{N}$-donors; carbon monoxide.

CCDC references: 2035500; 2035499; 2035498; 2035497

Supporting information: this article has supporting information at journals.iucr.org/e

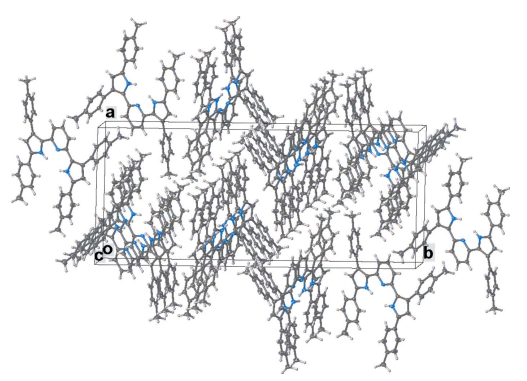

OPEN Ә ACCESS

\section{Nickel(II) carbonyl, ammonia, and acetonitrile complexes supported by a pyridine dipyrrolide pincer ligand}

\author{
H. V. Rasika Dias* and Abhijit Pramanik
}

Department of Chemistry and Biochemistry, The University of Texas at Arlington, Arlington, Texas 76019, USA.
*Correspondence e-mail: dias@uta.edu

The synthesis, isolation and crystal structures of nickel(II) carbonyl, acetonitrile and ammonia complexes supported by a dianionic, pyridine dipyrrolide pincer ligand $\left[\mathrm{pyrr}_{2} \mathrm{py}\right]^{2-}$, namely, carbonyl[2,2'-(pyridine-2,6-diyl)bis(3,5-di- $p$-tolylpyrrolido- $\kappa N)$ ]nickel(II), [ $\left.\mathrm{Ni}\left(\mathrm{C}_{41} \mathrm{H}_{33} \mathrm{~N}_{3}\right)(\mathrm{CO})\right]$, ammine[2,2'-(pyridine-2,6-diyl)bis(3,5-di- $p$-tolylpyrrolido- $\kappa N)]$ nickel(II), $\left[\mathrm{Ni}\left(\mathrm{C}_{41} \mathrm{H}_{33} \mathrm{~N}_{3}\right)\left(\mathrm{NH}_{3}\right)\right]$, and (acetonitrile- $\kappa N)\left[2,2^{\prime}\right.$-(pyridine-2,6-diyl)bis(3,5-di- $p$-tolylpyrrolido- $\left.\left.\kappa N\right)\right]$ nickel(II), $\left[\mathrm{Ni}\left(\mathrm{C}_{41} \mathrm{H}_{33} \mathrm{~N}_{3}\right)\left(\mathrm{CH}_{3} \mathrm{CN}\right)\right]$, as well as the free ligand 2,6-bis(3,5-di- $p$-tolylpyrrol-2yl)pyridine, $\mathrm{C}_{41} \mathrm{H}_{35} \mathrm{~N}_{3}$ or $\left[\mathrm{pyrr}_{2} \mathrm{py}\right] \mathrm{H}_{2}$ are reported. The nickel complexes are four-coordinate and adopt a square-planar geometry. The $\mathrm{CO}$ stretch of the nickel-bound carbon monoxide ligand of [pyrr $\left.{ }_{2} \mathrm{py}\right] \mathrm{Ni}(\mathrm{CO})$ has been observed at $2101 \mathrm{~cm}^{-1}$. The ammonia and acetonitrile complexes, $\left[\operatorname{pyrr}_{2}\right.$ py] $\mathrm{Ni}\left(\mathrm{NH}_{3}\right)$ and $\left[\right.$ pyrr $_{2}$ py] $\mathrm{Ni}(\mathrm{NCMe})$ feature all-nitrogen coordination spheres around nickel consisting of different $\mathrm{N}$-donor ligand types.

\section{Chemical context}

Pincer ligands were first introduced by Moulton and Shaw in 1976 (Moulton \& Shaw, 1976). They are utilized widely as auxiliary ligands to produce transition-metal complexes useful in a range of applications including catalysis (Alig et al., 2019; Peris \& Crabtree, 2004, 2018; Piccirilli et al., 2020; Albrecht \& van Koten, 2001). There are several pincer-ligand varieties in the literature ranging from those featuring both symmetric and non-symmetric flanking arms, $\mathrm{P}-, \mathrm{N}-, \mathrm{O}-, \mathrm{S}-$ and $\mathrm{C}$ - donor sites, as well as neutral, mono, di- and trianionic systems. Monoanionic, carbon-centered (e.g., from phenyl) ligands with $\mathrm{P}$ - or $\mathrm{N}$-donors at the flanking arms are more common among pincers (Peris \& Crabtree, 2018). These tridentate ligands are particularly interesting for their ability to preferentially occupy the meridional coordination sites on a metal ion.

We have been working on tridentate, nitrogen-based ligands such as tris(pyrazolyl)borates with a preference for facial coordination for several years (Dias \& Lovely, 2008; Dias et al., 1995, 1996; Dias \& Lu, 1995). This paper describes results from our efforts to expand the ligand repertoire to include tridentate ligands with a preference for meridional geometry (Adiraju et al., 2020) at transition-metal ions in our laboratory. In particular, we describe the synthesis and use of a pyridine dipyrrolide pincer ligand bearing tolyl substituents, (Pramanik et al., 2014; Pramanik, 2015) and its chemistry with nickel(II) featuring $\mathrm{CO}, \mathrm{NH}_{3}$ and $\mathrm{NCMe}$ molecules (Fig. 1). The pyridine dipyrrolide is a particularly attractive ligand framework, as several examples of pyridine dipyrrolide 
pincers with different substituents on the ligand backbone (e.g., $\mathrm{Me}, t$-Bu, $\mathrm{Ph}, \mathrm{Mes}$ ) are known and have already been successfully used with both early and late transition-metal ions such as Ti (Zhang et al., 2016), Zr (Zhang et al., 2016, 2020), Cr (Gowda et al., 2018), Mo (Gowda et al., 2018), Fe (Sorsche et al., 2020; Hakey et al., 2019), Co (Grant et al., 2016), Pt (Komine et al., 2014), Pd (Yadav et al., 2018) and Zn (Komine et al., 2014) to form well-defined metal complexes.

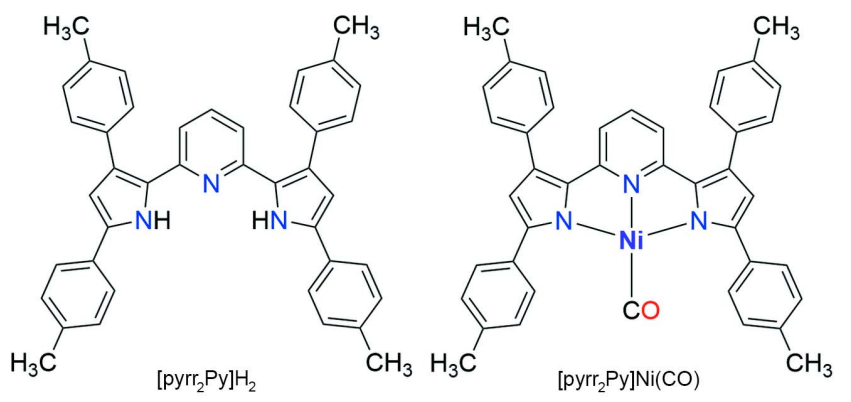

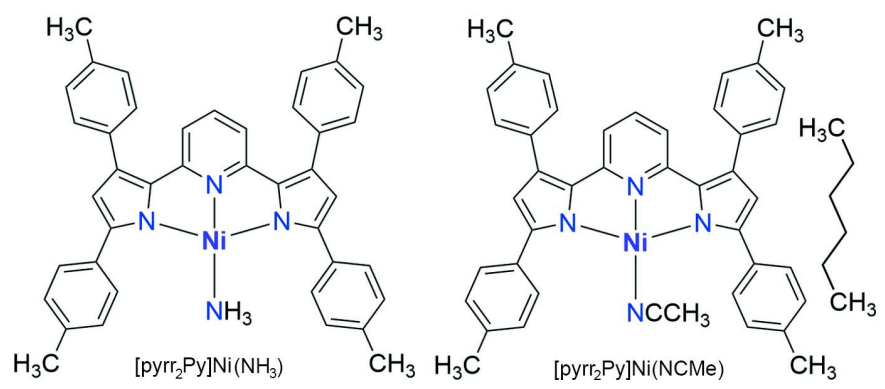

\section{Structural commentary}

The free ligand $\left[\mathrm{pyrr}_{2} \mathrm{Py}\right] \mathrm{H}_{2}$ is monomeric and crystallizes with both pyrrole nitrogen atoms facing the center of the coordination pit, well situated for metal-ion coordination (Fig. 2). This is different from the structure observed with the $t$-butyl substituted pincer analog (VIWSOL; Komine et al., 2014) in which one pyrrole $\mathrm{N}-\mathrm{H}$ bond is directed outward to form a hydrogen bond with a lattice acetonitrile molecule. The pyrrole and pyridine moieties are essentially coplanar. The nickel(II) carbonyl complex [pyrr $\left.{ }_{2} \mathrm{Py}\right] \mathrm{Ni}(\mathrm{CO})$ was synthesized from the in situ-generated potassium salt $\mathrm{K}_{2}\left[\mathrm{pyrr}_{2} \mathrm{Py}\right]$ and $\mathrm{Ni}(\mathrm{OTf})_{2}$ in the presence of carbon monoxide. The important

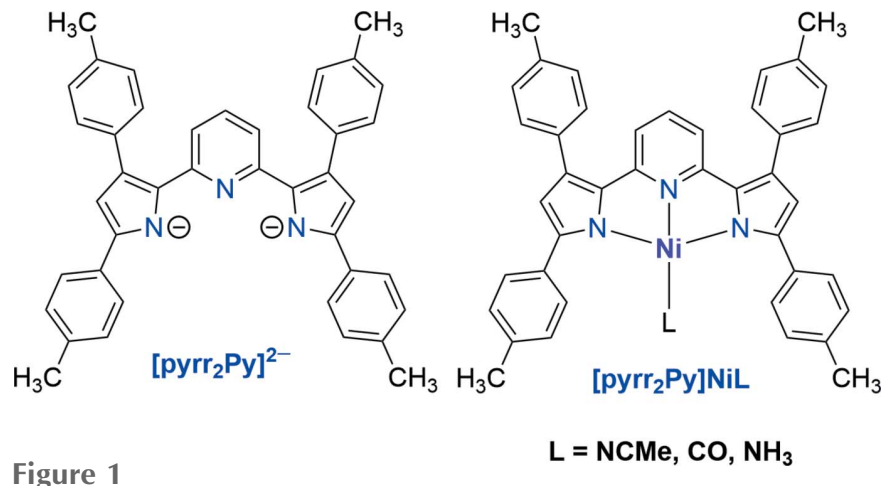

The dianionic, pyridine dipyrrolide pincer ligand $\left[\mathrm{pyrr}_{2} \mathrm{Py}\right]^{2-}$ and the nickel(II) complexes.

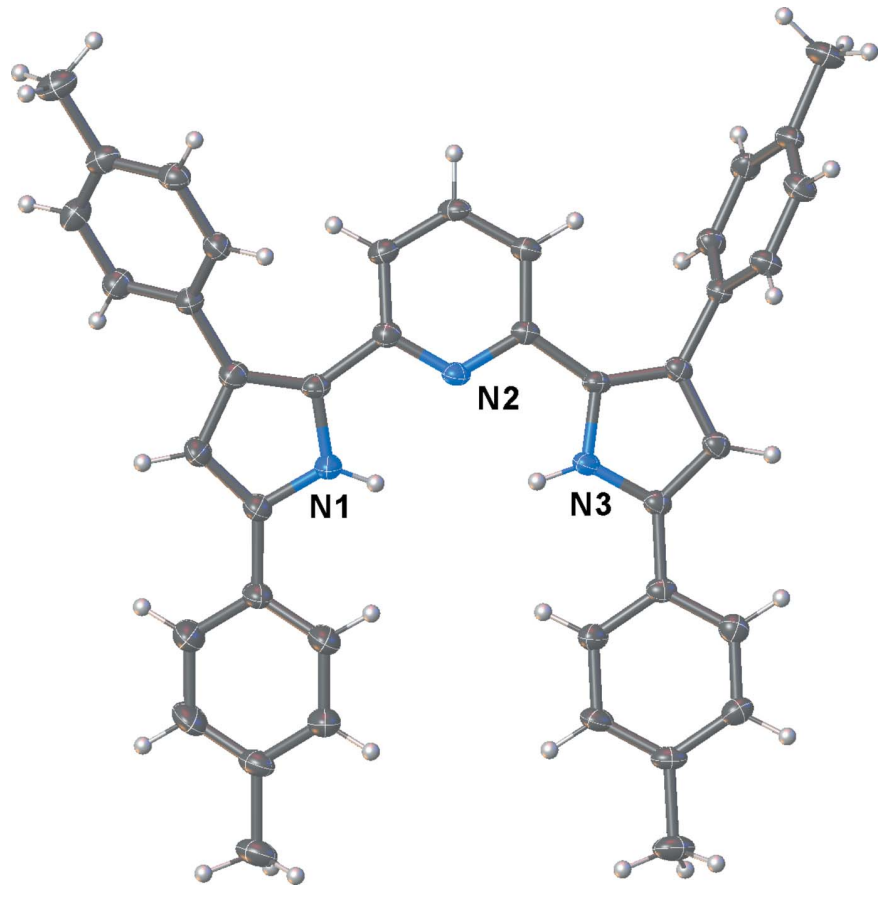

Figure 2

Molecular structure of $\left[\mathrm{pyrr}_{2} \mathrm{Py}\right] \mathrm{H}_{2}$ with displacement ellipsoids drawn at the $50 \%$ probability level.

CO stretch of this molecule is observed at $2101 \mathrm{~cm}^{-1}$, which is only slightly lower than that of free CO $\left(2143 \mathrm{~cm}^{-1}\right)$, indicating relatively weak $\mathrm{Ni} \rightarrow \mathrm{CO} \pi$-backbonding. The nickel(I) tris(pyrazolyl)borate complex $\left[\mathrm{HB}(3-\mathrm{Ph}, 5-\mathrm{MePz})_{3}\right] \mathrm{Ni}(\mathrm{CO})$ for comparison displays its $\mathrm{CO}$ stretch at $2003 \mathrm{~cm}^{-1}$ (Abubekerov et al., 2016). The X-ray crystal structure shows that the pincer complex $\left[\operatorname{pyrr}_{2} \mathrm{Py}\right] \mathrm{Ni}(\mathrm{CO})$ is a monomeric, square-planar complex (Fig. 3). The carbonyl moiety sits

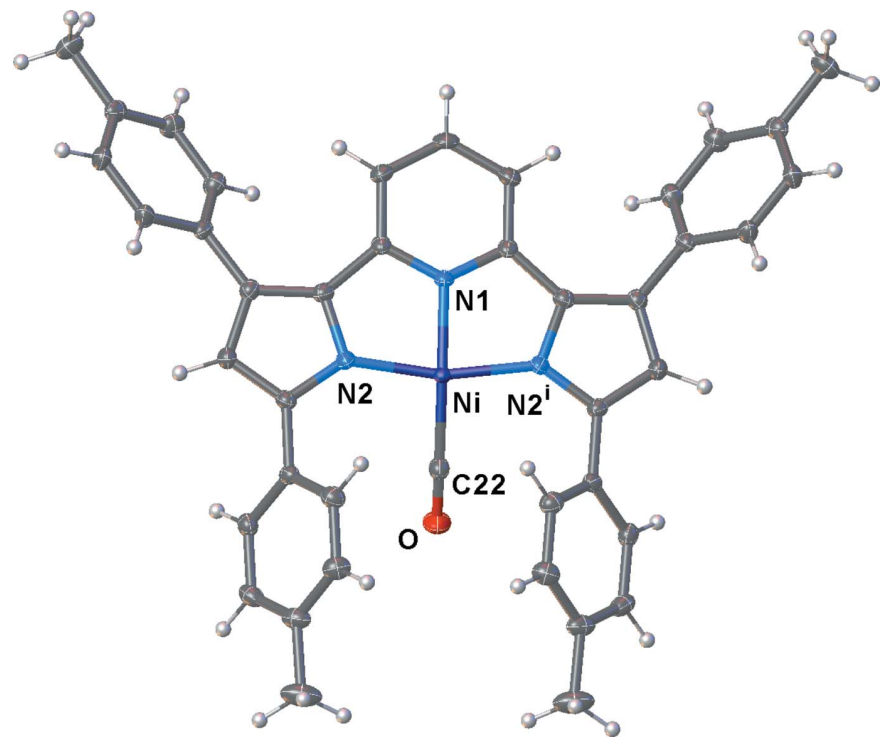

Figure 3

Molecular structure of $\left[\mathrm{pyrr}_{2} \mathrm{Py}\right] \mathrm{Ni}(\mathrm{CO})$ with displacement ellipsoids drawn at the $50 \%$ probability level. Symmetry code: (i) $1+x, \frac{3}{2}-y, \mathrm{z}$. 


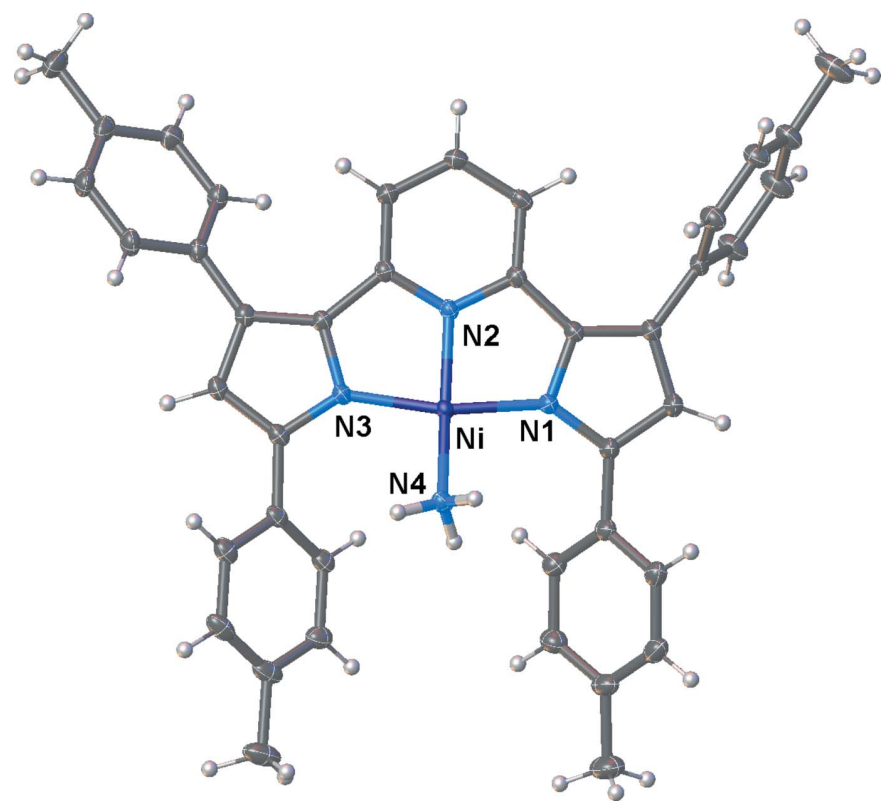

Figure 4

Molecular structure of $\left[\operatorname{pyrr}_{2} \mathrm{Py}\right] \mathrm{Ni}\left(\mathrm{NH}_{3}\right)$ with displacement ellipsoids drawn at the $50 \%$ probability level.

above the ligand plane, as is evident from the $\mathrm{N} 1-\mathrm{Ni}-\mathrm{C} 22$ angle of $160.41(13)^{\circ}$. The $\mathrm{Ni}-\mathrm{C} 22$ distance of 1.809 (3) $\AA$ is significantly longer than the corresponding distance of 1.766 (4) $\AA$ in $\left[\mathrm{HB}(3-\mathrm{Ph}, 5-\mathrm{MePz})_{3}\right] \mathrm{Ni}(\mathrm{CO})$, which is a tetrahedral nickel complex (ENUROW; Abubekerov et al., 2016). The $\mathrm{Ni}-\mathrm{N}($ pyrr $)($ pyrr $=$ pyrrolide $)$ distances of 1.8667 (18) and 1.8666 (18) $\AA$ are not significantly different from the $\mathrm{Ni}-$ $\mathrm{N}$ (pyridine) separation of 1.853 (3) $\AA$.

Compounds $\left[\mathrm{pyrr}_{2} \mathrm{Py}\right] \mathrm{Ni}\left(\mathrm{NH}_{3}\right)$ and $\left[\mathrm{pyrr}_{2} \mathrm{Py}\right] \mathrm{Ni}(\mathrm{NCMe})$ are also four-coordinate nickel(II) complexes with square-planar

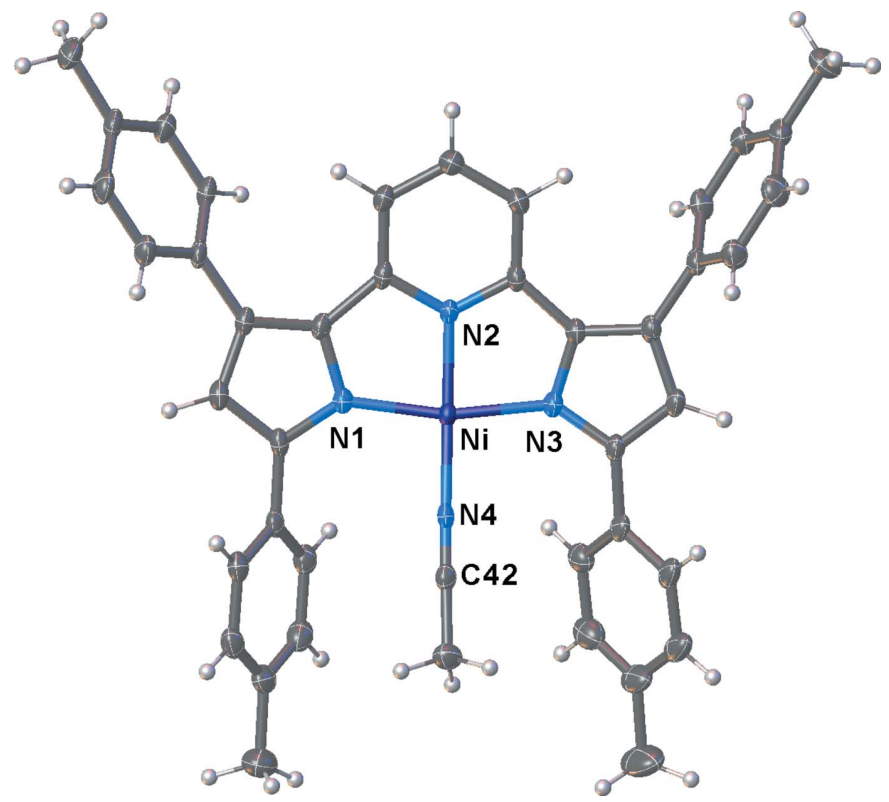

Figure 5

Molecular structure of [pyrr $\left.{ }_{2} \mathrm{Py}\right] \mathrm{Ni}(\mathrm{NCMe})$ with displacement ellipsoids drawn at the $50 \%$ probability level. A disordered hexane molecule has been omitted for clarity. metal sites (Figs. 4 and 5, respectively). They have all nitrogen coordination spheres at nickel, but with an interesting variety of donor sites ranging from $s p$ to $s p^{3}$-hybridized nitrogen atoms, as well as neutral and formally anionic $\mathrm{N}$-centers. Both the $\mathrm{NH}_{3}$ and $\mathrm{NCMe}$ ligands bend out of the [pyrr $2 \mathrm{Py}$ ] ligand plane as evident from the $\mathrm{N} 2-\mathrm{Ni}-\mathrm{N} 4$ angles of 162.16 (5) and $168.09(10)^{\circ}$, respectively, for the two complexes. The Ni$\mathrm{N} 1$ and $\mathrm{Ni}-\mathrm{N} 3$ bond distances of $\left[\mathrm{pyrr}_{2} \mathrm{Py}\right] \mathrm{Ni}\left(\mathrm{NH}_{3}\right)$ are 1.8858 (10) and $1.8876(10) \AA$, respectively. These values are marginally smaller than the corresponding distances of [pyrr 2 Py] Ni(NCMe) [1.896 (2) and 1.906 (2) $\AA$ ] . The Ni-N2 distances (to the pyridine moieties) at $1.8490(10)$ and 1.846 (2) $\AA$ are similar in the two adducts, but they are both much shorter than the $\mathrm{Ni}-\mathrm{N}$ (pyrr) distances noted above. The $\mathrm{Ni}-\mathrm{N}$ bond distance to the $\mathrm{NH}_{3}$ and $\mathrm{NCMe}$ ligands in $\left[\operatorname{pyrr}_{2} \mathrm{Py}\right] \mathrm{Ni}\left(\mathrm{NH}_{3}\right)$ and $\left[\mathrm{pyrr}_{2} \mathrm{Py}\right] \mathrm{Ni}(\mathrm{NCMe})$ are 1.9291 (11) and 1.861 (2) $\AA$, respectively. These are bonds to $s p^{3}$ and $s p$ hybridized nitrogen sites, respectively, and therefore the longer distance for the former is not unusual. Four-coordinate nickel-ammonia complexes are rare and there is one example in the CSD (PEWROZ; Tapper et al., 1993), and that has an $\mathrm{Ni}-\mathrm{N}\left(\mathrm{H}_{3}\right)$ distance of $1.912 \AA$.

\section{Supramolecular features}

Important intermolecular contacts and a packing diagram of $\left[\right.$ pyrr $\left._{2} \mathrm{Py}\right] \mathrm{H}_{2}$ are shown in Fig. 6 and Fig. S1 in the supporting information. Neighboring molecules of $\left[\mathrm{pyrr}_{2} \mathrm{Py}\right] \mathrm{H}_{2}$ show $\pi-\pi$ contacts between pyrrole and pyridine groups (the closest separation is $3.21 \AA$ ) as well as $\mathrm{C}($ arene $)-\mathrm{H} \cdots$ arene contacts. The complex $\left[\mathrm{pyrr}_{2} \mathrm{Py}\right] \mathrm{Ni}(\mathrm{CO})$ does not show extensive intermolecular interactions apart from $\mathrm{NiCO} \cdots \mathrm{H}-\mathrm{C}($ arene $)$ contacts between the carbonyl moieties and hydrogen atoms of neighboring arene as illustrated in Fig. 7 and Fig. S2. In the structure of $\left[\mathrm{pyrr}_{2} \mathrm{Py}\right] \mathrm{Ni}\left(\mathrm{NH}_{3}\right)$, the arene groups interact with neighboring molecules via the ammonia hydrogen atoms (see Fig. 8 and Fig. S3). In $\left[\mathrm{pyrr}_{2} \mathrm{Py}\right] \mathrm{Ni}(\mathrm{NCMe})$, the hexane molecules in the lattice occupy regions surrounded by tolyl substituents. The major intermolecular interactions are

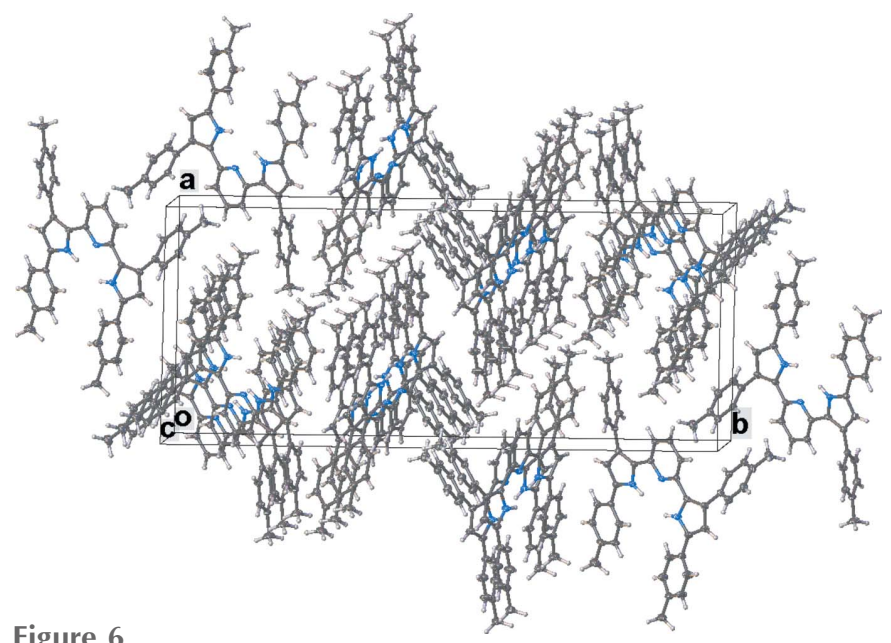

The crystal packing of $\left[\mathrm{pyrr}_{2} \mathrm{Py}\right] \mathrm{H}_{2}$. 


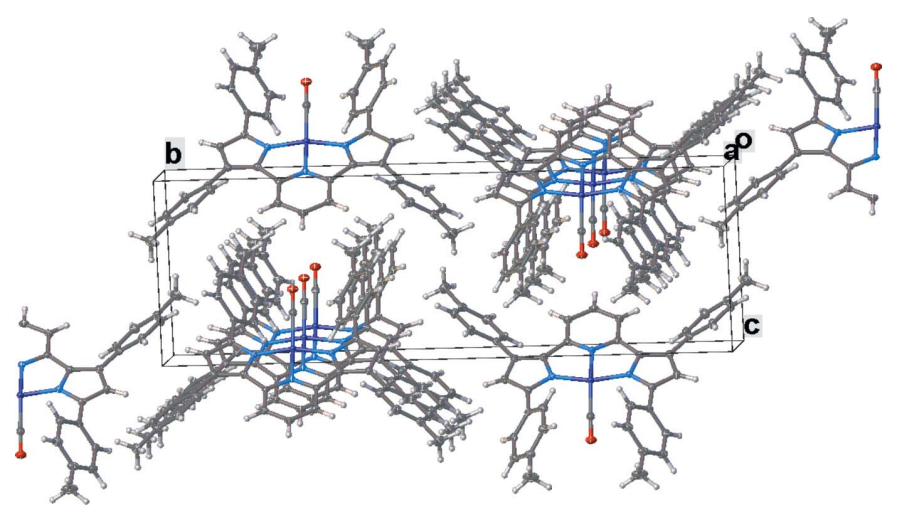

Figure 7

The crystal packing of $\left[\right.$ pyrr $\left._{2} \mathrm{Py}\right] \mathrm{Ni}(\mathrm{CO})$.

between arenes and the hydrogen atoms of the acetonitrile moieties. The resulting packing diagram is shown in Fig. 9 and Fig. S4.

\section{Database survey}

A search of the Cambridge Structural Database for related pyridine dipyrrolide complexes involving transition-metal ions

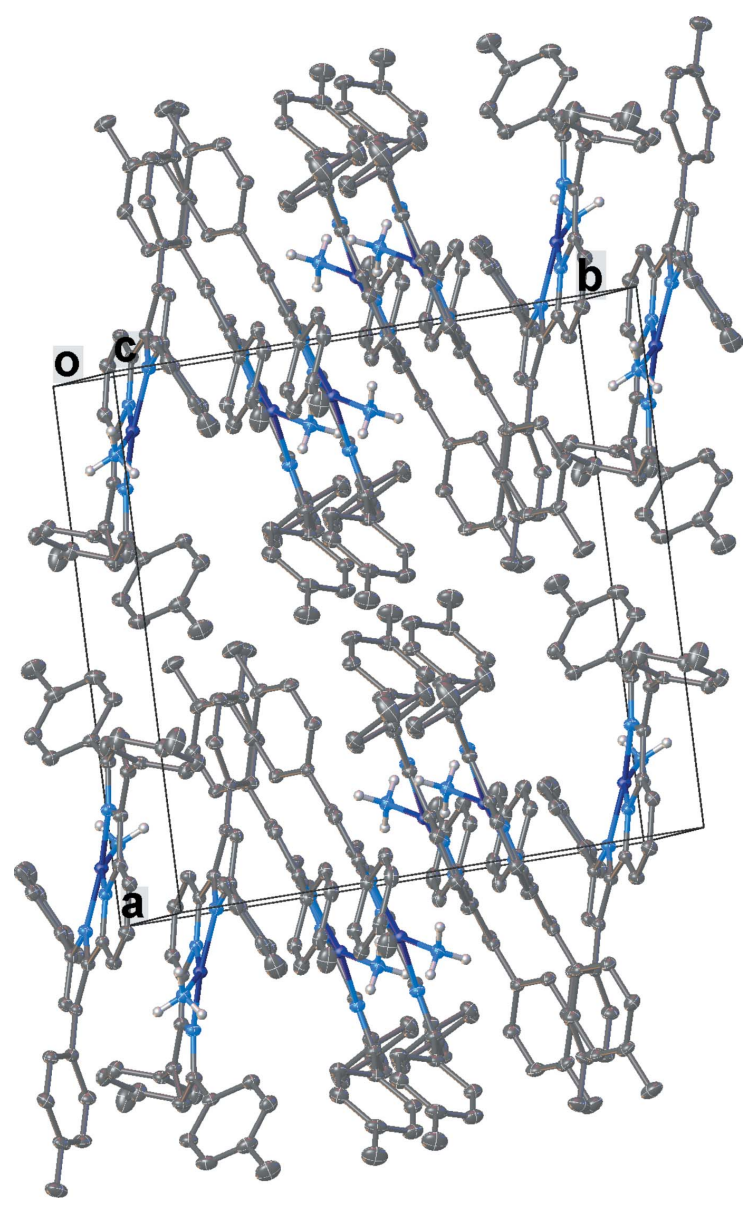

Figure 8

The crystal packing of $\left[\mathrm{pyrr}_{2} \mathrm{Py}\right] \mathrm{Ni}\left(\mathrm{NH}_{3}\right)$. Hydrogen atoms except those on ammonia have been omitted for clarity.

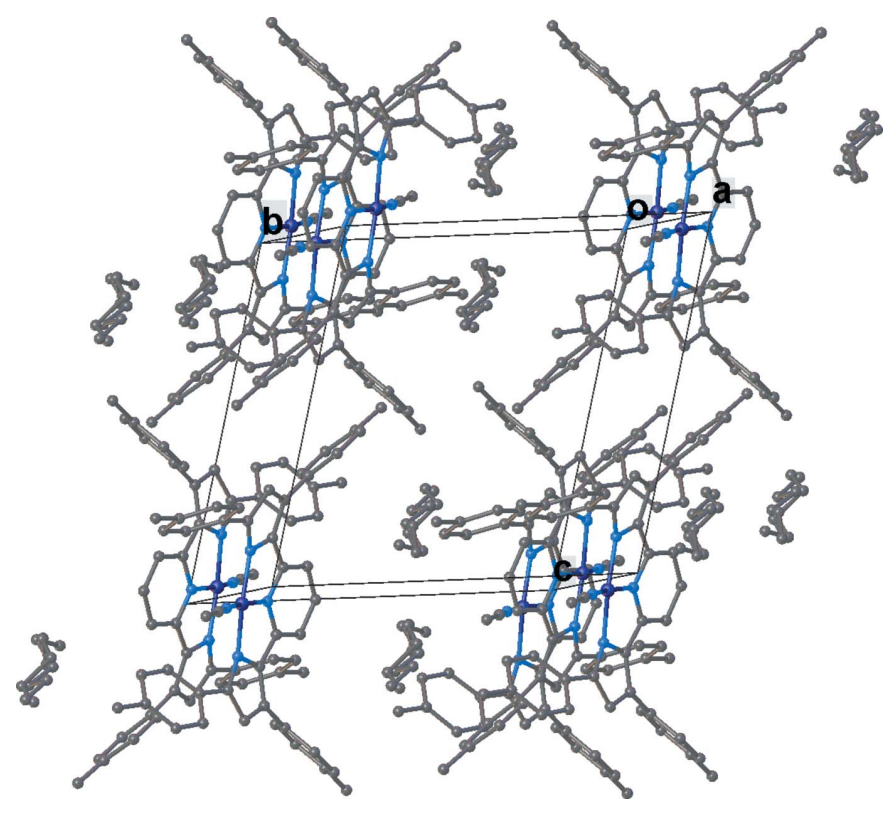

Figure 9

The crystal packing of $\left[\mathrm{pyrr}_{2} \mathrm{Py}\right] \mathrm{Ni}(\mathrm{NCMe})$. Hydrogen atoms have been omitted for clarity.

revealed 38 hits involving ligands with different alkyl or aryl substituents (CSD Version 5.41, Update 2, May 2020; Groom et al., 2016). No nickel pyridine dipyrrolide complexes have been reported thus far. Perhaps the most closely related compounds are the four-coordinate platinum (VIWSIF; Komine et al., 2014), palladium (XIKKIO, XIKKOU; Yadav et al., 2018) and zinc (VIWSIF; Komine et al., 2014) complexes featuring all-nitrogen coordination spheres at the metal. In addition, there are ten hits for related free ligands. Most of them, however, are different solvates of the same ligand system.

\section{Synthesis and crystallization}

All experiments were done under a purified nitrogen atmosphere with standard Schlenk techniques. Solvents were purchased from commercial sources and purified using an Innovative Technology SPS-400 PureSolv solvent-drying system or distilled over conventional drying agents and degassed by the freeze-pump-thaw method three times prior to use. All other chemicals needed were obtained from commercial vendors. Glassware was oven dried at $150^{\circ} \mathrm{C}$ overnight. The NMR spectra were recorded at $25^{\circ} \mathrm{C}$ on JEOL Eclipse 500 and 300 spectrometers $\left({ }^{1} \mathrm{H}: 500.16 \mathrm{MHz}\right.$ or $300.53 \mathrm{MHz}$ ). Proton chemical shifts are reported in ppm versus $\mathrm{Me}_{4} \mathrm{Si}$. Infrared spectra were taken on a JASCO FT-IR 410 spectrometer.

Synthesis of 2,6-bis(3,5-ditolyl-2-pyrrolyl)pyridine, $\left[\operatorname{pyrr}_{2} \mathbf{P y}\right] \mathbf{H}_{2}$ :

1,3-Bis(4-tolyl)-2-propen-1-one (chalcone) was prepared following a literature procedure (Yang et al., 2005) from tolualdehyde and 4-methylacetophenone. Then the chalcone 
$(1.75 \mathrm{~g}, \quad 7.4 \mathrm{mmol})$ was reacted with 2,6-pyridinedicarbaldehyde $(0.50 \mathrm{~g}, 3.7 \mathrm{mmol})$, 3-benzyl-5-(-hydroxyethyl)-4-methylthiazolium chloride $(0.20 \mathrm{~g}, 0.74 \mathrm{mmol})$ and sodium $t$-butoxide $(0.57 \mathrm{~g}, 0.74 \mathrm{mmol})$ in ethanol at reflux for $24 \mathrm{~h}$ to form a brown suspension. Water was added and the mixture was extracted with chloroform. The chloroform was removed to obtain 2,6-bis(2,4-ditolyl-1,4-dioxobutyl)pyridine. This was purified further by rinsing with hexane to get an orange solid. The intermediate ketone was reacted with $\mathrm{NH}_{4} \mathrm{OAc}(2.8 \mathrm{~g}, 37 \mathrm{mmol})$ in ethanol at reflux for $24 \mathrm{~h}$. Water was added and the yellow solid was filtered and washed with water. Then the crude product was suspended in $10 \mathrm{~mL}$ of ethanol and refluxed at $373 \mathrm{~K}$ for $7 \mathrm{~h}$ to obtain 2,6-bis(3,5ditolyl-2-pyrrolyl)pyridine, [pyrr $2 \mathrm{Py} \mathrm{H}_{2}$ as a yellow solid (yield $64 \%) .{ }^{1} \mathrm{H} \mathrm{NMR}\left(\mathrm{CDCl}_{3}, 500.16 \mathrm{MHz}, 298 \mathrm{~K}\right): \delta 2.38(s, 12 \mathrm{H}$, $\left.\mathrm{CH}_{3}\right) 6.57(m, 2 \mathrm{H}), 7.02(d, J=8.05,2 \mathrm{H}), 7.17-7.22(m, 9 \mathrm{H})$, $7.38(d, 4 \mathrm{H}), 7.47(d, J=8 \mathrm{~Hz}, 4 \mathrm{H}), 9.56(2 \mathrm{H}, \mathrm{NH})$.

\section{Synthesis of $\left[\mathrm{pyrr}_{2} \mathrm{Py}\right] \mathrm{Ni}\left(\mathrm{NCCH}_{3}\right)$ :}

A solid sample of the ligand 2,6-bis(3,5-ditolyl-2-pyrrolyl)pyridine ([pyrr $\left.{ }_{2} \mathrm{Py}_{\mathrm{H}} \mathrm{H}_{2} ; 0.10 \mathrm{~g}, 0.175 \mathrm{mmol}\right)$ and $\mathrm{KH}(0.021 \mathrm{~g}$, $0.525 \mathrm{mmol}$ ) were placed in a $50 \mathrm{~mL}$ Schlenk flask. THF ( $c a$ $10 \mathrm{~mL}$ ) was added to the mixture at room temperature and then refluxed for $1.5 \mathrm{~h}$. It was allowed to cool down to room temperature and filtered through a Celite pad, which was then washed with $5 \mathrm{~mL}$ of THF. The filtrate was collected and added to $\mathrm{Ni}(\mathrm{OTf})_{2}(0.062 \mathrm{~g}, 0.175 \mathrm{mmol})$ in $10 \mathrm{~mL}$ of THF and stirred overnight at room temperature. The volatile materials were removed under reduced pressure and the residue was extracted into ether and filtered. Ether was removed under vacuum and $10 \mathrm{~mL}$ of acetonitrile were added. After $1 \mathrm{~h}$, it was filtered, and the filtrate was concentrated to $4 \mathrm{~mL}$. Finally, hexane was layered above the acetonitrile and allowed to diffuse slowly into acetonitrile solution at room temperature, producing brown crystals of $\left[\mathrm{pyrr}_{2} \mathrm{Py}\right] \mathrm{Ni}\left(\mathrm{CH}_{3} \mathrm{CN}\right.$ ) (yield $34 \%) .{ }^{1} \mathrm{H}$ NMR $\left(\mathrm{CDCl}_{3}, 500.16 \mathrm{MHz}, 298 \mathrm{~K}\right): \delta 0.738(s, 3 \mathrm{H}$, $\left.\mathrm{CH}_{3}\right) 2.32\left(s, 6 \mathrm{H}, \mathrm{CH}_{3}\right), 2.37\left(s, 6 \mathrm{H}, \mathrm{CH}_{3}\right) 6.06(s, 2 \mathrm{H}), 6.60(d$, $J=8 \mathrm{~Hz}, 2 \mathrm{H}), 7.04(t, J=8 \mathrm{~Hz}, 1 \mathrm{H}), 7.15(m, 8 \mathrm{H}), 7.36(d, J=$ $8 \mathrm{~Hz}, 4 \mathrm{H}), 7.62(d, J=8.05 \mathrm{~Hz}, 4 \mathrm{H})$.

\section{Synthesis of $\left[\operatorname{pyrr}_{2} \mathbf{P y}\right] \mathbf{N i}(\mathbf{C O})$ :}

A solid sample of the ligand 2,6-bis(3,5-ditolyl-2-pyrrolyl)pyridine $\left(\left[\right.\right.$ pyrr $\left._{2} \mathrm{Py}_{3} \mathrm{H}_{2}\right) \quad(0.10 \mathrm{~g}, 0.175 \mathrm{mmol})$ and $\mathrm{KH}$ $(0.021 \mathrm{~g}, 0.525 \mathrm{mmol})$ were placed in a $50 \mathrm{~mL}$ Schlenk flask. THF ( $c a 10 \mathrm{~mL}$ ) was added to the mixture at room temperature and then refluxed for $1.5 \mathrm{~h}$. It was allowed to cool down to room temperature and filtered through a Celite pad, which was then washed with $5 \mathrm{~mL}$ of THF. The filtrate was added to $\mathrm{Ni}(\mathrm{OTf})_{2}(0.062 \mathrm{~g}, 0.175 \mathrm{mmol})$ in $10 \mathrm{~mL}$ of THF and stirred overnight at room temperature. Then THF was removed and the residue was extracted into ether. Then anhydrous carbon monoxide gas was passed through the ether solution for 20 minutes at $273 \mathrm{~K}$. After stirring for $1 \mathrm{~h}$, the solution was filtered, and the volume of the solution was decreased to $4 \mathrm{~mL}$. Red crystals of [pyrr $\left.{ }_{2} \mathrm{Py}\right] \mathrm{Ni}(\mathrm{CO})$ were observed after keeping the solution in a $253 \mathrm{~K}$ freezer for $3 \mathrm{~d}$ (yield $24 \%$ ). ${ }^{1} \mathrm{H} \mathrm{NMR}$ $\left(\mathrm{CDCl}_{3}, 500.16 \mathrm{MHz}, 298 \mathrm{~K}\right): \delta 2.37\left(s, 6 \mathrm{H}, \mathrm{CH}_{3}\right), 2.38(s, 6 \mathrm{H}$, $\left.\mathrm{CH}_{3}\right) 6.21(s, 2 \mathrm{H}), 6.77(d, J=7.45 \mathrm{~Hz}, 2 \mathrm{H}), 7.02(t, J=8 \mathrm{~Hz}$, $1 \mathrm{H}), 7.21(m, 8 \mathrm{H}), 7.38(d, J=7.5 \mathrm{~Hz}, 4 \mathrm{H}), 7.47(d, J=8.05 \mathrm{~Hz}$,
4H). $\left.{ }^{13} \mathrm{C}_{\{}{ }^{1} \mathrm{H}\right\}$ NMR $\left(\mathrm{CDCl}_{3}, 125.77 \mathrm{MHz}, 298 \mathrm{~K}\right.$, selected): $\delta$ 174.4 (CO). IR (crystals, ATR, selected band) $\mathrm{cm}^{-1}: 2101$ (CO).

\section{Synthesis of $\left[\operatorname{pyrr}_{2} \mathrm{Py}\right] \mathrm{Ni}\left(\mathrm{NH}_{3}\right)$ :}

A solid sample of the ligand 2,6-bis(3,5-ditolyl-2-pyrrolyl)pyridine $\left(\left[\mathrm{pyrr}_{2} \mathrm{Py}\right] \mathrm{H}_{2}\right) \quad(0.10 \mathrm{~g}, 0.175 \mathrm{mmol})$ and $\mathrm{KH}$ $(0.021 \mathrm{~g}, 0.525 \mathrm{mmol})$ were placed in a $50 \mathrm{~mL}$ Schlenk flask. THF ( $c a 10 \mathrm{~mL}$ ) was added to the mixture at room temperature and then refluxed for $1.5 \mathrm{~h}$. It was allowed to cool down to room temperature and filtered through a Celite pad, which was then washed with $5 \mathrm{~mL}$ of THF. The filtrate was added to $\mathrm{Ni}(\mathrm{OTf})_{2}(0.062 \mathrm{~g}, 0.175 \mathrm{mmol})$ in $10 \mathrm{~mL}$ of THF and stirred overnight at room temperature. Then THF was removed and the residue was extracted into ether. Then anhydrous ammonia gas was passed through the ether solution for 20 minutes at $273 \mathrm{~K}$. After stirring for $1 \mathrm{~h}$, the solution was filtered, and the volume of the solution was decreased to $4 \mathrm{~mL}$. Red crystals of $\left[\mathrm{pyrr}_{2} \mathrm{Py}\right] \mathrm{Ni}\left(\mathrm{NH}_{3}\right)$ were formed after keeping the solution in a $253 \mathrm{~K}$ freezer for $3 \mathrm{~d}$ (yield $54 \%$ ). ${ }^{1} \mathrm{H} \mathrm{NMR}$ $\left(\mathrm{CDCl}_{3}, 500.16 \mathrm{MHz}, 298 \mathrm{~K}\right): \delta 0.49\left(s, 3 \mathrm{H}, \mathrm{NH}_{3}\right) 2.35(s, 6 \mathrm{H}$, $\left.\mathrm{CH}_{3}\right), 2.38\left(s, 6 \mathrm{H}, \mathrm{CH}_{3}\right) 6.08(s, 2 \mathrm{H}), 6.63(d, J=8 \mathrm{~Hz}, 2 \mathrm{H}), 7.05$ $(t, J=8.05 \mathrm{~Hz}, 1 \mathrm{H}), 7.19(m, 8 \mathrm{H}), 7.36(d, J=8.05,4 \mathrm{H}), 7.62(d$, $J=7.45 \mathrm{~Hz}, 4 \mathrm{H})$. IR (crystals, ATR, selected bands) $\mathrm{cm}^{-1}$ : 3310, $3360(\mathrm{NH})$.

\section{Refinement}

Crystal data, data collection and structure refinement details for $\left[\operatorname{pyrr}_{2} \mathrm{Py}_{2} \mathrm{H}_{2}, \quad\left[\operatorname{pyrr}_{2} \mathrm{Py}\right] \mathrm{Ni}(\mathrm{CO}), \quad\left[\operatorname{pyrr}_{2} \mathrm{Py}\right] \mathrm{Ni}\left(\mathrm{NH}_{3}\right)\right.$ and $\left[\right.$ pyrr $\left._{2} \mathrm{Py}\right] \mathrm{Ni}(\mathrm{NCMe}) \cdot$ hexane are summarized in Table 1 . Non$\mathrm{H}$ atoms were refined with anisotropic displacement parameters. Hydrogen atoms, except for the $\mathrm{N}-\mathrm{H}$ hydrogen atoms, were placed in calculated positions using riding models, and refined riding on their parent atoms with $\mathrm{C}-\mathrm{H}=0.95 \AA$ and $U_{\text {iso }}(\mathrm{H})=1.2 U_{\text {eq }}(\mathrm{C})$ for aromatic hydrogen atoms, $\mathrm{C}-\mathrm{H}=$ $0.99 \AA$ and $U_{\text {iso }}(\mathrm{H})=1.2 U_{\text {eq }}(\mathrm{C})$ for methylene hydrogen atoms (of hexane), and $\mathrm{C}-\mathrm{H}=0.98 \AA$ with $U_{\text {iso }}(\mathrm{H})=1.5 U_{\text {eq }}(\mathrm{C})$ for methyl hydrogen atoms. The $\mathrm{N}-\mathrm{H}$ hydrogen atoms of $\left[\mathrm{pyrr}_{2} \mathrm{Py}\right] \mathrm{H}_{2}$ and $\left[\mathrm{pyrr}_{2} \mathrm{Py}\right] \mathrm{Ni}\left(\mathrm{NH}_{3}\right)$ were obtained from a difference-Fourier map and refined freely. The nickel carbonyl complex [pyrr $2 \mathrm{Py}] \mathrm{Ni}(\mathrm{CO})$ is located on a plane of symmetry containing the Ni-CO moiety but perpendicular to the [pyrr $2 \mathrm{Py}$ ] ligand plane, and consequently only a half is contained in the asymmetric unit. The complex $\left[\mathrm{pyrr}_{2} \mathrm{Py}\right]-$ $\mathrm{Ni}\left(\mathrm{NCCH}_{3}\right)$ crystallizes with a molecule of hexane, which was disordered over two sites [with refined occupancy rates of $77.9(5) \%$ and $22.1(5) \%]$. C-C bond distances were restrained to a target value of 1.53 (2) $\AA$ (DFIX restraint of SHELXL), 1,3 C...C distances were restrained to be similar to each other (SADI restraint of $S H E L X L$, esd $=0.04 \AA$ ), and $U^{\mathrm{ij}}$ components of ADPs were restrained to be similar for atoms closer to each other than two $\AA$ (SIMU restraint of SHELXL, esd $=0.02 \AA^{2}$ for terminal atoms and $0.01 \AA^{2}$ for all others). 
Table 1

Experimental details.

\begin{tabular}{|c|c|c|c|c|}
\hline & {$\left[\mathrm{pyrr}_{2} \mathrm{PyH}_{2}\right]$} & {$\left[\mathrm{pyrr}_{2} \mathrm{Py}\right] \mathrm{Ni}(\mathrm{CO})$} & {$\left[\operatorname{pyrr}_{2} \mathrm{Py}\right] \mathrm{Ni}\left(\mathrm{NH}_{3}\right)$} & [pyrr 2 Py]Ni(NCMe) \\
\hline \multicolumn{5}{|l|}{ Crystal data } \\
\hline Chemical formula & $\mathrm{C}_{41} \mathrm{H}_{35} \mathrm{~N}_{3}$ & {$\left[\mathrm{Ni}\left(\mathrm{C}_{41} \mathrm{H}_{33} \mathrm{~N}_{3}\right)(\mathrm{CO})\right]$} & {$\left[\mathrm{Ni}\left(\mathrm{C}_{41} \mathrm{H}_{33} \mathrm{~N}_{3}\right)\left(\mathrm{NH}_{3}\right)\right]$} & {$\left[\mathrm{Ni}\left(\mathrm{C}_{41} \mathrm{H}_{33} \mathrm{~N}_{3}\right)\left(\mathrm{C}_{2} \mathrm{H}_{3} \mathrm{~N}\right)\right]$} \\
\hline$M_{\mathrm{r}}$ & 569.72 & 654.42 & 643.45 & 753.64 \\
\hline Temperature $(\mathrm{K})$ & 100 & 100 & 100 & 100 \\
\hline$a, b, c(\AA)$ & $\begin{array}{l}14.8940(15), 35.155(4), \\
\quad 5.9513(6)\end{array}$ & $\begin{array}{l}6.6482(4), 27.1709(18), \\
\quad 9.1322(6)\end{array}$ & $\begin{array}{l}15.9773(6), 14.9441(5), \\
14.3238(5)\end{array}$ & $\begin{array}{l}11.2735(16), 14.1802(19), \\
14.688(2)\end{array}$ \\
\hline$\alpha, \beta, \gamma\left(^{\circ}\right)$ & $90,100.987(2), 90$ & 90, $101.0700(12), 90$ & $90,107.8140(8), 90$ & $\begin{array}{l}67.162(2), 68.881(2) \\
80.665(2)\end{array}$ \\
\hline$Z$ & 4 & 2 & 4 & 2 \\
\hline Radiation type & Mo $K \alpha$ & Mo $K \alpha$ & Mo $K \alpha$ & Мо $K \alpha$ \\
\hline$\mu\left(\mathrm{mm}^{-1}\right)$ & 0.07 & 0.64 & 0.63 & 0.52 \\
\hline Crystal size $(\mathrm{mm})$ & $0.28 \times 0.18 \times 0.12$ & $0.36 \times 0.27 \times 0.05$ & $0.46 \times 0.41 \times 0.14$ & $0.20 \times 0.12 \times 0.09$ \\
\hline \multicolumn{5}{|l|}{ Data collection } \\
\hline $\begin{array}{l}\text { No. of measured, independent } \\
\text { and observed }[I>2 \sigma(I)] \\
\text { reflections }\end{array}$ & $31843,7596,5005$ & $18888,4080,3280$ & $48278,9931,8502$ & $21869,9994,7327$ \\
\hline$R_{\text {int }}$ & 0.076 & 0.057 & 0.026 & 0.058 \\
\hline$(\sin \theta / \lambda)_{\max }\left(\AA^{-1}\right)$ & 0.669 & 0.667 & 0.714 & 0.667 \\
\hline \multicolumn{5}{|l|}{ Refinement } \\
\hline$R\left[F^{2}>2 \sigma\left(F^{2}\right)\right], w R\left(F^{2}\right), S$ & $0.076,0.182,1.06$ & $0.050,0.119,1.07$ & $0.036,0.098,1.05$ & $0.068,0.193,1.00$ \\
\hline No. of reflections & 7596 & 4080 & 9931 & 9994 \\
\hline No. of parameters & 409 & 222 & 431 & 551 \\
\hline No. of restraints & 0 & 0 & 0 & 178 \\
\hline $\mathrm{H}$-atom treatment & $\begin{array}{l}\mathrm{H} \text { atoms treated by a mixture } \\
\text { of independent and } \\
\text { constrained refinement }\end{array}$ & $\begin{array}{l}\text { H-atom parameters } \\
\text { constrained }\end{array}$ & $\begin{array}{l}\mathrm{H} \text { atoms treated by a mixture } \\
\text { of independent and } \\
\text { constrained refinement }\end{array}$ & $\begin{array}{l}\text { H-atom parameters } \\
\text { constrained }\end{array}$ \\
\hline
\end{tabular}

Computer programs: APEX3 and SAINT (Bruker, 2016), SHELXT (Sheldrick, 2015b), SHELXL (Sheldrick, 2015a) and OLEX2 (Dolomanov et al., 2009).

\section{Acknowledgements}

We thank Dr Muhammed Yousufuddin for the collection of data for some of the molecules presented in the manuscript.

\section{Funding information}

Funding for this research was provided by the Welch Foundation (grant No. Y-1289).

\section{References}

Abubekerov, M., Eymann, L. Y. M., Gianetti, T. L. \& Arnold, J. (2016). Dalton Trans. 45, 14581-14590.

Adiraju, V. A. K., Jin, W., Yousufuddin, M. \& Dias, H. V. R. (2020). Z. Anorg. Allg. Chem. 646, 215-219.

Albrecht, M. \& van Koten, G. (2001). Angew. Chem. Int. Ed. 40, 3750-3781.

Alig, L., Fritz, M. \& Schneider, S. (2019). Chem. Rev. 119, 26812751.

Bruker (2016). APEX2, SAINT and SADABS. Bruker AXS Inc. Madison, Wisconsin, USA.

Dias, H. V. R., Huai, L., Jin, W. \& Bott, S. G. (1995). Inorg. Chem. 34, 1973-1974.

Dias, H. V. R., Jin, W., Kim, H.-J. \& Lu, H.-L. (1996). Inorg. Chem. 35 , 2317-2328.

Dias, H. V. R. \& Lovely, C. J. (2008). Chem. Rev. 108, 3223-3238.
Dias, H. V. R. \& Lu, H.-L. (1995). Inorg. Chem. 34, 5380-5382.

Dolomanov, O. V., Bourhis, L. J., Gildea, R. J., Howard, J. A. K. \& Puschmann, H. (2009). J. Appl. Cryst. 42, 339-341.

Gowda, A. S., Petersen, J. L. \& Milsmann, C. (2018). Inorg. Chem. 57, 1919-1934.

Grant, L. N., Carroll, M. E., Carroll, P. J. \& Mindiola, D. J. (2016). Inorg. Chem. 55, 7997-8002.

Groom, C. R., Bruno, I. J., Lightfoot, M. P. \& Ward, S. C. (2016). Acta Cryst. B72, 171-179.

Hakey, B. M., Darmon, J. M., Zhang, Y., Petersen, J. L. \& Milsmann, C. (2019). Inorg. Chem. 58, 1252-1266.

Komine, N., Buell, R. W., Chen, C.-H., Hui, A. K., Pink, M. \& Caulton, K. G. (2014). Inorg. Chem. 53, 1361-1369.

Moulton, C. J. \& Shaw, B. L. (1976). J. Chem. Soc. Dalton Trans. pp. $1020-1024$.

Peris, E. \& Crabtree, R. H. (2004). Coord. Chem. Rev. 248, 22392246.

Peris, E. \& Crabtree, R. H. (2018). Chem. Soc. Rev. 47, 19591968.

Piccirilli, L., Pinheiro, D. L. J. \& Nielsen, M. (2020). Catalysts 10, 773.

Pramanik, A. (2015). MS thesis, The University of Texas at Arlington, Arlington, Texas, USA.

Pramanik, A., Das, A., Yousufuddin, M. \& Dias, H. V. R. (2014). Abstracts of Papers, 247th ACS National Meeting \& Exposition, Dallas, TX, United States, March 16-20, 2014, INOR-266.

Sheldrick, G. M. (2015a). Acta Cryst. A71, 3-8.

Sheldrick, G. M. (2015b). Acta Cryst. C71, 3-8. 
Sorsche, D., Miehlich, M., Searles, K., Gouget, G., Zolnhofer, E. M., Fortier, S., Chen, C.-H., Gau, M. R., Carroll, P. J., Murray, C. B., Caulton, K. G., Khusniyarov, M. M., Meyer, K. \& Mindiola, D. J. (2020). J. Am. Chem. Soc. 142, 8147-8159.

Tapper, A. E., Billo, E. J. \& Golen, J. A. (1993). Inorg. Chim. Acta, 210, 71-75.

Yadav, S., Singh, A., Rashid, N., Ghotia, M., Roy, T. K., Ingole, P. P., Ray, S., Mobin, S. M. \& Dash, C. (2018). ChemistrySelect 3, 94699475.
Yang, J.-X., Tao, X.-T., Yuan, C. X., Yan, Y. X., Wang, L., Liu, Z., Ren, Y. \& Jiang, M. H. (2005). J. Am. Chem. Soc. 127, 32783279.

Zhang, Y., Lee, T. S., Favale, J. M., Leary, D. C., Petersen, J. L., Scholes, G. D., Castellano, F. N. \& Milsmann, C. (2020). Nat. Chem. 12, 345-352.

Zhang, Y., Petersen, J. L. \& Milsmann, C. (2016). J. Am. Chem. Soc. 138, 13115-13118. 


\section{supporting information}

Acta Cryst. (2020). E76, 1741-1747 [https://doi.org/10.1107/S2056989020013341]

Nickel(II) carbonyl, ammonia, and acetonitrile complexes supported by a

pyridine dipyrrolide pincer ligand

\section{H. V. Rasika Dias and Abhijit Pramanik}

Computing details

For all structures, data collection: APEX3 (Bruker, 2016); cell refinement: SAINT (Bruker, 2016); data reduction: SAINT

Bruker, 2016); program(s) used to solve structure: ShelXT (Sheldrick, 2015b); program(s) used to refine structure:

SHELXL (Sheldrick, 2015a); molecular graphics: OLEX2 (Dolomanov et al., 2009); software used to prepare material for publication: OLEX2 (Dolomanov et al., 2009).

2,6-Bis[3,5-bis(4-methylphenyl)pyrrol-2-yl]pyridine (pyrr2PyH2)

\section{Crystal data}

$\mathrm{C}_{41} \mathrm{H}_{35} \mathrm{~N}_{3}$

$M_{r}=569.72$

Monoclinic, $P 2{ }_{1} / c$

$a=14.8940(15) \AA$

$b=35.155(4) \AA$

$c=5.9513(6) \AA$

$\beta=100.987(2)^{\circ}$

$V=3059.0(5) \AA^{3}$

$Z=4$

\section{Data collection}

Bruker D8 Quest with a Photon 100 CMOS detector diffractometer

Radiation source: sealed tube Curved-graphite monochromator Detector resolution: 8 pixels $\mathrm{mm}^{-1}$ $\varphi$ and $\omega$ scans

Absorption correction: multi-scan

(SADABS; Bruker, 2016)

\section{Refinement}

Refinement on $F^{2}$

Least-squares matrix: full

$R\left[F^{2}>2 \sigma\left(F^{2}\right)\right]=0.076$

$w R\left(F^{2}\right)=0.182$

$S=1.06$

7596 reflections

409 parameters

0 restraints
$F(000)=1208$

$D_{\mathrm{x}}=1.237 \mathrm{Mg} \mathrm{m}^{-3}$

Mo $K \alpha$ radiation, $\lambda=0.71073 \AA$

Cell parameters from 6014 reflections

$\theta=3.0-28.0^{\circ}$

$\mu=0.07 \mathrm{~mm}^{-1}$

$T=100 \mathrm{~K}$

Plates, yellow

$0.28 \times 0.18 \times 0.12 \mathrm{~mm}$

$T_{\min }=0.341, T_{\max }=0.431$

31843 measured reflections

7596 independent reflections

5005 reflections with $I>2 \sigma(I)$

$R_{\text {int }}=0.076$

$\theta_{\text {max }}=28.4^{\circ}, \theta_{\min }=2.9^{\circ}$

$h=-19 \rightarrow 19$

$k=-46 \rightarrow 46$

$l=-7 \rightarrow 7$

Primary atom site location: dual

Secondary atom site location: difference Fourier map

Hydrogen site location: mixed

$\mathrm{H}$ atoms treated by a mixture of independent and constrained refinement

$w=1 /\left[\sigma^{2}\left(F_{\mathrm{o}}^{2}\right)+(0.0644 P)^{2}+2.7619 P\right]$ where $P=\left(F_{\mathrm{o}}^{2}+2 F_{\mathrm{c}}{ }^{2}\right) / 3$ 
$(\Delta / \sigma)_{\max }<0.001$

$\Delta \rho_{\max }=0.40 \mathrm{e} \AA^{-3}$

$\Delta \rho_{\min }=-0.25$ e $\AA^{-3}$

Special details

Geometry. All esds (except the esd in the dihedral angle between two 1.s. planes) are estimated using the full covariance matrix. The cell esds are taken into account individually in the estimation of esds in distances, angles and torsion angles; correlations between esds in cell parameters are only used when they are defined by crystal symmetry. An approximate (isotropic) treatment of cell esds is used for estimating esds involving l.s. planes.

Fractional atomic coordinates and isotropic or equivalent isotropic displacement parameters $\left(\hat{A}^{2}\right)$

\begin{tabular}{|c|c|c|c|c|}
\hline & $x$ & $y$ & $z$ & $U_{\text {iso }} * / U_{\text {eq }}$ \\
\hline N1 & $0.31778(13)$ & $0.41078(6)$ & $0.3752(4)$ & $0.0269(5)$ \\
\hline H1 & 0.3085 (17) & $0.3918(8)$ & $0.284(5)$ & $0.023(7)^{*}$ \\
\hline N2 & $0.15143(12)$ & $0.38454(5)$ & 0.2069 & $0.0220(4)$ \\
\hline N3 & $0.15725(13)$ & $0.34057(6)$ & -0.1524 & $0.0218(4)$ \\
\hline $\mathrm{H} 3$ & $0.201(2)$ & $0.3567(8)$ & $-0.090(5)$ & $0.035(8)^{*}$ \\
\hline $\mathrm{C} 1$ & $0.39793(16)$ & $0.42861(7)$ & $0.4672(4)$ & $0.0284(6)$ \\
\hline $\mathrm{C} 2$ & $0.37801(16)$ & $0.45430(8)$ & $0.6255(5)$ & 0.0309 (6) \\
\hline $\mathrm{H} 2$ & 0.420528 & 0.470845 & 0.716611 & $0.037^{*}$ \\
\hline C3 & $0.28267(16)$ & $0.45185(7)$ & $0.6295(4)$ & $0.0268(5)$ \\
\hline $\mathrm{C} 4$ & $0.24695(15)$ & $0.42390(7)$ & $0.4728(4)$ & $0.0239(5)$ \\
\hline $\mathrm{C} 5$ & $0.48235(16)$ & $0.41980(7)$ & $0.3826(5)$ & $0.0298(6)$ \\
\hline C6 & $0.47946(18)$ & $0.40299(9)$ & $0.1714(5)$ & $0.0411(7)$ \\
\hline H6 & 0.421944 & 0.397431 & 0.077399 & $0.049 *$ \\
\hline $\mathrm{C} 7$ & 0.55943 (19) & $0.39409(9)$ & $0.0944(6)$ & $0.0422(7)$ \\
\hline $\mathrm{H} 7$ & 0.555510 & 0.382415 & -0.051101 & $0.051^{*}$ \\
\hline $\mathrm{C} 8$ & $0.64462(17)$ & $0.40191(8)$ & $0.2254(5)$ & $0.0339(6)$ \\
\hline C9 & 0.64744 (19) & $0.41900(10)$ & $0.4340(6)$ & $0.0486(8)$ \\
\hline H9 & 0.705182 & 0.424893 & 0.525887 & $0.058^{*}$ \\
\hline $\mathrm{C} 10$ & $0.56826(18)$ & $0.42799(9)$ & $0.5151(6)$ & $0.0456(8)$ \\
\hline $\mathrm{H} 10$ & 0.572601 & 0.439699 & 0.660598 & $0.055^{*}$ \\
\hline $\mathrm{C} 11$ & $0.73140(18)$ & $0.39198(9)$ & $0.1421(6)$ & $0.0419(7)$ \\
\hline H11A & 0.778705 & 0.410843 & 0.199197 & $0.063^{*}$ \\
\hline H11B & 0.719407 & 0.391943 & -0.025612 & $0.063 *$ \\
\hline $\mathrm{H} 11 \mathrm{C}$ & 0.752380 & 0.366705 & 0.198732 & $0.063 *$ \\
\hline $\mathrm{C} 12$ & $0.23419(16)$ & $0.47692(7)$ & $0.7652(4)$ & $0.0257(5)$ \\
\hline $\mathrm{C} 13$ & $0.27181(17)$ & $0.48578(7)$ & $0.9915(4)$ & $0.0304(6)$ \\
\hline H13 & 0.329417 & 0.475331 & 1.059455 & $0.036^{*}$ \\
\hline $\mathrm{C} 14$ & $0.22680(19)$ & $0.50962(8)$ & $1.1203(5)$ & $0.0327(6)$ \\
\hline H14 & 0.253710 & 0.514926 & 1.274969 & $0.039^{*}$ \\
\hline C15 & $0.14287(18)$ & $0.52581(7)$ & $1.0254(5)$ & $0.0299(6)$ \\
\hline C16 & $0.10546(17)$ & $0.51734(7)$ & $0.7991(5)$ & $0.0286(6)$ \\
\hline H16 & 0.048060 & 0.528006 & 0.731306 & $0.034^{*}$ \\
\hline $\mathrm{C} 17$ & $0.15026(16)$ & $0.49357(7)$ & $0.6694(5)$ & $0.0265(5)$ \\
\hline H17 & 0.123589 & 0.488589 & 0.514138 & $0.032 *$ \\
\hline $\mathrm{C} 18$ & $0.0944(2)$ & $0.55256(8)$ & $1.1610(5)$ & $0.0396(7)$ \\
\hline H18A & 0.106102 & 0.578898 & 1.120927 & $0.059^{*}$ \\
\hline
\end{tabular}




\begin{tabular}{|c|c|c|c|c|}
\hline H18B & 0.117200 & 0.548580 & 1.324950 & $0.059^{*}$ \\
\hline $\mathrm{H} 18 \mathrm{C}$ & 0.028499 & 0.547566 & 1.125210 & $0.059^{*}$ \\
\hline C19 & $0.15686(15)$ & $0.40612(7)$ & 0.3944 (4) & $0.0226(5)$ \\
\hline $\mathrm{C} 20$ & $0.08401(16)$ & $0.40989(7)$ & $0.5100(4)$ & $0.0247(5)$ \\
\hline $\mathrm{H} 20$ & 0.090314 & 0.424150 & 0.647765 & $0.030 *$ \\
\hline $\mathrm{C} 21$ & $0.00241(16)$ & $0.39217(7)$ & $0.4173(4)$ & $0.0250(5)$ \\
\hline $\mathrm{H} 21$ & -0.048992 & 0.394936 & 0.489085 & $0.030 *$ \\
\hline $\mathrm{C} 22$ & $-0.00481(16)$ & $0.37054(7)$ & $0.2219(4)$ & $0.0245(5)$ \\
\hline $\mathrm{H} 22$ & -0.061207 & 0.358902 & 0.155506 & $0.029 *$ \\
\hline $\mathrm{C} 23$ & $0.07227(15)$ & $0.36606(6)$ & $0.1232(4)$ & $0.0205(5)$ \\
\hline $\mathrm{C} 24$ & $0.07687(15)$ & $0.34119(7)$ & $-0.0706(4)$ & $0.0215(5)$ \\
\hline $\mathrm{C} 25$ & $0.01808(15)$ & $0.31582(7)$ & $-0.2046(4)$ & $0.0217(5)$ \\
\hline $\mathrm{C} 26$ & $0.06570(15)$ & $0.30020(7)$ & $-0.3677(4)$ & $0.0235(5)$ \\
\hline H26 & 0.042322 & 0.281964 & -0.481713 & $0.028^{*}$ \\
\hline $\mathrm{C} 27$ & $0.15233(15)$ & $0.31619(7)$ & $-0.3319(4)$ & $0.0219(5)$ \\
\hline $\mathrm{C} 28$ & $-0.07829(15)$ & $0.30748(7)$ & $-0.1857(4)$ & $0.0220(5)$ \\
\hline $\mathrm{C} 29$ & $-0.09980(16)$ & $0.28944(7)$ & 0.0044 (4) & $0.0258(5)$ \\
\hline H29 & -0.051897 & 0.280648 & 0.121771 & $0.031 *$ \\
\hline $\mathrm{C} 30$ & $-0.18999(17)$ & $0.28401(7)$ & $0.0264(4)$ & $0.0279(5)$ \\
\hline H30 & -0.202766 & 0.271797 & 0.159191 & $0.033^{*}$ \\
\hline $\mathrm{C} 31$ & $-0.26155(16)$ & $0.29607(7)$ & $-0.1417(5)$ & $0.0279(6)$ \\
\hline $\mathrm{C} 32$ & $-0.24067(16)$ & $0.31310(7)$ & $-0.3364(5)$ & $0.0300(6)$ \\
\hline H32 & -0.288788 & 0.321051 & -0.455786 & $0.036^{*}$ \\
\hline $\mathrm{C} 33$ & $-0.15012(16)$ & $0.31866(7)$ & $-0.3587(4)$ & $0.0264(5)$ \\
\hline H33 & -0.137268 & 0.330214 & -0.493386 & $0.032 *$ \\
\hline C34 & $-0.35951(18)$ & $0.29081(9)$ & $-0.1141(6)$ & $0.0425(7)$ \\
\hline $\mathrm{H} 34 \mathrm{~A}$ & -0.376282 & 0.311221 & -0.017785 & $0.064 *$ \\
\hline H34B & -0.365963 & 0.266169 & -0.041886 & $0.064 *$ \\
\hline $\mathrm{H} 34 \mathrm{C}$ & -0.399856 & 0.291641 & -0.264779 & $0.064 *$ \\
\hline $\mathrm{C} 35$ & $0.23013(15)$ & $0.31055(7)$ & $-0.4467(4)$ & $0.0224(5)$ \\
\hline C36 & $0.21959(17)$ & $0.29117(7)$ & $-0.6546(4)$ & $0.0258(5)$ \\
\hline H36 & 0.161229 & 0.281478 & -0.722971 & $0.031^{*}$ \\
\hline C37 & $0.29340(17)$ & $0.28587(7)$ & $-0.7625(4)$ & $0.0274(5)$ \\
\hline H37 & 0.284801 & 0.272442 & -0.903541 & $0.033^{*}$ \\
\hline $\mathrm{C} 38$ & $0.38005(16)$ & $0.29991(7)$ & $-0.6680(4)$ & $0.0287(6)$ \\
\hline C39 & $0.39012(16)$ & $0.31946(8)$ & $-0.4625(5)$ & $0.0321(6)$ \\
\hline H39 & 0.448292 & 0.329511 & -0.395562 & $0.039 *$ \\
\hline $\mathrm{C} 40$ & $0.31675(16)$ & $0.32462(8)$ & $-0.3528(5)$ & $0.0295(6)$ \\
\hline $\mathrm{H} 40$ & 0.325632 & 0.337963 & -0.211463 & $0.035^{*}$ \\
\hline C41 & $0.46052(19)$ & $0.29329(9)$ & $-0.7846(5)$ & $0.0396(7)$ \\
\hline $\mathrm{H} 41 \mathrm{~A}$ & 0.505142 & 0.313831 & -0.744108 & $0.059 *$ \\
\hline H41B & 0.439234 & 0.292851 & -0.950850 & $0.059 *$ \\
\hline $\mathrm{H} 41 \mathrm{C}$ & 0.489307 & 0.268885 & -0.734267 & $0.059 *$ \\
\hline
\end{tabular}

Atomic displacement parameters $\left(\AA^{2}\right)$

\begin{tabular}{lllllll}
\hline & $U^{11}$ & $U^{22}$ & $U^{33}$ & $U^{12}$ & $U^{13}$ & $U^{23}$ \\
\hline $\mathrm{N} 1$ & $0.0196(10)$ & $0.0316(12)$ & $0.0297(12)$ & $0.0012(8)$ & $0.0049(8)$ & $-0.0087(10)$
\end{tabular}




\begin{tabular}{|c|c|c|c|c|c|c|}
\hline N2 & $0.0167(9)$ & $0.0257(10)$ & $0.0240(11)$ & $0.0030(8)$ & $0.0051(8)$ & $0.0003(8)$ \\
\hline N3 & $0.0155(9)$ & $0.0277(11)$ & $0.0224(10)$ & $0.0006(8)$ & $0.0041(8)$ & $-0.0005(8)$ \\
\hline $\mathrm{C} 1$ & 0.0197 (12) & $0.0332(14)$ & $0.0315(14)$ & $0.0017(10)$ & $0.0025(10)$ & $-0.0049(11)$ \\
\hline $\mathrm{C} 2$ & $0.0203(12)$ & $0.0380(14)$ & $0.0322(14)$ & $0.0016(10)$ & $-0.0007(10)$ & $-0.0084(12)$ \\
\hline $\mathrm{C} 3$ & $0.0214(11)$ & $0.0311(13)$ & $0.0270(13)$ & $0.0054(10)$ & $0.0023(10)$ & $-0.0012(11)$ \\
\hline $\mathrm{C} 4$ & $0.0199(11)$ & $0.0271(12)$ & $0.0254(13)$ & $0.0047(9)$ & $0.0061(9)$ & $0.0001(10)$ \\
\hline $\mathrm{C} 5$ & $0.0203(12)$ & $0.0307(14)$ & $0.0385(15)$ & $0.0021(10)$ & $0.0062(11)$ & $-0.0067(12)$ \\
\hline C6 & $0.0221(13)$ & $0.0505(18)$ & 0.0509 (19) & $-0.0024(12)$ & $0.0074(12)$ & $-0.0149(15)$ \\
\hline C7 & $0.0290(14)$ & $0.0495(18)$ & 0.0504 (19) & $-0.0028(12)$ & $0.0130(13)$ & $-0.0179(15)$ \\
\hline $\mathrm{C} 8$ & $0.0218(12)$ & $0.0321(14)$ & $0.0497(18)$ & $0.0006(10)$ & $0.0117(12)$ & $-0.0001(13)$ \\
\hline C9 & 0.0199 (13) & $0.060(2)$ & $0.063(2)$ & $-0.0009(13)$ & $0.0015(13)$ & $-0.0137(17)$ \\
\hline $\mathrm{C} 10$ & 0.0257 (14) & $0.060(2)$ & $0.0500(19)$ & $-0.0005(13)$ & $0.0037(13)$ & $-0.0205(16)$ \\
\hline $\mathrm{C} 11$ & $0.0251(14)$ & $0.0444(17)$ & $0.060(2)$ & $-0.0001(12)$ & $0.0179(13)$ & $0.0017(15)$ \\
\hline C12 & $0.0230(12)$ & $0.0261(13)$ & $0.0282(13)$ & $0.0019(9)$ & $0.0056(10)$ & $-0.0037(10)$ \\
\hline $\mathrm{C} 13$ & $0.0276(13)$ & $0.0338(14)$ & $0.0286(14)$ & $0.0050(10)$ & $0.0023(11)$ & $-0.0023(11)$ \\
\hline $\mathrm{C} 14$ & $0.0402(15)$ & $0.0322(14)$ & $0.0260(14)$ & $0.0027(11)$ & $0.0071(11)$ & $-0.0039(11)$ \\
\hline $\mathrm{C} 15$ & $0.0355(14)$ & $0.0259(13)$ & $0.0317(14)$ & $0.0020(10)$ & $0.0147(11)$ & $0.0011(11)$ \\
\hline C16 & $0.0247(12)$ & $0.0254(13)$ & $0.0367(15)$ & $0.0031(10)$ & $0.0087(11)$ & $0.0009(11)$ \\
\hline $\mathrm{C} 17$ & 0.0225 (12) & $0.0258(13)$ & $0.0309(14)$ & $-0.0005(9)$ & $0.0042(10)$ & $-0.0027(10)$ \\
\hline $\mathrm{C} 18$ & 0.0478 (17) & $0.0346(15)$ & $0.0415(17)$ & $0.0094(13)$ & $0.0212(14)$ & $-0.0009(13)$ \\
\hline C19 & $0.0213(11)$ & $0.0215(12)$ & $0.0253(13)$ & $0.0028(9)$ & $0.0052(9)$ & $0.0015(10)$ \\
\hline C20 & $0.0257(12)$ & $0.0241(12)$ & $0.0263(13)$ & $0.0033(9)$ & $0.0098(10)$ & $-0.0005(10)$ \\
\hline $\mathrm{C} 21$ & $0.0226(12)$ & $0.0227(12)$ & $0.0333(14)$ & $0.0036(9)$ & $0.0141(10)$ & $0.0010(10)$ \\
\hline $\mathrm{C} 22$ & $0.0201(11)$ & $0.0255(12)$ & $0.0294(13)$ & 0.0007 (9) & $0.0084(10)$ & $0.0020(10)$ \\
\hline $\mathrm{C} 23$ & $0.0184(11)$ & $0.0225(11)$ & $0.0213(12)$ & $0.0020(9)$ & $0.0054(9)$ & $0.0039(9)$ \\
\hline C24 & $0.0171(10)$ & $0.0271(12)$ & $0.0212(12)$ & $0.0026(9)$ & $0.0061(9)$ & $0.0018(10)$ \\
\hline $\mathrm{C} 25$ & $0.0185(11)$ & $0.0248(12)$ & $0.0216(12)$ & $0.0003(9)$ & 0.0032 (9) & $0.0028(10)$ \\
\hline $\mathrm{C} 26$ & $0.0203(11)$ & $0.0276(13)$ & $0.0222(12)$ & $0.0000(9)$ & 0.0027 (9) & $0.0013(10)$ \\
\hline $\mathrm{C} 27$ & $0.0176(11)$ & $0.0266(12)$ & $0.0211(12)$ & $0.0023(9)$ & $0.0029(9)$ & $0.0024(10)$ \\
\hline $\mathrm{C} 28$ & $0.0191(11)$ & $0.0225(12)$ & $0.0248(12)$ & $-0.0005(9)$ & $0.0053(9)$ & $-0.0024(9)$ \\
\hline $\mathrm{C} 29$ & $0.0228(12)$ & $0.0309(13)$ & $0.0234(13)$ & $-0.0007(10)$ & $0.0037(10)$ & $0.0013(10)$ \\
\hline $\mathrm{C} 30$ & $0.0306(13)$ & $0.0297(13)$ & $0.0259(13)$ & $-0.0060(10)$ & $0.0120(11)$ & $-0.0011(10)$ \\
\hline C31 & $0.0202(12)$ & $0.0278(13)$ & $0.0381(15)$ & $-0.0033(9)$ & $0.0114(10)$ & $-0.0069(11)$ \\
\hline $\mathrm{C} 32$ & $0.0201(12)$ & $0.0331(14)$ & $0.0360(15)$ & $0.0014(10)$ & $0.0034(10)$ & $0.0022(11)$ \\
\hline $\mathrm{C} 33$ & $0.0202(11)$ & $0.0322(13)$ & $0.0276(13)$ & $-0.0003(10)$ & $0.0071(10)$ & $0.0039(11)$ \\
\hline $\mathrm{C} 34$ & $0.0243(14)$ & $0.0534(18)$ & $0.0538(19)$ & $-0.0074(12)$ & $0.0179(13)$ & $-0.0046(15)$ \\
\hline C35 & $0.0195(11)$ & $0.0249(12)$ & $0.0231(12)$ & $0.0058(9)$ & $0.0048(9)$ & $0.0038(10)$ \\
\hline C36 & $0.0242(12)$ & $0.0270(13)$ & $0.0258(13)$ & $0.0018(9)$ & $0.0035(10)$ & $0.0027(10)$ \\
\hline C37 & $0.0302(13)$ & $0.0299(13)$ & $0.0234(13)$ & $0.0052(10)$ & $0.0082(10)$ & $-0.0003(10)$ \\
\hline $\mathrm{C} 38$ & $0.0221(12)$ & $0.0351(14)$ & $0.0311(14)$ & $0.0080(10)$ & $0.0110(10)$ & $0.0046(11)$ \\
\hline C39 & $0.0160(11)$ & $0.0453(16)$ & $0.0359(15)$ & $0.0023(10)$ & $0.0071(10)$ & $-0.0037(12)$ \\
\hline C40 & $0.0222(12)$ & $0.0386(15)$ & $0.0282(14)$ & $0.0013(10)$ & $0.0058(10)$ & -0.0058 \\
\hline C41 & $0.0289(14)$ & $0.0569(19)$ & $0.0368(16)$ & $0.0090(13)$ & $0.0158(12)$ & $0.0023(14)$ \\
\hline
\end{tabular}

Geometric parameters $\left(\AA,{ }^{\circ}\right)$

\begin{tabular}{llll}
\hline $\mathrm{N} 1-\mathrm{H} 1$ & $0.86(3)$ & $\mathrm{C} 19-\mathrm{C} 20$ & $1.398(3)$ \\
$\mathrm{N} 1-\mathrm{C} 1$ & $1.367(3)$ & $\mathrm{C} 20-\mathrm{H} 20$ & 0.9500
\end{tabular}




\begin{tabular}{|c|c|c|c|}
\hline $\mathrm{N} 1-\mathrm{C} 4$ & $1.377(3)$ & $\mathrm{C} 20-\mathrm{C} 21$ & $1.384(3)$ \\
\hline $\mathrm{N} 2-\mathrm{C} 19$ & $1.339(3)$ & $\mathrm{C} 21-\mathrm{H} 21$ & 0.9500 \\
\hline $\mathrm{N} 2-\mathrm{C} 23$ & $1.355(3)$ & $\mathrm{C} 21-\mathrm{C} 22$ & $1.377(3)$ \\
\hline N3-H3 & $0.89(3)$ & $\mathrm{C} 22-\mathrm{H} 22$ & 0.9500 \\
\hline N3-C24 & $1.376(3)$ & $\mathrm{C} 22-\mathrm{C} 23$ & $1.394(3)$ \\
\hline $\mathrm{N} 3-\mathrm{C} 27$ & $1.360(3)$ & $\mathrm{C} 23-\mathrm{C} 24$ & $1.460(3)$ \\
\hline $\mathrm{C} 1-\mathrm{C} 2$ & $1.378(4)$ & $\mathrm{C} 24-\mathrm{C} 25$ & $1.390(3)$ \\
\hline $\mathrm{C} 1-\mathrm{C} 5$ & $1.474(3)$ & $\mathrm{C} 25-\mathrm{C} 26$ & $1.417(3)$ \\
\hline $\mathrm{C} 2-\mathrm{H} 2$ & 0.9500 & $\mathrm{C} 25-\mathrm{C} 28$ & $1.490(3)$ \\
\hline $\mathrm{C} 2-\mathrm{C} 3$ & $1.427(3)$ & $\mathrm{C} 26-\mathrm{H} 26$ & 0.9500 \\
\hline $\mathrm{C} 3-\mathrm{C} 4$ & $1.389(3)$ & $\mathrm{C} 26-\mathrm{C} 27$ & $1.386(3)$ \\
\hline $\mathrm{C} 3-\mathrm{C} 12$ & $1.474(3)$ & $\mathrm{C} 27-\mathrm{C} 35$ & $1.466(3)$ \\
\hline $\mathrm{C} 4-\mathrm{C} 19$ & $1.473(3)$ & $\mathrm{C} 28-\mathrm{C} 29$ & $1.387(3)$ \\
\hline $\mathrm{C} 5-\mathrm{C} 6$ & $1.382(4)$ & $\mathrm{C} 28-\mathrm{C} 33$ & $1.393(3)$ \\
\hline $\mathrm{C} 5-\mathrm{C} 10$ & $1.399(4)$ & $\mathrm{C} 29-\mathrm{H} 29$ & 0.9500 \\
\hline C6-H6 & 0.9500 & $\mathrm{C} 29-\mathrm{C} 30$ & $1.387(3)$ \\
\hline $\mathrm{C} 6-\mathrm{C} 7$ & $1.391(4)$ & $\mathrm{C} 30-\mathrm{H} 30$ & 0.9500 \\
\hline $\mathrm{C} 7-\mathrm{H} 7$ & 0.9500 & $\mathrm{C} 30-\mathrm{C} 31$ & $1.382(4)$ \\
\hline $\mathrm{C} 7-\mathrm{C} 8$ & $1.384(4)$ & $\mathrm{C} 31-\mathrm{C} 32$ & $1.391(4)$ \\
\hline $\mathrm{C} 8-\mathrm{C} 9$ & $1.372(4)$ & C31-C34 & $1.510(3)$ \\
\hline $\mathrm{C} 8-\mathrm{C} 11$ & $1.511(4)$ & C $32-\mathrm{H} 32$ & 0.9500 \\
\hline C9- & 0.9500 & $\mathrm{C} 32-\mathrm{C} 33$ & 1.394 (3) \\
\hline $\mathrm{C} 9-\mathrm{C} 10$ & $1.393(4)$ & C33-H33 & 0.9500 \\
\hline $\mathrm{C} 10-\mathrm{H} 10$ & 0.9500 & $\mathrm{C} 34-\mathrm{H} 34 \mathrm{~A}$ & 0.9800 \\
\hline $\mathrm{C} 11-\mathrm{H} 11 \mathrm{~A}$ & 0.9800 & C34-H34B & 0.9800 \\
\hline C11-H11B & 0.9800 & $\mathrm{C} 34-\mathrm{H} 34 \mathrm{C}$ & 0.9800 \\
\hline $\mathrm{C} 11-\mathrm{H} 11 \mathrm{C}$ & 0.9800 & $\mathrm{C} 35-\mathrm{C} 36$ & $1.395(3)$ \\
\hline $\mathrm{C} 12-\mathrm{C} 13$ & $1.392(4)$ & $\mathrm{C} 35-\mathrm{C} 40$ & $1.395(3)$ \\
\hline $\mathrm{C} 12-\mathrm{C} 17$ & $1.400(3)$ & C36-H36 & 0.9500 \\
\hline C13-H13 & 0.9500 & $\mathrm{C} 36-\mathrm{C} 37$ & $1.388(3)$ \\
\hline $\mathrm{C} 13-\mathrm{C} 14$ & $1.391(4)$ & C37-H37 & 0.9500 \\
\hline C14-H14 & 0.9500 & $\mathrm{C} 37-\mathrm{C} 38$ & $1.396(4)$ \\
\hline $\mathrm{C} 14-\mathrm{C} 15$ & $1.391(4)$ & $\mathrm{C} 38-\mathrm{C} 39$ & $1.386(4)$ \\
\hline $\mathrm{C} 15-\mathrm{C} 16$ & $1.388(4)$ & $\mathrm{C} 38-\mathrm{C} 41$ & $1.513(3)$ \\
\hline $\mathrm{C} 15-\mathrm{C} 18$ & $1.510(4)$ & C39-H39 & 0.9500 \\
\hline $\mathrm{C} 16-\mathrm{H} 16$ & 0.9500 & $\mathrm{C} 39-\mathrm{C} 40$ & $1.388(3)$ \\
\hline $\mathrm{C} 16-\mathrm{C} 17$ & $1.391(3)$ & $\mathrm{C} 40-\mathrm{H} 40$ & 0.9500 \\
\hline C17-H17 & 0.9500 & $\mathrm{C} 41-\mathrm{H} 41 \mathrm{~A}$ & 0.9800 \\
\hline C18-H18A & 0.9800 & $\mathrm{C} 41-\mathrm{H} 41 \mathrm{~B}$ & 0.9800 \\
\hline C18-H18B & 0.9800 & $\mathrm{C} 41-\mathrm{H} 41 \mathrm{C}$ & 0.9800 \\
\hline $\mathrm{C} 18-\mathrm{H} 18 \mathrm{C}$ & 0.9800 & & \\
\hline $\mathrm{C} 1-\mathrm{N} 1-\mathrm{H} 1$ & 129.5 (17) & $\mathrm{C} 21-\mathrm{C} 20-\mathrm{C} 19$ & $117.8(2)$ \\
\hline $\mathrm{C} 1-\mathrm{N} 1-\mathrm{C} 4$ & $111.0(2)$ & $\mathrm{C} 21-\mathrm{C} 20-\mathrm{H} 20$ & 121.1 \\
\hline $\mathrm{C} 4-\mathrm{N} 1-\mathrm{H} 1$ & $119.0(17)$ & $\mathrm{C} 20-\mathrm{C} 21-\mathrm{H} 21$ & 119.8 \\
\hline $\mathrm{C} 19-\mathrm{N} 2-\mathrm{C} 23$ & $119.21(19)$ & $\mathrm{C} 22-\mathrm{C} 21-\mathrm{C} 20$ & $120.5(2)$ \\
\hline $\mathrm{C} 24-\mathrm{N} 3-\mathrm{H} 3$ & $117.5(18)$ & $\mathrm{C} 22-\mathrm{C} 21-\mathrm{H} 21$ & 119.8 \\
\hline $\mathrm{C} 27-\mathrm{N} 3-\mathrm{H} 3$ & $130.8(18)$ & $\mathrm{C} 21-\mathrm{C} 22-\mathrm{H} 22$ & 120.7 \\
\hline
\end{tabular}




\begin{tabular}{|c|c|}
\hline $\mathrm{C} 27-\mathrm{N} 3-\mathrm{C} 24$ & $111.56(19$ \\
\hline $\mathrm{N} 1-\mathrm{C} 1-\mathrm{C} 2$ & $106.8(2)$ \\
\hline $\mathrm{N} 1-\mathrm{C} 1-\mathrm{C} 5$ & $120.2(2)$ \\
\hline $\mathrm{C} 2-\mathrm{C} 1-\mathrm{C} 5$ & $132.9(2)$ \\
\hline $\mathrm{C} 1-\mathrm{C} 2-\mathrm{H} 2$ & 125.8 \\
\hline $\mathrm{C} 1-\mathrm{C} 2-\mathrm{C} 3$ & $108.5(2)$ \\
\hline $\mathrm{C} 3-\mathrm{C} 2-\mathrm{H} 2$ & 125.8 \\
\hline $\mathrm{C} 2-\mathrm{C} 3-\mathrm{C} 12$ & $124.5(2)$ \\
\hline $\mathrm{C} 4-\mathrm{C} 3-\mathrm{C} 2$ & $106.6(2)$ \\
\hline $\mathrm{C} 4-\mathrm{C} 3-\mathrm{C} 12$ & $128.7(2)$ \\
\hline $\mathrm{N} 1-\mathrm{C} 4-\mathrm{C} 3$ & $107.1(2)$ \\
\hline $\mathrm{N} 1-\mathrm{C} 4-\mathrm{C} 19$ & $116.7(2)$ \\
\hline $\mathrm{C} 3-\mathrm{C} 4-\mathrm{C} 19$ & $136.2(2)$ \\
\hline $\mathrm{C} 6-\mathrm{C} 5-\mathrm{C} 1$ & $121.3(2)$ \\
\hline $\mathrm{C} 6-\mathrm{C} 5-\mathrm{C} 10$ & $117.9(2)$ \\
\hline $\mathrm{C} 10-\mathrm{C} 5-\mathrm{C} 1$ & $120.8(2)$ \\
\hline $\mathrm{C} 5-\mathrm{C} 6-\mathrm{H} 6$ & 119.5 \\
\hline $\mathrm{C} 5-\mathrm{C} 6-\mathrm{C} 7$ & $121.0(3)$ \\
\hline $\mathrm{C} 7-\mathrm{C} 6-\mathrm{H} 6$ & 119.5 \\
\hline $\mathrm{C} 6-\mathrm{C} 7-\mathrm{H} 7$ & 119.3 \\
\hline $\mathrm{C} 8-\mathrm{C} 7-\mathrm{C} 6$ & $121.3(3)$ \\
\hline $\mathrm{C} 8-\mathrm{C} 7-\mathrm{H} 7$ & 119.3 \\
\hline $\mathrm{C} 7-\mathrm{C} 8-\mathrm{C} 11$ & $121.3(3)$ \\
\hline $\mathrm{C} 9-\mathrm{C} 8-\mathrm{C} 7$ & $117.6(2)$ \\
\hline $\mathrm{C} 9-\mathrm{C} 8-\mathrm{C} 11$ & $121.2(3)$ \\
\hline $\mathrm{C} 8-\mathrm{C} 9-\mathrm{H} 9$ & 119.0 \\
\hline $\mathrm{C} 8-\mathrm{C} 9-\mathrm{C} 10$ & $122.0(3)$ \\
\hline $\mathrm{C} 10-\mathrm{C} 9-\mathrm{H} 9$ & 119.0 \\
\hline $\mathrm{C} 5-\mathrm{C} 10-\mathrm{H} 10$ & 119.9 \\
\hline $\mathrm{C} 9-\mathrm{C} 10-\mathrm{C} 5$ & $120.1(3)$ \\
\hline $\mathrm{C} 9-\mathrm{C} 10-\mathrm{H} 10$ & 119.9 \\
\hline $\mathrm{C} 8-\mathrm{C} 11-\mathrm{H} 11 \mathrm{~A}$ & 109.5 \\
\hline $\mathrm{C} 8-\mathrm{C} 11-\mathrm{H} 11 \mathrm{~B}$ & 109.5 \\
\hline $\mathrm{C} 8-\mathrm{C} 11-\mathrm{H} 11 \mathrm{C}$ & 109.5 \\
\hline $\mathrm{H} 11 \mathrm{~A}-\mathrm{C} 11-\mathrm{H} 11 \mathrm{~B}$ & 109.5 \\
\hline $\mathrm{H} 11 \mathrm{~A}-\mathrm{C} 11-\mathrm{H} 11 \mathrm{C}$ & 109.5 \\
\hline $\mathrm{H} 11 \mathrm{~B}-\mathrm{C} 11-\mathrm{H} 11 \mathrm{C}$ & 109.5 \\
\hline $\mathrm{C} 13-\mathrm{C} 12-\mathrm{C} 3$ & $121.0(2)$ \\
\hline $\mathrm{C} 13-\mathrm{C} 12-\mathrm{C} 17$ & $117.7(2)$ \\
\hline $\mathrm{C} 17-\mathrm{C} 12-\mathrm{C} 3$ & $121.3(2)$ \\
\hline $\mathrm{C} 12-\mathrm{C} 13-\mathrm{H} 13$ & 119.3 \\
\hline $\mathrm{C} 14-\mathrm{C} 13-\mathrm{C} 12$ & $121.4(2)$ \\
\hline $\mathrm{C} 14-\mathrm{C} 13-\mathrm{H} 13$ & 119.3 \\
\hline $\mathrm{C} 13-\mathrm{C} 14-\mathrm{H} 14$ & 119.6 \\
\hline $\mathrm{C} 13-\mathrm{C} 14-\mathrm{C} 15$ & $120.9(3)$ \\
\hline $\mathrm{C} 15-\mathrm{C} 14-\mathrm{H} 14$ & 119.6 \\
\hline $\mathrm{C} 14-\mathrm{C} 15-\mathrm{C} 18$ & $121.4(3)$ \\
\hline $\mathrm{C} 16-\mathrm{C} 15-\mathrm{C} 14$ & $118.0(2)$ \\
\hline
\end{tabular}

\begin{tabular}{|c|c|}
\hline $\mathrm{C} 21-\mathrm{C} 22-\mathrm{C} 23$ & $118.7(2)$ \\
\hline $\mathrm{C} 23-\mathrm{C} 22-\mathrm{H} 22$ & 120.7 \\
\hline $\mathrm{N} 2-\mathrm{C} 23-\mathrm{C} 22$ & $121.3(2)$ \\
\hline $\mathrm{N} 2-\mathrm{C} 23-\mathrm{C} 24$ & $114.15(19)$ \\
\hline $\mathrm{C} 22-\mathrm{C} 23-\mathrm{C} 24$ & $124.5(2)$ \\
\hline $\mathrm{N} 3-\mathrm{C} 24-\mathrm{C} 23$ & $117.7(2)$ \\
\hline $\mathrm{N} 3-\mathrm{C} 24-\mathrm{C} 25$ & $106.4(2)$ \\
\hline $\mathrm{C} 25-\mathrm{C} 24-\mathrm{C} 23$ & $135.9(2)$ \\
\hline $\mathrm{C} 24-\mathrm{C} 25-\mathrm{C} 26$ & $107.4(2)$ \\
\hline $\mathrm{C} 24-\mathrm{C} 25-\mathrm{C} 28$ & $126.4(2)$ \\
\hline $\mathrm{C} 26-\mathrm{C} 25-\mathrm{C} 28$ & $126.2(2)$ \\
\hline $\mathrm{C} 25-\mathrm{C} 26-\mathrm{H} 26$ & 126.0 \\
\hline $\mathrm{C} 27-\mathrm{C} 26-\mathrm{C} 25$ & $108.1(2)$ \\
\hline $\mathrm{C} 27-\mathrm{C} 26-\mathrm{H} 26$ & 126.0 \\
\hline $\mathrm{N} 3-\mathrm{C} 27-\mathrm{C} 26$ & $106.6(2)$ \\
\hline N3-C27-C35 & $121.5(2)$ \\
\hline $\mathrm{C} 26-\mathrm{C} 27-\mathrm{C} 35$ & $131.9(2)$ \\
\hline $\mathrm{C} 29-\mathrm{C} 28-\mathrm{C} 25$ & $121.8(2)$ \\
\hline $\mathrm{C} 29-\mathrm{C} 28-\mathrm{C} 33$ & $117.9(2)$ \\
\hline $\mathrm{C} 33-\mathrm{C} 28-\mathrm{C} 25$ & $120.2(2)$ \\
\hline $\mathrm{C} 28-\mathrm{C} 29-\mathrm{H} 29$ & 119.4 \\
\hline $\mathrm{C} 28-\mathrm{C} 29-\mathrm{C} 30$ & $121.2(2)$ \\
\hline $\mathrm{C} 30-\mathrm{C} 29-\mathrm{H} 29$ & 119.4 \\
\hline $\mathrm{C} 29-\mathrm{C} 30-\mathrm{H} 30$ & 119.5 \\
\hline $\mathrm{C} 31-\mathrm{C} 30-\mathrm{C} 29$ & $121.1(2)$ \\
\hline $\mathrm{C} 31-\mathrm{C} 30-\mathrm{H} 30$ & 119.5 \\
\hline $\mathrm{C} 30-\mathrm{C} 31-\mathrm{C} 32$ & $118.1(2)$ \\
\hline $\mathrm{C} 30-\mathrm{C} 31-\mathrm{C} 34$ & $120.8(2)$ \\
\hline $\mathrm{C} 32-\mathrm{C} 31-\mathrm{C} 34$ & $121.1(2)$ \\
\hline $\mathrm{C} 31-\mathrm{C} 32-\mathrm{H} 32$ & 119.5 \\
\hline $\mathrm{C} 31-\mathrm{C} 32-\mathrm{C} 33$ & $120.9(2)$ \\
\hline $\mathrm{C} 33-\mathrm{C} 32-\mathrm{H} 32$ & 119.5 \\
\hline $\mathrm{C} 28-\mathrm{C} 33-\mathrm{C} 32$ & $120.7(2)$ \\
\hline $\mathrm{C} 28-\mathrm{C} 33-\mathrm{H} 33$ & 119.6 \\
\hline C $32-\mathrm{C} 33-\mathrm{H} 33$ & 119.6 \\
\hline $\mathrm{C} 31-\mathrm{C} 34-\mathrm{H} 34 \mathrm{~A}$ & 109.5 \\
\hline C $31-\mathrm{C} 34-\mathrm{H} 34 \mathrm{~B}$ & 109.5 \\
\hline $\mathrm{C} 31-\mathrm{C} 34-\mathrm{H} 34 \mathrm{C}$ & 109.5 \\
\hline $\mathrm{H} 34 \mathrm{~A}-\mathrm{C} 34-\mathrm{H} 34 \mathrm{~B}$ & 109.5 \\
\hline $\mathrm{H} 34 \mathrm{~A}-\mathrm{C} 34-\mathrm{H} 34 \mathrm{C}$ & 109.5 \\
\hline $\mathrm{H} 34 \mathrm{~B}-\mathrm{C} 34-\mathrm{H} 34 \mathrm{C}$ & 109.5 \\
\hline $\mathrm{C} 36-\mathrm{C} 35-\mathrm{C} 27$ & $120.9(2)$ \\
\hline $\mathrm{C} 36-\mathrm{C} 35-\mathrm{C} 40$ & $117.9(2)$ \\
\hline $\mathrm{C} 40-\mathrm{C} 35-\mathrm{C} 27$ & $121.2(2)$ \\
\hline $\mathrm{C} 35-\mathrm{C} 36-\mathrm{H} 36$ & 119.6 \\
\hline $\mathrm{C} 37-\mathrm{C} 36-\mathrm{C} 35$ & $120.7(2)$ \\
\hline C37-C $36-\mathrm{H} 36$ & 119.6 \\
\hline $\mathrm{C} 36-\mathrm{C} 37-\mathrm{H} 37$ & 119.4 \\
\hline
\end{tabular}




\begin{tabular}{|c|c|c|c|}
\hline $\mathrm{C} 16-\mathrm{C} 15-\mathrm{C} 18$ & $120.5(2)$ & $\mathrm{C} 36-\mathrm{C} 37-\mathrm{C} 38$ & $121.3(2)$ \\
\hline $\mathrm{C} 15-\mathrm{C} 16-\mathrm{H} 16$ & 119.3 & $\mathrm{C} 38-\mathrm{C} 37-\mathrm{H} 37$ & 119.4 \\
\hline $\mathrm{C} 15-\mathrm{C} 16-\mathrm{C} 17$ & $121.4(2)$ & $\mathrm{C} 37-\mathrm{C} 38-\mathrm{C} 41$ & $120.9(2)$ \\
\hline $\mathrm{C} 17-\mathrm{C} 16-\mathrm{H} 16$ & 119.3 & $\mathrm{C} 39-\mathrm{C} 38-\mathrm{C} 37$ & $117.9(2)$ \\
\hline $\mathrm{C} 12-\mathrm{C} 17-\mathrm{H} 17$ & 119.6 & $\mathrm{C} 39-\mathrm{C} 38-\mathrm{C} 41$ & $121.3(2)$ \\
\hline $\mathrm{C} 16-\mathrm{C} 17-\mathrm{C} 12$ & $120.7(2)$ & $\mathrm{C} 38-\mathrm{C} 39-\mathrm{H} 39$ & 119.4 \\
\hline $\mathrm{C} 16-\mathrm{C} 17-\mathrm{H} 17$ & 119.6 & $\mathrm{C} 38-\mathrm{C} 39-\mathrm{C} 40$ & $121.2(2)$ \\
\hline $\mathrm{C} 15-\mathrm{C} 18-\mathrm{H} 18 \mathrm{~A}$ & 109.5 & $\mathrm{C} 40-\mathrm{C} 39-\mathrm{H} 39$ & 119.4 \\
\hline $\mathrm{C} 15-\mathrm{C} 18-\mathrm{H} 18 \mathrm{~B}$ & 109.5 & $\mathrm{C} 35-\mathrm{C} 40-\mathrm{H} 40$ & 119.5 \\
\hline $\mathrm{C} 15-\mathrm{C} 18-\mathrm{H} 18 \mathrm{C}$ & 109.5 & $\mathrm{C} 39-\mathrm{C} 40-\mathrm{C} 35$ & $121.1(2)$ \\
\hline $\mathrm{H} 18 \mathrm{~A}-\mathrm{C} 18-\mathrm{H} 18 \mathrm{~B}$ & 109.5 & $\mathrm{C} 39-\mathrm{C} 40-\mathrm{H} 40$ & 119.5 \\
\hline $\mathrm{H} 18 \mathrm{~A}-\mathrm{C} 18-\mathrm{H} 18 \mathrm{C}$ & 109.5 & $\mathrm{C} 38-\mathrm{C} 41-\mathrm{H} 41 \mathrm{~A}$ & 109.5 \\
\hline $\mathrm{H} 18 \mathrm{~B}-\mathrm{C} 18-\mathrm{H} 18 \mathrm{C}$ & 109.5 & $\mathrm{C} 38-\mathrm{C} 41-\mathrm{H} 41 \mathrm{~B}$ & 109.5 \\
\hline $\mathrm{N} 2-\mathrm{C} 19-\mathrm{C} 4$ & $114.3(2)$ & $\mathrm{C} 38-\mathrm{C} 41-\mathrm{H} 41 \mathrm{C}$ & 109.5 \\
\hline $\mathrm{N} 2-\mathrm{C} 19-\mathrm{C} 20$ & $122.3(2)$ & $\mathrm{H} 41 \mathrm{~A}-\mathrm{C} 41-\mathrm{H} 41 \mathrm{~B}$ & 109.5 \\
\hline $\mathrm{C} 20-\mathrm{C} 19-\mathrm{C} 4$ & $123.3(2)$ & $\mathrm{H} 41 \mathrm{~A}-\mathrm{C} 41-\mathrm{H} 41 \mathrm{C}$ & 109.5 \\
\hline $\mathrm{C} 19-\mathrm{C} 20-\mathrm{H} 20$ & 121.1 & $\mathrm{H} 41 \mathrm{~B}-\mathrm{C} 41-\mathrm{H} 41 \mathrm{C}$ & 109.5 \\
\hline $\mathrm{N} 1-\mathrm{C} 1-\mathrm{C} 2-\mathrm{C} 3$ & $0.1(3)$ & $\mathrm{C} 15-\mathrm{C} 16-\mathrm{C} 17-\mathrm{C} 12$ & $1.0(4)$ \\
\hline $\mathrm{N} 1-\mathrm{C} 1-\mathrm{C} 5-\mathrm{C} 6$ & $19.5(4)$ & $\mathrm{C} 17-\mathrm{C} 12-\mathrm{C} 13-\mathrm{C} 14$ & $1.5(4)$ \\
\hline $\mathrm{N} 1-\mathrm{C} 1-\mathrm{C} 5-\mathrm{C} 10$ & $-160.0(3)$ & $\mathrm{C} 18-\mathrm{C} 15-\mathrm{C} 16-\mathrm{C} 17$ & $178.2(2)$ \\
\hline $\mathrm{N} 1-\mathrm{C} 4-\mathrm{C} 19-\mathrm{N} 2$ & $-12.1(3)$ & $\mathrm{C} 19-\mathrm{N} 2-\mathrm{C} 23-\mathrm{C} 22$ & $3.1(3)$ \\
\hline $\mathrm{N} 1-\mathrm{C} 4-\mathrm{C} 19-\mathrm{C} 20$ & $165.2(2)$ & $\mathrm{C} 19-\mathrm{N} 2-\mathrm{C} 23-\mathrm{C} 24$ & $-175.5(2)$ \\
\hline $\mathrm{N} 2-\mathrm{C} 19-\mathrm{C} 20-\mathrm{C} 21$ & $-3.7(4)$ & $\mathrm{C} 19-\mathrm{C} 20-\mathrm{C} 21-\mathrm{C} 22$ & $2.2(4)$ \\
\hline $\mathrm{N} 2-\mathrm{C} 23-\mathrm{C} 24-\mathrm{N} 3$ & $-3.0(3)$ & $\mathrm{C} 20-\mathrm{C} 21-\mathrm{C} 22-\mathrm{C} 23$ & $1.7(4)$ \\
\hline $\mathrm{N} 2-\mathrm{C} 23-\mathrm{C} 24-\mathrm{C} 25$ & $176.4(3)$ & $\mathrm{C} 21-\mathrm{C} 22-\mathrm{C} 23-\mathrm{N} 2$ & $-4.5(3)$ \\
\hline $\mathrm{N} 3-\mathrm{C} 24-\mathrm{C} 25-\mathrm{C} 26$ & $0.0(3)$ & $\mathrm{C} 21-\mathrm{C} 22-\mathrm{C} 23-\mathrm{C} 24$ & $174.0(2)$ \\
\hline $\mathrm{N} 3-\mathrm{C} 24-\mathrm{C} 25-\mathrm{C} 28$ & $-178.2(2)$ & $\mathrm{C} 22-\mathrm{C} 23-\mathrm{C} 24-\mathrm{N} 3$ & $178.4(2)$ \\
\hline N3-C27-C35-C36 & $-168.1(2)$ & $\mathrm{C} 22-\mathrm{C} 23-\mathrm{C} 24-\mathrm{C} 25$ & $-2.2(4)$ \\
\hline $\mathrm{N} 3-\mathrm{C} 27-\mathrm{C} 35-\mathrm{C} 40$ & $11.4(4)$ & $\mathrm{C} 23-\mathrm{N} 2-\mathrm{C} 19-\mathrm{C} 4$ & $178.4(2)$ \\
\hline $\mathrm{C} 1-\mathrm{N} 1-\mathrm{C} 4-\mathrm{C} 3$ & $1.4(3)$ & $\mathrm{C} 23-\mathrm{N} 2-\mathrm{C} 19-\mathrm{C} 20$ & $1.0(3)$ \\
\hline $\mathrm{C} 1-\mathrm{N} 1-\mathrm{C} 4-\mathrm{C} 19$ & $-178.1(2)$ & $\mathrm{C} 23-\mathrm{C} 24-\mathrm{C} 25-\mathrm{C} 26$ & $-179.5(3)$ \\
\hline $\mathrm{C} 1-\mathrm{C} 2-\mathrm{C} 3-\mathrm{C} 4$ & $0.8(3)$ & $\mathrm{C} 23-\mathrm{C} 24-\mathrm{C} 25-\mathrm{C} 28$ & $2.3(4)$ \\
\hline $\mathrm{C} 1-\mathrm{C} 2-\mathrm{C} 3-\mathrm{C} 12$ & $-175.3(2)$ & $\mathrm{C} 24-\mathrm{N} 3-\mathrm{C} 27-\mathrm{C} 26$ & $-0.3(3)$ \\
\hline $\mathrm{C} 1-\mathrm{C} 5-\mathrm{C} 6-\mathrm{C} 7$ & $-178.8(3)$ & $\mathrm{C} 24-\mathrm{N} 3-\mathrm{C} 27-\mathrm{C} 35$ & $-179.6(2)$ \\
\hline $\mathrm{C} 1-\mathrm{C} 5-\mathrm{C} 10-\mathrm{C} 9$ & $179.1(3)$ & $\mathrm{C} 24-\mathrm{C} 25-\mathrm{C} 26-\mathrm{C} 27$ & $-0.1(3)$ \\
\hline $\mathrm{C} 2-\mathrm{C} 1-\mathrm{C} 5-\mathrm{C} 6$ & $-156.7(3)$ & $\mathrm{C} 24-\mathrm{C} 25-\mathrm{C} 28-\mathrm{C} 29$ & $-66.8(3)$ \\
\hline $\mathrm{C} 2-\mathrm{C} 1-\mathrm{C} 5-\mathrm{C} 10$ & $23.7(5)$ & $\mathrm{C} 24-\mathrm{C} 25-\mathrm{C} 28-\mathrm{C} 33$ & $111.5(3)$ \\
\hline $\mathrm{C} 2-\mathrm{C} 3-\mathrm{C} 4-\mathrm{N} 1$ & $-1.3(3)$ & $\mathrm{C} 25-\mathrm{C} 26-\mathrm{C} 27-\mathrm{N} 3$ & $0.3(3)$ \\
\hline $\mathrm{C} 2-\mathrm{C} 3-\mathrm{C} 4-\mathrm{C} 19$ & $178.1(3)$ & $\mathrm{C} 25-\mathrm{C} 26-\mathrm{C} 27-\mathrm{C} 35$ & $179.5(2)$ \\
\hline $\mathrm{C} 2-\mathrm{C} 3-\mathrm{C} 12-\mathrm{C} 13$ & $-44.1(4)$ & $\mathrm{C} 25-\mathrm{C} 28-\mathrm{C} 29-\mathrm{C} 30$ & $175.9(2)$ \\
\hline $\mathrm{C} 2-\mathrm{C} 3-\mathrm{C} 12-\mathrm{C} 17$ & $133.9(3)$ & $\mathrm{C} 25-\mathrm{C} 28-\mathrm{C} 33-\mathrm{C} 32$ & $-176.0(2)$ \\
\hline $\mathrm{C} 3-\mathrm{C} 4-\mathrm{C} 19-\mathrm{N} 2$ & $168.6(3)$ & $\mathrm{C} 26-\mathrm{C} 25-\mathrm{C} 28-\mathrm{C} 29$ & $115.4(3)$ \\
\hline $\mathrm{C} 3-\mathrm{C} 4-\mathrm{C} 19-\mathrm{C} 20$ & $-14.1(4)$ & $\mathrm{C} 26-\mathrm{C} 25-\mathrm{C} 28-\mathrm{C} 33$ & $-66.3(3)$ \\
\hline $\mathrm{C} 3-\mathrm{C} 12-\mathrm{C} 13-\mathrm{C} 14$ & $179.6(2)$ & $\mathrm{C} 26-\mathrm{C} 27-\mathrm{C} 35-\mathrm{C} 36$ & $12.8(4)$ \\
\hline $\mathrm{C} 3-\mathrm{C} 12-\mathrm{C} 17-\mathrm{C} 16$ & $-179.7(2)$ & $\mathrm{C} 26-\mathrm{C} 27-\mathrm{C} 35-\mathrm{C} 40$ & $-167.8(3)$ \\
\hline $\mathrm{C} 4-\mathrm{N} 1-\mathrm{C} 1-\mathrm{C} 2$ & $-1.0(3)$ & $\mathrm{C} 27-\mathrm{N} 3-\mathrm{C} 24-\mathrm{C} 23$ & $179.8(2)$ \\
\hline $\mathrm{C} 4-\mathrm{N} 1-\mathrm{C} 1-\mathrm{C} 5$ & $-178.1(2)$ & $\mathrm{C} 27-\mathrm{N} 3-\mathrm{C} 24-\mathrm{C} 25$ & $0.2(3)$ \\
\hline
\end{tabular}




$\begin{array}{ll}\mathrm{C} 4-\mathrm{C} 3-\mathrm{C} 12-\mathrm{C} 13 & 140.8(3) \\ \mathrm{C} 4-\mathrm{C} 3-\mathrm{C} 12-\mathrm{C} 17 & -41.2(4) \\ \mathrm{C} 4-\mathrm{C} 19-\mathrm{C} 20-\mathrm{C} 21 & 179.2(2) \\ \mathrm{C} 5-\mathrm{C} 1-\mathrm{C} 2-\mathrm{C} 3 & 176.7(3) \\ \mathrm{C} 5-\mathrm{C} 6-\mathrm{C} 7-\mathrm{C} 8 & -0.4(5) \\ \mathrm{C} 6-\mathrm{C} 5-\mathrm{C} 10-\mathrm{C} 9 & -0.4(5) \\ \mathrm{C} 6-\mathrm{C} 7-\mathrm{C} 8-\mathrm{C} 9 & -0.4(5) \\ \mathrm{C} 6-\mathrm{C} 7-\mathrm{C} 8-\mathrm{C} 11 & 179.6(3) \\ \mathrm{C} 7-\mathrm{C} 8-\mathrm{C} 9-\mathrm{C} 10 & 0.7(5) \\ \mathrm{C} 8-\mathrm{C} 9-\mathrm{C} 10-\mathrm{C} 5 & -0.3(5) \\ \mathrm{C} 10-\mathrm{C} 5-\mathrm{C} 6-\mathrm{C} 7 & 0.8(5) \\ \mathrm{C} 11-\mathrm{C} 8-\mathrm{C} 9-\mathrm{C} 10 & -179.2(3) \\ \mathrm{C} 12-\mathrm{C} 3-\mathrm{C} 4-\mathrm{N} 1 & 174.5(2) \\ \mathrm{C} 12-\mathrm{C} 3-\mathrm{C} 4-\mathrm{C} 19 & -6.1(5) \\ \mathrm{C} 12-\mathrm{C} 13-\mathrm{C} 14-\mathrm{C} 15 & -0.8(4) \\ \mathrm{C} 13-\mathrm{C} 12-\mathrm{C} 17-\mathrm{C} 16 & -1.6(4) \\ \mathrm{C} 13-\mathrm{C} 14-\mathrm{C} 15-\mathrm{C} 16 & 0.2(4) \\ \mathrm{C} 13-\mathrm{C} 14-\mathrm{C} 15-\mathrm{C} 18 & -178.3(3) \\ \mathrm{C} 14-\mathrm{C} 15-\mathrm{C} 16-\mathrm{C} 17 & -0.3(4)\end{array}$

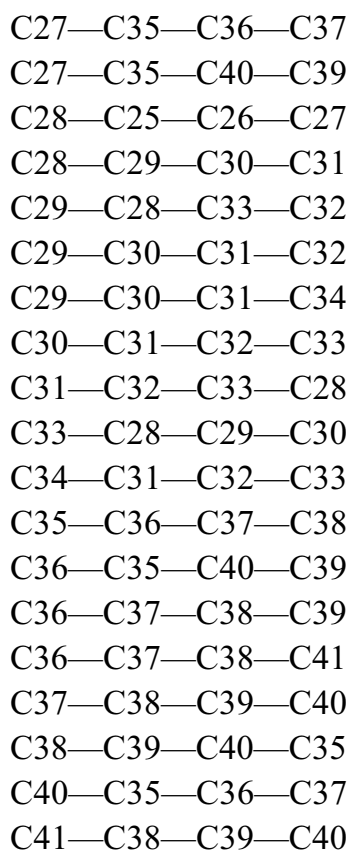

$-180.0(2)$

$-179.6(2)$

$178.0(2)$

$0.6(4)$

$2.4(4)$

$1.4(4)$

$-178.6(2)$

$-1.5(4)$

$-0.4(4)$

$-2.5(4)$

$178.5(2)$

-0.4 (4)

$-0.1(4)$

$-0.2(4)$

$178.7(2)$

$0.6(4)$

$-0.5(4)$

$0.6(4)$

$-178.3(3)$

Carbonyl $\left\{2,2^{\prime}\right.$-(pyridine-2,6-diyl)bis[3,5-bis(4-methylphenyl)pyrrolido- $\left.\left.\kappa N\right]\right\}$ nickel(II) (pyrr2PyNiCO)

Crystal data

$\left[\mathrm{Ni}\left(\mathrm{C}_{41} \mathrm{H}_{33} \mathrm{~N}_{3}\right)(\mathrm{CO})\right]$

$M_{r}=654.42$

Monoclinic, $P 12_{1} / m 1$

$a=6.6482(4) \AA$

$b=27.1709(18) \AA$

$c=9.1322(6) \AA$

$\beta=101.0700(12)^{\circ}$

$V=1618.92(18) \AA^{3}$

$Z=2$

Data collection

Bruker D8 Quest with a Photon 100 CMOS detector diffractometer

Radiation source: sealed tube Curved-graphite monochromator Detector resolution: 8 pixels $\mathrm{mm}^{-1}$ $\varphi$ and $\omega$ scans

Absorption correction: multi-scan

(SADABS; Bruker, 2016)

\section{Refinement}

Refinement on $F^{2}$

Least-squares matrix: full

$R\left[F^{2}>2 \sigma\left(F^{2}\right)\right]=0.050$

$w R\left(F^{2}\right)=0.119$

$S=1.07$

4080 reflections

222 parameters
$F(000)=684$

$D_{\mathrm{x}}=1.342 \mathrm{Mg} \mathrm{m}^{-3}$

Mo $K \alpha$ radiation, $\lambda=0.71073 \AA$

Cell parameters from 5886 reflections

$\theta=3.0-30.5^{\circ}$

$\mu=0.64 \mathrm{~mm}^{-1}$

$T=100 \mathrm{~K}$

Plates, yellow

$0.36 \times 0.27 \times 0.05 \mathrm{~mm}$

$T_{\min }=0.859, T_{\max }=1.000$

18888 measured reflections

4080 independent reflections

3280 reflections with $I>2 \sigma(I)$

$R_{\text {int }}=0.057$

$\theta_{\text {max }}=28.3^{\circ}, \theta_{\min }=3.0^{\circ}$

$h=-8 \rightarrow 8$

$k=-36 \rightarrow 36$

$l=-12 \rightarrow 12$

0 restraints

Primary atom site location: dual

Secondary atom site location: difference Fourier map

Hydrogen site location: inferred from neighbouring sites

$\mathrm{H}$-atom parameters constrained 
$w=1 /\left[\sigma^{2}\left(F_{\mathrm{o}}^{2}\right)+(0.0592 P)^{2}+0.9514 P\right]$

where $P=\left(F_{\mathrm{o}}^{2}+2 F_{\mathrm{c}}{ }^{2}\right) / 3$

$(\Delta / \sigma)_{\max }<0.001$

$$
\Delta \rho_{\max }=0.67 \text { e } \AA^{-3}
$$

\section{Special details}

Geometry. All esds (except the esd in the dihedral angle between two 1.s. planes) are estimated using the full covariance matrix. The cell esds are taken into account individually in the estimation of esds in distances, angles and torsion angles; correlations between esds in cell parameters are only used when they are defined by crystal symmetry. An approximate (isotropic) treatment of cell esds is used for estimating esds involving 1.s. planes.

Fractional atomic coordinates and isotropic or equivalent isotropic displacement parameters $\left(\AA^{2}\right)$

\begin{tabular}{|c|c|c|c|c|}
\hline & $x$ & $y$ & $z$ & $U_{\text {iso }} * / U_{\text {eq }}$ \\
\hline $\mathrm{Ni}$ & $-0.04446(6)$ & 0.750000 & $0.86705(4)$ & $0.01505(12)$ \\
\hline $\mathrm{O}$ & $-0.2636(4)$ & 0.750000 & $0.5582(3)$ & $0.0313(6)$ \\
\hline N1 & $0.1861(4)$ & 0.750000 & $1.0178(3)$ & $0.0161(5)$ \\
\hline N2 & $-0.0255(3)$ & $0.81813(7)$ & 0.89233 (19) & $0.0169(4)$ \\
\hline $\mathrm{C} 1$ & $0.5450(5)$ & 0.750000 & $1.2176(4)$ & $0.0242(7)$ \\
\hline H1 & 0.670105 & 0.750000 & 1.288274 & $0.029 *$ \\
\hline $\mathrm{C} 2$ & $0.4567(4)$ & $0.79465(9)$ & $1.1677(3)$ & $0.0218(5)$ \\
\hline $\mathrm{H} 2$ & 0.519973 & 0.824808 & 1.203580 & $0.026^{*}$ \\
\hline $\mathrm{C} 3$ & 0.2721 & $0.79438(8)$ & $1.0632(2)$ & $0.0170(4)$ \\
\hline $\mathrm{C} 4$ & $0.1516(3)$ & $0.83425(8)$ & $0.9875(2)$ & $0.0183(5)$ \\
\hline $\mathrm{C} 5$ & 0.1610 & $0.88577(8)$ & $0.9845(2)$ & $0.0185(5)$ \\
\hline C6 & -0.0168 & $0.90088(8)$ & $0.8840(2)$ & $0.0205(5)$ \\
\hline H6 & -0.055029 & 0.933942 & 0.858799 & $0.025 *$ \\
\hline $\mathrm{C} 7$ & $-0.1264(3)$ & $0.85917(8)$ & $0.8283(2)$ & $0.0179(4)$ \\
\hline $\mathrm{C} 8$ & $0.3181(3)$ & $0.91905(8)$ & $1.0691(2)$ & 0.0185 \\
\hline C9 & $0.5277(4)$ & $0.91026(9)$ & $1.0823(3)$ & $0.0221(5)$ \\
\hline H9 & 0.572595 & 0.882978 & 1.031925 & $0.027 *$ \\
\hline $\mathrm{C} 10$ & $0.6717(4)$ & $0.94084(9)$ & $1.1680(3)$ & $0.0229(5)$ \\
\hline $\mathrm{H} 10$ & 0.813288 & 0.933585 & 1.176897 & $0.028 *$ \\
\hline $\mathrm{C} 11$ & $0.6134(4)$ & $0.98169(9)$ & $1.2407(3)$ & $0.0221(5)$ \\
\hline $\mathrm{C} 12$ & $0.4036(4)$ & $0.99210(9)$ & $1.2217(3)$ & $0.0233(5)$ \\
\hline H12 & 0.359528 & 1.020579 & 1.267031 & $0.028 *$ \\
\hline C13 & $0.2591(4)$ & $0.96129(8)$ & $1.1373(3)$ & $0.0218(5)$ \\
\hline H13 & 0.117622 & 0.969085 & 1.125842 & $0.026^{*}$ \\
\hline $\mathrm{C} 14$ & $0.7677(4)$ & $1.01476(10)$ & $1.3376(3)$ & $0.0294(6)$ \\
\hline H14A & 0.906304 & 1.002061 & 1.340151 & $0.044 *$ \\
\hline H14B & 0.739580 & 1.015472 & 1.439028 & $0.044^{*}$ \\
\hline $\mathrm{H} 14 \mathrm{C}$ & 0.757029 & 1.048148 & 1.296201 & $0.044^{*}$ \\
\hline C15 & -0.3239 & $0.85618(8)$ & $0.7220(2)$ & $0.0174(4)$ \\
\hline C16 & $-0.3557(4)$ & $0.88339(8)$ & $0.5890(3)$ & $0.0210(5)$ \\
\hline H16 & -0.250400 & 0.904361 & 0.568115 & $0.025^{*}$ \\
\hline C17 & -0.5400 & $0.87994(9)$ & $0.4874(3)$ & $0.0242(5)$ \\
\hline H17 & -0.558525 & 0.898537 & 0.397741 & $0.029 *$ \\
\hline C18 & $-0.6980(4)$ & $0.84969(10)$ & $0.5148(3)$ & $0.0246(5)$ \\
\hline C19 & $-0.6676(4)$ & $0.82337(9)$ & 0.6479 & $0.0243(5)$ \\
\hline H19 & -0.773984 & 0.802848 & 0.669221 & $0.029 *$ \\
\hline
\end{tabular}




\begin{tabular}{lllll}
$\mathrm{C} 20$ & $-0.4837(4)$ & $0.82661(9)$ & $0.7504(3)$ & $0.0218(5)$ \\
$\mathrm{H} 20$ & -0.466766 & 0.808452 & 0.840810 & $0.026^{*}$ \\
$\mathrm{C} 21$ & $-0.8980(4)$ & $0.84547(13)$ & $0.4040(3)$ & $0.0388(7)$ \\
$\mathrm{H} 21 \mathrm{~A}$ & -0.898746 & 0.814872 & 0.347097 & $0.058^{*}$ \\
$\mathrm{H} 21 \mathrm{~B}$ & -0.912385 & 0.873591 & 0.335568 & $0.058^{*}$ \\
$\mathrm{H} 21 \mathrm{C}$ & -1.012360 & 0.845229 & 0.457559 & $0.058^{*}$ \\
$\mathrm{C} 22$ & $-0.2023(5)$ & 0.750000 & $0.6821(4)$ & $0.0204(7)$ \\
\hline
\end{tabular}

Atomic displacement parameters $\left(\AA^{2}\right)$

\begin{tabular}{lllllll}
\hline & $U^{11}$ & $U^{22}$ & $U^{33}$ & $U^{12}$ & $U^{13}$ & $U^{23}$ \\
\hline $\mathrm{Ni}$ & $0.0181(2)$ & $0.0143(2)$ & $0.01106(19)$ & 0.000 & $-0.00126(14)$ & 0.000 \\
$\mathrm{O}$ & $0.0447(16)$ & $0.0315(14)$ & $0.0148(12)$ & 0.000 & $-0.0018(11)$ & 0.000 \\
$\mathrm{~N} 1$ & $0.0188(13)$ & $0.0167(13)$ & $0.0115(12)$ & 0.000 & $-0.0003(10)$ & 0.000 \\
$\mathrm{~N} 2$ & $0.0214(10)$ & $0.0150(9)$ & $0.0133(9)$ & $0.0003(7)$ & $0.0003(7)$ & $-0.0019(7)$ \\
$\mathrm{C} 1$ & $0.0263(17)$ & $0.0288(18)$ & $0.0135(15)$ & 0.000 & $-0.0058(13)$ & 0.000 \\
$\mathrm{C} 2$ & $0.0247(12)$ & $0.0216(12)$ & $0.0166(11)$ & $-0.0031(9)$ & $-0.0024(9)$ & $-0.0031(9)$ \\
$\mathrm{C} 3$ & $0.0239(11)$ & $0.0160(10)$ & $0.0106(9)$ & $0.0006(8)$ & $0.0020(8)$ & $-0.0007(8)$ \\
$\mathrm{C} 4$ & $0.0195(11)$ & $0.0190(11)$ & $0.0156(10)$ & $-0.0015(9)$ & $0.0012(8)$ & $-0.0008(8)$ \\
$\mathrm{C} 5$ & $0.0216(11)$ & $0.0168(11)$ & $0.0169(10)$ & $-0.0008(9)$ & $0.0032(9)$ & $-0.0010(8)$ \\
$\mathrm{C} 6$ & $0.0242(12)$ & $0.0150(10)$ & $0.0208(11)$ & $0.0002(9)$ & $0.0004(9)$ & $0.0004(9)$ \\
$\mathrm{C} 7$ & $0.0203(11)$ & $0.0179(11)$ & $0.0145(10)$ & $0.0019(9)$ & $0.0009(8)$ & $0.0008(8)$ \\
$\mathrm{C} 8$ & $0.0235(11)$ & $0.0158(10)$ & $0.0153(10)$ & $-0.0033(9)$ & $0.0012(8)$ & $0.0011(8)$ \\
$\mathrm{C} 9$ & $0.0252(12)$ & $0.0207(11)$ & $0.0212(11)$ & $-0.0025(9)$ & $0.0066(9)$ & $-0.0023(9)$ \\
$\mathrm{C} 10$ & $0.0181(11)$ & $0.0267(12)$ & $0.0245(12)$ & $-0.0038(9)$ & $0.0053(9)$ & $0.0000(10)$ \\
$\mathrm{C} 11$ & $0.0236(12)$ & $0.0207(11)$ & $0.0209(11)$ & $-0.0062(9)$ & $0.0019(9)$ & $0.0033(9)$ \\
$\mathrm{C} 12$ & $0.0263(12)$ & $0.0151(11)$ & $0.0273(12)$ & $-0.0009(9)$ & $0.0022(10)$ & $-0.0027(9)$ \\
$\mathrm{C} 13$ & $0.0196(11)$ & $0.0164(11)$ & $0.0278(12)$ & $-0.0008(9)$ & $0.0001(9)$ & $0.0005(9)$ \\
$\mathrm{C} 14$ & $0.0248(13)$ & $0.0307(14)$ & $0.0307(13)$ & $-0.0071(10)$ & $0.0009(10)$ & $-0.0061(11)$ \\
$\mathrm{C} 15$ & $0.0198(11)$ & $0.0157(10)$ & $0.0157(10)$ & $0.0025(8)$ & $0.0007(8)$ & $-0.0027(8)$ \\
$\mathrm{C} 16$ & $0.0256(12)$ & $0.0166(11)$ & $0.0207(11)$ & $0.0024(9)$ & $0.0039(9)$ & $0.0004(9)$ \\
$\mathrm{C} 17$ & $0.0288(13)$ & $0.0256(12)$ & $0.0171(11)$ & $0.0091(10)$ & $0.0022(9)$ & $0.0021(9)$ \\
$\mathrm{C} 18$ & $0.0214(12)$ & $0.0330(14)$ & $0.0185(11)$ & $0.0066(10)$ & $0.0017(9)$ & $-0.0026(10)$ \\
$\mathrm{C} 19$ & $0.0213(12)$ & $0.0306(13)$ & $0.0215(12)$ & $-0.0017(10)$ & $0.0051(9)$ & $-0.0017(10)$ \\
$\mathrm{C} 20$ & $0.0233(12)$ & $0.0236(12)$ & $0.0183(11)$ & $0.0022(9)$ & $0.0037(9)$ & $0.0012(9)$ \\
$\mathrm{C} 21$ & $0.0231(13)$ & $0.067(2)$ & $0.0243(13)$ & $0.0050(13)$ & $0.0001(10)$ & $0.0025(14)$ \\
$\mathrm{C} 22$ & $0.0260(17)$ & $0.0165(15)$ & $0.0185(16)$ & 0.000 & $0.0039(13)$ & 0.000 \\
& & & & & & \\
\hline & & & & & &
\end{tabular}

Geometric parameters ( $\left.\AA,{ }^{\circ}\right)$

\begin{tabular}{llll}
\hline $\mathrm{Ni}-\mathrm{N} 1$ & $1.853(3)$ & $\mathrm{C} 10-\mathrm{H} 10$ & 0.9500 \\
$\mathrm{Ni}-\mathrm{N} 2^{\mathrm{i}}$ & $1.8667(18)$ & $\mathrm{C} 10-\mathrm{C} 11$ & $1.386(3)$ \\
$\mathrm{Ni}-\mathrm{N} 2$ & $1.8666(18)$ & $\mathrm{C} 11-\mathrm{C} 12$ & $1.401(3)$ \\
$\mathrm{Ni}-\mathrm{C} 22$ & $1.809(3)$ & $\mathrm{C} 11-\mathrm{C} 14$ & $1.515(3)$ \\
$\mathrm{O}-\mathrm{C} 22$ & $1.126(4)$ & $\mathrm{C} 12-\mathrm{H} 12$ & 0.9500 \\
$\mathrm{~N} 1-\mathrm{C} 3^{\mathrm{i}}$ & $1.364(2)$ & $\mathrm{C} 12-\mathrm{C} 13$ & $1.391(3)$ \\
$\mathrm{N} 1-\mathrm{C} 3$ & $1.364(2)$ & $\mathrm{C} 13-\mathrm{H} 13$ & 0.9500 \\
$\mathrm{~N} 2-\mathrm{C} 4$ & $1.393(3)$ & $\mathrm{C} 14-\mathrm{H} 14 \mathrm{~A}$ & 0.9800
\end{tabular}




\begin{tabular}{|c|c|c|c|}
\hline $\mathrm{N} 2-\mathrm{C} 7$ & $1.373(3)$ & C14-H14B & 0.9800 \\
\hline $\mathrm{C} 1-\mathrm{H} 1$ & 0.9500 & $\mathrm{C} 14-\mathrm{H} 14 \mathrm{C}$ & 0.9800 \\
\hline $\mathrm{C} 1-\mathrm{C} 2$ & $1.386(3)$ & $\mathrm{C} 15-\mathrm{C} 16$ & $1.402(3)$ \\
\hline $\mathrm{C} 1-\mathrm{C} 2^{\mathrm{i}}$ & $1.386(3)$ & $\mathrm{C} 15-\mathrm{C} 20$ & $1.395(3)$ \\
\hline $\mathrm{C} 2-\mathrm{H} 2$ & 0.9500 & $\mathrm{C} 16-\mathrm{H} 16$ & 0.9500 \\
\hline $\mathrm{C} 2-\mathrm{C} 3$ & $1.403(3)$ & $\mathrm{C} 16-\mathrm{C} 17$ & $1.391(3)$ \\
\hline $\mathrm{C} 3-\mathrm{C} 4$ & $1.442(3)$ & $\mathrm{C} 17-\mathrm{H} 17$ & 0.9500 \\
\hline $\mathrm{C} 4-\mathrm{C} 5$ & $1.402(3)$ & $\mathrm{C} 17-\mathrm{C} 18$ & $1.394(4)$ \\
\hline $\mathrm{C} 5-\mathrm{C} 6$ & $1.411(3)$ & $\mathrm{C} 18-\mathrm{C} 19$ & $1.391(3)$ \\
\hline $\mathrm{C} 5-\mathrm{C} 8$ & $1.482(3)$ & $\mathrm{C} 18-\mathrm{C} 21$ & $1.512(3)$ \\
\hline $\mathrm{C} 6-\mathrm{H} 6$ & 0.9500 & $\mathrm{C} 19-\mathrm{H} 19$ & 0.9500 \\
\hline $\mathrm{C} 6-\mathrm{C} 7$ & $1.390(3)$ & $\mathrm{C} 19-\mathrm{C} 20$ & $1.392(3)$ \\
\hline $\mathrm{C} 7-\mathrm{C} 15$ & $1.478(3)$ & $\mathrm{C} 20-\mathrm{H} 20$ & 0.9500 \\
\hline $\mathrm{C} 8-\mathrm{C} 9$ & $1.396(3)$ & $\mathrm{C} 21-\mathrm{H} 21 \mathrm{~A}$ & 0.9800 \\
\hline $\mathrm{C} 8-\mathrm{C} 13$ & $1.398(3)$ & $\mathrm{C} 21-\mathrm{H} 21 \mathrm{~B}$ & 0.9800 \\
\hline C9-H9 & 0.9500 & $\mathrm{C} 21-\mathrm{H} 21 \mathrm{C}$ & 0.9800 \\
\hline $\mathrm{C} 9-\mathrm{C} 10$ & $1.390(3)$ & & \\
\hline $\mathrm{N} 1-\mathrm{Ni}-\mathrm{N} 2$ & $83.20(6)$ & $\mathrm{C} 11-\mathrm{C} 10-\mathrm{H} 10$ & 119.2 \\
\hline $\mathrm{N} 1-\mathrm{Ni}-\mathrm{N} 2^{\mathrm{i}}$ & $83.20(6)$ & $\mathrm{C} 10-\mathrm{C} 11-\mathrm{C} 12$ & $117.7(2)$ \\
\hline $\mathrm{N} 2-\mathrm{Ni}-\mathrm{N} 2^{\mathrm{i}}$ & $165.18(11)$ & $\mathrm{C} 10-\mathrm{C} 11-\mathrm{C} 14$ & $122.3(2)$ \\
\hline $\mathrm{C} 22-\mathrm{Ni}-\mathrm{N} 1$ & $160.41(13)$ & $\mathrm{C} 12-\mathrm{C} 11-\mathrm{C} 14$ & $119.9(2)$ \\
\hline $\mathrm{C} 22-\mathrm{Ni}-\mathrm{N} 2$ & $97.39(5)$ & $\mathrm{C} 11-\mathrm{C} 12-\mathrm{H} 12$ & 119.6 \\
\hline $\mathrm{C} 22-\mathrm{Ni}-\mathrm{N} 2^{\mathrm{i}}$ & $97.40(6)$ & $\mathrm{C} 13-\mathrm{C} 12-\mathrm{C} 11$ & $120.8(2)$ \\
\hline $\mathrm{C} 3{ }^{\mathrm{i}}-\mathrm{N} 1-\mathrm{Ni}$ & $117.66(13)$ & $\mathrm{C} 13-\mathrm{C} 12-\mathrm{H} 12$ & 119.6 \\
\hline $\mathrm{C} 3-\mathrm{N} 1-\mathrm{Ni}$ & $117.66(13)$ & $\mathrm{C} 8-\mathrm{C} 13-\mathrm{H} 13$ & 119.4 \\
\hline 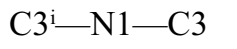 & $124.2(3)$ & $\mathrm{C} 12-\mathrm{C} 13-\mathrm{C} 8$ & $121.2(2)$ \\
\hline $\mathrm{C} 4-\mathrm{N} 2-\mathrm{Ni}$ & $114.70(15)$ & $\mathrm{C} 12-\mathrm{C} 13-\mathrm{H} 13$ & 119.4 \\
\hline $\mathrm{C} 7-\mathrm{N} 2-\mathrm{Ni}$ & $137.48(15)$ & $\mathrm{C} 11-\mathrm{C} 14-\mathrm{H} 14 \mathrm{~A}$ & 109.5 \\
\hline $\mathrm{C} 7-\mathrm{N} 2-\mathrm{C} 4$ & $107.19(18)$ & $\mathrm{C} 11-\mathrm{C} 14-\mathrm{H} 14 \mathrm{~B}$ & 109.5 \\
\hline $\mathrm{C} 2-\mathrm{C} 1-\mathrm{H} 1$ & 118.9 & $\mathrm{C} 11-\mathrm{C} 14-\mathrm{H} 14 \mathrm{C}$ & 109.5 \\
\hline $\mathrm{C} 22^{\mathrm{i}}-\mathrm{C} 1-\mathrm{H} 1$ & 118.9 & $\mathrm{H} 14 \mathrm{~A}-\mathrm{C} 14-\mathrm{H} 14 \mathrm{~B}$ & 109.5 \\
\hline $\mathrm{C} 22^{\mathrm{i}}-\mathrm{C} 1-\mathrm{C} 2$ & $122.2(3)$ & $\mathrm{H} 14 \mathrm{~A}-\mathrm{C} 14-\mathrm{H} 14 \mathrm{C}$ & 109.5 \\
\hline $\mathrm{C} 1-\mathrm{C} 2-\mathrm{H} 2$ & 120.7 & $\mathrm{H} 14 \mathrm{~B}-\mathrm{C} 14-\mathrm{H} 14 \mathrm{C}$ & 109.5 \\
\hline $\mathrm{C} 1-\mathrm{C} 2-\mathrm{C} 3$ & $118.6(2)$ & $\mathrm{C} 16-\mathrm{C} 15-\mathrm{C} 7$ & $120.5(2)$ \\
\hline $\mathrm{C} 3-\mathrm{C} 2-\mathrm{H} 2$ & 120.7 & $\mathrm{C} 20-\mathrm{C} 15-\mathrm{C} 7$ & $121.4(2)$ \\
\hline $\mathrm{N} 1-\mathrm{C} 3-\mathrm{C} 2$ & $118.2(2)$ & $\mathrm{C} 20-\mathrm{C} 15-\mathrm{C} 16$ & $118.1(2)$ \\
\hline $\mathrm{N} 1-\mathrm{C} 3-\mathrm{C} 4$ & $110.88(19)$ & $\mathrm{C} 15-\mathrm{C} 16-\mathrm{H} 16$ & 119.7 \\
\hline $\mathrm{C} 2-\mathrm{C} 3-\mathrm{C} 4$ & $130.9(2)$ & $\mathrm{C} 17-\mathrm{C} 16-\mathrm{C} 15$ & $120.6(2)$ \\
\hline $\mathrm{N} 2-\mathrm{C} 4-\mathrm{C} 3$ & $112.82(19)$ & $\mathrm{C} 17-\mathrm{C} 16-\mathrm{H} 16$ & 119.7 \\
\hline $\mathrm{N} 2-\mathrm{C} 4-\mathrm{C} 5$ & $109.72(19)$ & $\mathrm{C} 16-\mathrm{C} 17-\mathrm{H} 17$ & 119.4 \\
\hline $\mathrm{C} 5-\mathrm{C} 4-\mathrm{C} 3$ & $137.4(2)$ & $\mathrm{C} 16-\mathrm{C} 17-\mathrm{C} 18$ & $121.2(2)$ \\
\hline $\mathrm{C} 4-\mathrm{C} 5-\mathrm{C} 6$ & $105.6(2)$ & $\mathrm{C} 18-\mathrm{C} 17-\mathrm{H} 17$ & 119.4 \\
\hline $\mathrm{C} 4-\mathrm{C} 5-\mathrm{C} 8$ & $129.0(2)$ & $\mathrm{C} 17-\mathrm{C} 18-\mathrm{C} 21$ & $121.4(2)$ \\
\hline $\mathrm{C} 6-\mathrm{C} 5-\mathrm{C} 8$ & $125.5(2)$ & $\mathrm{C} 19-\mathrm{C} 18-\mathrm{C} 17$ & $118.1(2)$ \\
\hline $\mathrm{C} 5-\mathrm{C} 6-\mathrm{H} 6$ & 125.8 & $\mathrm{C} 19-\mathrm{C} 18-\mathrm{C} 21$ & $120.5(2)$ \\
\hline $\mathrm{C} 7-\mathrm{C} 6-\mathrm{C} 5$ & $108.4(2)$ & $\mathrm{C} 18-\mathrm{C} 19-\mathrm{H} 19$ & 119.4 \\
\hline $\mathrm{C} 7-\mathrm{C} 6-\mathrm{H} 6$ & 125.8 & $\mathrm{C} 18-\mathrm{C} 19-\mathrm{C} 20$ & $121.1(2)$ \\
\hline
\end{tabular}




\begin{tabular}{|c|c|c|c|}
\hline $\mathrm{N} 2-\mathrm{C} 7-\mathrm{C} 6$ & $109.10(19)$ & $\mathrm{C} 20-\mathrm{C} 19-\mathrm{H} 19$ & 119.4 \\
\hline $\mathrm{N} 2-\mathrm{C} 7-\mathrm{C} 15$ & $122.3(2)$ & $\mathrm{C} 15-\mathrm{C} 20-\mathrm{H} 20$ & 119.6 \\
\hline $\mathrm{C} 6-\mathrm{C} 7-\mathrm{C} 15$ & $128.5(2)$ & $\mathrm{C} 19-\mathrm{C} 20-\mathrm{C} 15$ & $120.9(2)$ \\
\hline $\mathrm{C} 9-\mathrm{C} 8-\mathrm{C} 5$ & $122.2(2)$ & $\mathrm{C} 19-\mathrm{C} 20-\mathrm{H} 20$ & 119.6 \\
\hline $\mathrm{C} 9-\mathrm{C} 8-\mathrm{C} 13$ & $117.6(2)$ & $\mathrm{C} 18-\mathrm{C} 21-\mathrm{H} 21 \mathrm{~A}$ & 109.5 \\
\hline $\mathrm{C} 13-\mathrm{C} 8-\mathrm{C} 5$ & $120.2(2)$ & $\mathrm{C} 18-\mathrm{C} 21-\mathrm{H} 21 \mathrm{~B}$ & 109.5 \\
\hline $\mathrm{C} 8-\mathrm{C} 9-\mathrm{H} 9$ & 119.5 & $\mathrm{C} 18-\mathrm{C} 21-\mathrm{H} 21 \mathrm{C}$ & 109.5 \\
\hline $\mathrm{C} 10-\mathrm{C} 9-\mathrm{C} 8$ & $121.0(2)$ & $\mathrm{H} 21 \mathrm{~A}-\mathrm{C} 21-\mathrm{H} 21 \mathrm{~B}$ & 109.5 \\
\hline $\mathrm{C} 10-\mathrm{C} 9-\mathrm{H} 9$ & 119.5 & $\mathrm{H} 21 \mathrm{~A}-\mathrm{C} 21-\mathrm{H} 21 \mathrm{C}$ & 109.5 \\
\hline $\mathrm{C} 9-\mathrm{C} 10-\mathrm{H} 10$ & 119.2 & $\mathrm{H} 21 \mathrm{~B}-\mathrm{C} 21-\mathrm{H} 21 \mathrm{C}$ & 109.5 \\
\hline $\mathrm{C} 11-\mathrm{C} 10-\mathrm{C} 9$ & $121.5(2)$ & $\mathrm{O}-\mathrm{C} 22-\mathrm{Ni}$ & $166.1(3)$ \\
\hline $\mathrm{Ni}-\mathrm{N} 1-\mathrm{C} 3-\mathrm{C} 2$ & $-174.47(18)$ & $\mathrm{C} 4-\mathrm{C} 5-\mathrm{C} 8-\mathrm{C} 9$ & $45.6(4)$ \\
\hline $\mathrm{Ni}-\mathrm{N} 1-\mathrm{C} 3-\mathrm{C} 4$ & $4.0(3)$ & $\mathrm{C} 4-\mathrm{C} 5-\mathrm{C} 8-\mathrm{C} 13$ & $-135.3(3)$ \\
\hline $\mathrm{Ni}-\mathrm{N} 2-\mathrm{C} 4-\mathrm{C} 3$ & $-7.8(2)$ & $\mathrm{C} 5-\mathrm{C} 6-\mathrm{C} 7-\mathrm{N} 2$ & $1.1(3)$ \\
\hline $\mathrm{Ni}-\mathrm{N} 2-\mathrm{C} 4-\mathrm{C} 5$ & $173.14(15)$ & $\mathrm{C} 5-\mathrm{C} 6-\mathrm{C} 7-\mathrm{C} 15$ & $178.8(2)$ \\
\hline $\mathrm{Ni}-\mathrm{N} 2-\mathrm{C} 7-\mathrm{C} 6$ & $-170.94(18)$ & $\mathrm{C} 5-\mathrm{C} 8-\mathrm{C} 9-\mathrm{C} 10$ & $-177.0(2)$ \\
\hline $\mathrm{Ni}-\mathrm{N} 2-\mathrm{C} 7-\mathrm{C} 15$ & $11.1(4)$ & $\mathrm{C} 5-\mathrm{C} 8-\mathrm{C} 13-\mathrm{C} 12$ & $177.7(2)$ \\
\hline $\mathrm{N} 1-\mathrm{Ni}-\mathrm{N} 2-\mathrm{C} 4$ & $7.95(17)$ & $\mathrm{C} 6-\mathrm{C} 5-\mathrm{C} 8-\mathrm{C} 9$ & $-135.8(2)$ \\
\hline $\mathrm{N} 1-\mathrm{Ni}-\mathrm{N} 2-\mathrm{C} 7$ & $177.3(2)$ & $\mathrm{C} 6-\mathrm{C} 5-\mathrm{C} 8-\mathrm{C} 13$ & $43.2(3)$ \\
\hline $\mathrm{N} 1-\mathrm{Ni}-\mathrm{C} 22-\mathrm{O}$ & $0.000(5)$ & $\mathrm{C} 6-\mathrm{C} 7-\mathrm{C} 15-\mathrm{C} 16$ & $48.5(3)$ \\
\hline $\mathrm{N} 1-\mathrm{C} 3-\mathrm{C} 4-\mathrm{N} 2$ & $2.5(3)$ & $\mathrm{C} 6-\mathrm{C} 7-\mathrm{C} 15-\mathrm{C} 20$ & $-131.3(3)$ \\
\hline $\mathrm{N} 1-\mathrm{C} 3-\mathrm{C} 4-\mathrm{C} 5$ & $-178.9(3)$ & $\mathrm{C} 7-\mathrm{N} 2-\mathrm{C} 4-\mathrm{C} 3$ & $179.68(18)$ \\
\hline $\mathrm{N} 2^{\mathrm{i}}-\mathrm{Ni}-\mathrm{N} 1-\mathrm{C} 3^{\mathrm{i}}$ & $6.7(2)$ & $\mathrm{C} 7-\mathrm{N} 2-\mathrm{C} 4-\mathrm{C} 5$ & $0.7(3)$ \\
\hline $\mathrm{N} 2 \mathrm{i}-\mathrm{Ni}-\mathrm{N} 1-\mathrm{C} 3$ & $179.2(2)$ & $\mathrm{C} 7-\mathrm{C} 15-\mathrm{C} 16-\mathrm{C} 17$ & $178.8(2)$ \\
\hline $\mathrm{N} 2-\mathrm{Ni}-\mathrm{N} 1-\mathrm{C} 3$ & $-6.7(2)$ & $\mathrm{C} 7-\mathrm{C} 15-\mathrm{C} 20-\mathrm{C} 19$ & $-178.7(2)$ \\
\hline $\mathrm{N} 2-\mathrm{Ni}-\mathrm{N} 1-\mathrm{C} 3^{\mathrm{i}}$ & $-179.2(2)$ & $\mathrm{C} 8-\mathrm{C} 5-\mathrm{C} 6-\mathrm{C} 7$ & $-179.5(2)$ \\
\hline $\mathrm{N} 2 \mathrm{i}-\mathrm{Ni}-\mathrm{N} 2-\mathrm{C} 4$ & $31.5(6)$ & $\mathrm{C} 8-\mathrm{C} 9-\mathrm{C} 10-\mathrm{C} 11$ & $-1.4(4)$ \\
\hline $\mathrm{N} 2 \mathrm{i}-\mathrm{Ni}-\mathrm{N} 2-\mathrm{C} 7$ & $-159.1(3)$ & $\mathrm{C} 9-\mathrm{C} 8-\mathrm{C} 13-\mathrm{C} 12$ & $-3.2(3)$ \\
\hline $\mathrm{N} 2-\mathrm{Ni}-\mathrm{C} 22-\mathrm{O}$ & $90.49(6)$ & $\mathrm{C} 9-\mathrm{C} 10-\mathrm{C} 11-\mathrm{C} 12$ & $-1.8(4)$ \\
\hline $\mathrm{N} 2 \mathrm{i}-\mathrm{Ni}-\mathrm{C} 22-\mathrm{O}$ & $-90.49(6)$ & $\mathrm{C} 9-\mathrm{C} 10-\mathrm{C} 11-\mathrm{C} 14$ & $178.5(2)$ \\
\hline $\mathrm{N} 2-\mathrm{C} 4-\mathrm{C} 5-\mathrm{C} 6$ & $0.0(3)$ & $\mathrm{C} 10-\mathrm{C} 11-\mathrm{C} 12-\mathrm{C} 13$ & $2.4(4)$ \\
\hline $\mathrm{N} 2-\mathrm{C} 4-\mathrm{C} 5-\mathrm{C} 8$ & $178.8(2)$ & $\mathrm{C} 11-\mathrm{C} 12-\mathrm{C} 13-\mathrm{C} 8$ & $0.1(4)$ \\
\hline $\mathrm{N} 2-\mathrm{C} 7-\mathrm{C} 15-\mathrm{C} 16$ & $-134.0(2)$ & $\mathrm{C} 13-\mathrm{C} 8-\mathrm{C} 9-\mathrm{C} 10$ & $3.9(3)$ \\
\hline $\mathrm{N} 2-\mathrm{C} 7-\mathrm{C} 15-\mathrm{C} 20$ & $46.2(3)$ & $\mathrm{C} 14-\mathrm{C} 11-\mathrm{C} 12-\mathrm{C} 13$ & $-177.8(2)$ \\
\hline $\mathrm{C} 1-\mathrm{C} 2-\mathrm{C} 3-\mathrm{N} 1$ & $1.2(4)$ & $\mathrm{C} 15-\mathrm{C} 16-\mathrm{C} 17-\mathrm{C} 18$ & $0.3(4)$ \\
\hline $\mathrm{C} 1-\mathrm{C} 2-\mathrm{C} 3-\mathrm{C} 4$ & $-176.9(3)$ & $\mathrm{C} 16-\mathrm{C} 15-\mathrm{C} 20-\mathrm{C} 19$ & $1.5(3)$ \\
\hline $\mathrm{C} 22^{\mathrm{i}}-\mathrm{C} 1-\mathrm{C} 2-\mathrm{C} 3$ & $-0.1(5)$ & $\mathrm{C} 16-\mathrm{C} 17-\mathrm{C} 18-\mathrm{C} 19$ & $0.8(4)$ \\
\hline $\mathrm{C} 2-\mathrm{C} 3-\mathrm{C} 4-\mathrm{N} 2$ & $-179.3(2)$ & $\mathrm{C} 16-\mathrm{C} 17-\mathrm{C} 18-\mathrm{C} 21$ & $-179.4(2)$ \\
\hline $\mathrm{C} 2-\mathrm{C} 3-\mathrm{C} 4-\mathrm{C} 5$ & $-0.6(5)$ & $\mathrm{C} 17-\mathrm{C} 18-\mathrm{C} 19-\mathrm{C} 20$ & $-0.8(4)$ \\
\hline $\mathrm{C} 33^{\mathrm{i}}-\mathrm{N} 1-\mathrm{C} 3-\mathrm{C} 2$ & $-2.6(4)$ & $\mathrm{C} 18-\mathrm{C} 19-\mathrm{C} 20-\mathrm{C} 15$ & $-0.4(4)$ \\
\hline $\mathrm{C} 33^{\mathrm{i}}-\mathrm{N} 1-\mathrm{C} 3-\mathrm{C} 4$ & $175.9(2)$ & $\mathrm{C} 20-\mathrm{C} 15-\mathrm{C} 16-\mathrm{C} 17$ & $-1.4(3)$ \\
\hline $\mathrm{C} 3-\mathrm{C} 4-\mathrm{C} 5-\mathrm{C} 6$ & $-178.7(3)$ & $\mathrm{C} 21-\mathrm{C} 18-\mathrm{C} 19-\mathrm{C} 20$ & $179.5(2)$ \\
\hline $\mathrm{C} 3-\mathrm{C} 4-\mathrm{C} 5-\mathrm{C} 8$ & $0.1(5)$ & $\mathrm{C} 22-\mathrm{Ni}-\mathrm{N} 1-\mathrm{C} 3^{\mathrm{i}}$ & $-86.2(2)$ \\
\hline $\mathrm{C} 4-\mathrm{N} 2-\mathrm{C} 7-\mathrm{C} 6$ & $-1.1(3)$ & $\mathrm{C} 22-\mathrm{Ni}-\mathrm{N} 1-\mathrm{C} 3$ & $86.2(2)$ \\
\hline
\end{tabular}




\begin{tabular}{llll}
$\mathrm{C} 4-\mathrm{N} 2-\mathrm{C} 7-\mathrm{C} 15$ & $-179.0(2)$ & $\mathrm{C} 22-\mathrm{Ni}-\mathrm{N} 2-\mathrm{C} 4$ & $-152.32(18)$ \\
$\mathrm{C} 4-\mathrm{C} 5-\mathrm{C} 6-\mathrm{C} 7$ & $-0.7(3)$ & $\mathrm{C} 22-\mathrm{Ni}-\mathrm{N} 2-\mathrm{C} 7$ & $17.0(3)$ \\
\hline
\end{tabular}

Symmetry code: (i) $x,-y+3 / 2, z$.

Ammine $\left\{2,2^{\prime}\right.$-(pyridine-2,6-diyl)bis[3,5-bis(4-methylphenyl)pyrrolido- $k$ N]\}nickel(II) (pyrr2PyNiNH3)

\section{Crystal data}

$\left[\mathrm{Ni}\left(\mathrm{C}_{41} \mathrm{H}_{33} \mathrm{~N}_{3}\right)\left(\mathrm{NH}_{3}\right)\right]$

$M_{r}=643.45$

Monoclinic, $P 2_{1} / c$

$a=15.9773$ (6) $\AA$

$b=14.9441(5) \AA$

$c=14.3238(5) \AA$

$\beta=107.8140(8)^{\circ}$

$V=3256.1(2) \AA^{3}$

$Z=4$

\section{Data collection}

Bruker D8 Quest with a Photon 100 CMOS detector diffractometer

Curved-graphite monochromator $\varphi$ and $\omega$ scans

Absorption correction: multi-scan

(SADABS; Bruker, 2016)

$T_{\text {min }}=0.858, T_{\text {max }}=0.967$

\section{Refinement}

Refinement on $F^{2}$

Least-squares matrix: full

$R\left[F^{2}>2 \sigma\left(F^{2}\right)\right]=0.036$

$w R\left(F^{2}\right)=0.098$

$S=1.05$

9931 reflections

431 parameters

0 restraints

Primary atom site location: dual

\author{
$F(000)=1352$ \\ $D_{\mathrm{x}}=1.313 \mathrm{Mg} \mathrm{m}^{-3}$ \\ Mo $K \alpha$ radiation, $\lambda=0.71073 \AA$ \\ Cell parameters from 9841 reflections \\ $\theta=3.0-39.4^{\circ}$ \\ $\mu=0.63 \mathrm{~mm}^{-1}$ \\ $T=100 \mathrm{~K}$ \\ Plates, red \\ $0.46 \times 0.41 \times 0.14 \mathrm{~mm}$
}

\section{Special details}

Geometry. All esds (except the esd in the dihedral angle between two 1.s. planes) are estimated using the full covariance matrix. The cell esds are taken into account individually in the estimation of esds in distances, angles and torsion angles; correlations between esds in cell parameters are only used when they are defined by crystal symmetry. An approximate (isotropic) treatment of cell esds is used for estimating esds involving l.s. planes.

Fractional atomic coordinates and isotropic or equivalent isotropic displacement parameters $\left(\hat{A}^{2}\right)$

\begin{tabular}{lllll}
\hline & $x$ & $y$ & $z$ & $U_{\text {iso }} * / U_{\text {eq }}$ \\
\hline $\mathrm{Ni}$ & $0.10799(2)$ & $0.41253(2)$ & $0.93990(2)$ & $0.01428(5)$ \\
$\mathrm{N} 1$ & $0.21118(7)$ & $0.43570(7)$ & $0.90627(7)$ & $0.01684(19)$ \\
$\mathrm{N} 2$ & $0.05856(7)$ & $0.39854(7)$ & $0.80601(7)$ & $0.01515(19)$ \\
$\mathrm{N} 3$ & $-0.00188(7)$ & $0.36827(7)$ & $0.94266(7)$ & $0.01631(19)$ \\
$\mathrm{N} 4$ & $0.14688(8)$ & $0.46458(8)$ & $1.06929(8)$ & $0.0179(2)$
\end{tabular}




\begin{tabular}{|c|c|c|c|c|}
\hline $\mathrm{H} 4 \mathrm{~A}$ & $0.1037(17)$ & 0.4707 (17) & $1.0960(18)$ & $0.060(7)^{*}$ \\
\hline H4B & $0.1871(18)$ & 0.4399 (19) & $1.1142(19)$ & $0.063(7)^{*}$ \\
\hline $\mathrm{H} 4 \mathrm{C}$ & $0.1692(16)$ & $0.5156(18)$ & $1.0630(17)$ & $0.056(7)^{*}$ \\
\hline $\mathrm{C} 1$ & $0.29896(8)$ & $0.45061(9)$ & $0.94997(9)$ & $0.0190(2)$ \\
\hline $\mathrm{C} 2$ & $0.33888(8)$ & $0.47073(10)$ & $0.87809(10)$ & $0.0227(3)$ \\
\hline $\mathrm{H} 2$ & 0.399240 & 0.484362 & 0.889319 & $0.027 *$ \\
\hline $\mathrm{C} 3$ & $0.27366(8)$ & $0.46719(9)$ & $0.78618(9)$ & $0.0188(2)$ \\
\hline $\mathrm{C} 4$ & $0.19592(8)$ & $0.44495(9)$ & 0.80609 (9) & $0.0171(2)$ \\
\hline $\mathrm{C} 5$ & $0.10722(8)$ & $0.42434(8)$ & 0.74805 (9) & $0.0162(2)$ \\
\hline C6 & $0.06831(9)$ & $0.42720(9)$ & $0.64636(9)$ & $0.0185(2)$ \\
\hline H6 & 0.101605 & 0.443252 & 0.604162 & $0.022 *$ \\
\hline $\mathrm{C} 7$ & $-0.02032(9)$ & $0.40596(9)$ & $0.60869(9)$ & $0.0200(2)$ \\
\hline H7 & -0.047903 & 0.408038 & 0.539816 & $0.024 *$ \\
\hline $\mathrm{C} 8$ & $-0.06959(8)$ & $0.38168(9)$ & $0.66984(9)$ & $0.0190(2)$ \\
\hline H8 & -0.130417 & 0.368353 & 0.643358 & $0.023 *$ \\
\hline C9 & $-0.02792(8)$ & $0.37728(8)$ & 0.77075 (9) & $0.0159(2)$ \\
\hline $\mathrm{C} 10$ & $-0.06230(8)$ & $0.35450(8)$ & $0.85048(9)$ & $0.0162(2)$ \\
\hline C11 & $-0.14162(8)$ & $0.32195(8)$ & $0.86028(9)$ & $0.0170(2)$ \\
\hline $\mathrm{C} 12$ & $-0.12798(8)$ & $0.31616(9)$ & 0.96247 (9) & $0.0186(2)$ \\
\hline H12 & -0.169888 & 0.296952 & 0.993071 & $0.022 *$ \\
\hline $\mathrm{C} 13$ & $-0.04184(8)$ & $0.34373(8)$ & 1.01047 (9) & $0.0172(2)$ \\
\hline $\mathrm{C} 14$ & $0.34369(8)$ & $0.43631(9)$ & $1.05496(9)$ & $0.0196(2)$ \\
\hline C15 & $0.32402(9)$ & $0.36205(10)$ & $1.10428(10)$ & $0.0225(3)$ \\
\hline H15 & 0.278667 & 0.322055 & 1.070416 & $0.027 *$ \\
\hline $\mathrm{C} 16$ & $0.37015(10)$ & $0.34625(10)$ & $1.20222(10)$ & $0.0264(3)$ \\
\hline H16 & 0.354631 & 0.296548 & 1.234851 & $0.032 *$ \\
\hline $\mathrm{C} 17$ & $0.43869(9)$ & 0.40205 (11) & $1.25325(10)$ & $0.0273(3)$ \\
\hline $\mathrm{C} 18$ & $0.45818(9)$ & $0.47588(11)$ & 1.20459 (11) & $0.0274(3)$ \\
\hline H18 & 0.504218 & 0.515165 & 1.238430 & $0.033 *$ \\
\hline C19 & $0.41132(9)$ & $0.49320(10)$ & $1.10702(10)$ & $0.0233(3)$ \\
\hline H19 & 0.425507 & 0.544340 & 1.075466 & $0.028^{*}$ \\
\hline $\mathrm{C} 20$ & $0.49213(12)$ & $0.38050(14)$ & $1.35736(12)$ & 0.0393 (4) \\
\hline $\mathrm{H} 20 \mathrm{~A}$ & 0.458982 & 0.339379 & 1.386063 & $0.059 *$ \\
\hline $\mathrm{H} 20 \mathrm{~B}$ & 0.504500 & 0.435775 & 1.395978 & $0.059^{*}$ \\
\hline $\mathrm{H} 20 \mathrm{C}$ & 0.547612 & 0.352361 & 1.357653 & $0.059^{*}$ \\
\hline $\mathrm{C} 21$ & $0.28514(8)$ & $0.48099(9)$ & $0.68838(9)$ & $0.0186(2)$ \\
\hline $\mathrm{C} 22$ & $0.32480(11)$ & $0.41602(10)$ & 0.64649 (11) & $0.0266(3)$ \\
\hline $\mathrm{H} 22$ & 0.346065 & 0.362588 & 0.681707 & $0.032 *$ \\
\hline $\mathrm{C} 23$ & $0.33372(12)$ & $0.42831(11)$ & 0.55354 (11) & $0.0308(3)$ \\
\hline $\mathrm{H} 23$ & 0.360985 & 0.383006 & 0.526433 & $0.037 *$ \\
\hline $\mathrm{C} 24$ & $0.30348(10)$ & $0.50564(10)$ & $0.49969(10)$ & $0.0245(3)$ \\
\hline $\mathrm{C} 25$ & $0.26657(10)$ & $0.57145(10)$ & $0.54301(10)$ & $0.0235(3)$ \\
\hline H25 & 0.247296 & 0.625774 & 0.508725 & $0.028 *$ \\
\hline $\mathrm{C} 26$ & $0.25718(9)$ & $0.55955(10)$ & $0.63571(10)$ & $0.0231(3)$ \\
\hline H26 & 0.231342 & 0.605643 & 0.663410 & $0.028 *$ \\
\hline $\mathrm{C} 27$ & 0.31087 (14) & $0.51780(13)$ & $0.39833(12)$ & $0.0414(4)$ \\
\hline $\mathrm{H} 27 \mathrm{~A}$ & 0.362859 & 0.485913 & 0.393138 & $0.062 *$ \\
\hline H27B & 0.316402 & 0.581651 & 0.385761 & $0.062^{*}$ \\
\hline
\end{tabular}




$\begin{array}{lllll}\text { H27C } & 0.258167 & 0.493772 & 0.350014 & 0.062^{*} \\ \text { C28 } & 0.00284(8) & 0.34193(8) & 1.11671(9) & 0.0179(2) \\ \text { C29 } & -0.03979(9) & 0.37551(9) & 1.18122(10) & 0.0215(2) \\ \text { H29 } & -0.096129 & 0.402330 & 1.155903 & 0.026^{*} \\ \text { C30 } & -0.00052(11) & 0.37002(9) & 1.28201(10) & 0.0257(3) \\ \text { H30 } & -0.031062 & 0.391957 & 1.324719 & 0.031^{*} \\ \text { C31 } & 0.08258(10) & 0.33305(9) & 1.32141(10) & 0.0254(3) \\ \text { C32 } & 0.12548(10) & 0.29963(9) & 1.25727(10) & 0.0238^{*}(3) \\ \text { H32 } & 0.182468 & 0.274300 & 1.282871 & 0.029^{*} \\ \text { C33 } & 0.08576(9) & 0.30297(9) & 1.15627(9) & 0.0202(2) \\ \text { H33 } & 0.115269 & 0.278520 & 1.113748 & 0.024^{*} \\ \text { C34 } & 0.12424(13) & 0.32882(12) & 1.43072(11) & 0.0371(4) \\ \text { H34A } & 0.148744 & 0.387517 & 1.454991 & 0.056^{*} \\ \text { H34B } & 0.171345 & 0.284084 & 1.446531 & 0.056^{*} \\ \text { H34C } & 0.079770 & 0.312095 & 1.461879 & 0.056^{*} \\ \text { C35 } & -0.22326(8) & 0.29502(8) & 0.78474(9) & 0.0181(2) \\ \text { C36 } & -0.22213(8) & 0.24553(9) & 0.70235(10) & 0.0205(2) \\ \text { H36 } & -0.167373 & 0.230297 & 0.693374 & 0.025^{*} \\ \text { C37 } & -0.29980(9) & 0.21818(10) & 0.63324(10) & 0.0235(3) \\ \text { H37 } & -0.297210 & 0.184450 & 0.577976 & 0.028^{*} \\ \text { C38 } & -0.38148(9) & 0.23948(10) & 0.64380(11) & 0.0250(3) \\ \text { C39 } & -0.38268(9) & 0.28783(10) & 0.72610(11) & 0.0263(3) \\ \text { H39 } & -0.437551 & 0.302475 & 0.735130 & 0.032^{*} \\ \text { C40 } & -0.30516(9) & 0.31540(10) & 0.79584(10) & 0.0232(3) \\ \text { H40 } & -0.307994 & 0.348378 & 0.851496 & 0.028^{*} \\ \text { C41 } & -0.46521(10) & 0.20943(12) & 0.56827(12) & 0.0343(3) \\ \text { H41A } & -0.480968 & 0.149536 & 0.585162 & 0.051^{*} \\ \text { H41B } & -0.456395 & 0.207820 & 0.503525 & 0.051^{*} \\ \text { H41C } & -0.512642 & 0.251416 & 0.567071 & 0.051^{*} \\ & & & & \end{array}$

Atomic displacement parameters $\left(\AA^{2}\right)$

\begin{tabular}{lllllll}
\hline & $U^{11}$ & $U^{22}$ & $U^{33}$ & $U^{12}$ & $U^{13}$ & $U^{23}$ \\
\hline $\mathrm{Ni}$ & $0.01402(8)$ & $0.01717(8)$ & $0.01271(8)$ & $-0.00050(5)$ & $0.00565(6)$ & $0.00038(5)$ \\
$\mathrm{N} 1$ & $0.0149(5)$ & $0.0218(5)$ & $0.0145(4)$ & $-0.0004(4)$ & $0.0055(4)$ & $0.0009(4)$ \\
$\mathrm{N} 2$ & $0.0152(4)$ & $0.0168(5)$ & $0.0146(4)$ & $0.0003(4)$ & $0.0063(4)$ & $0.0006(3)$ \\
$\mathrm{N} 3$ & $0.0164(5)$ & $0.0190(5)$ & $0.0145(4)$ & $-0.0014(4)$ & $0.0062(4)$ & $0.0005(4)$ \\
$\mathrm{N} 4$ & $0.0192(5)$ & $0.0189(5)$ & $0.0165(5)$ & $-0.0006(4)$ & $0.0070(4)$ & $-0.0003(4)$ \\
$\mathrm{C} 1$ & $0.0156(5)$ & $0.0250(6)$ & $0.0168(5)$ & $-0.0010(4)$ & $0.0055(4)$ & $0.0006(5)$ \\
$\mathrm{C} 2$ & $0.0157(5)$ & $0.0339(7)$ & $0.0198(6)$ & $-0.0026(5)$ & $0.0074(5)$ & $0.0014(5)$ \\
$\mathrm{C} 3$ & $0.0178(5)$ & $0.0237(6)$ & $0.0167(5)$ & $-0.0007(4)$ & $0.0079(4)$ & $0.0011(4)$ \\
$\mathrm{C} 4$ & $0.0168(5)$ & $0.0205(6)$ & $0.0151(5)$ & $-0.0002(4)$ & $0.0064(4)$ & $0.0011(4)$ \\
$\mathrm{C} 5$ & $0.0169(5)$ & $0.0171(5)$ & $0.0161(5)$ & $0.0006(4)$ & $0.0071(4)$ & $0.0001(4)$ \\
$\mathrm{C} 6$ & $0.0214(6)$ & $0.0206(6)$ & $0.0151(5)$ & $-0.0011(4)$ & $0.0080(4)$ & $-0.0001(4)$ \\
$\mathrm{C} 7$ & $0.0216(6)$ & $0.0235(6)$ & $0.0144(5)$ & $-0.0015(5)$ & $0.0049(5)$ & $-0.0003(4)$ \\
$\mathrm{C} 8$ & $0.0172(5)$ & $0.0227(6)$ & $0.0166(5)$ & $-0.0015(4)$ & $0.0045(4)$ & $-0.0003(4)$ \\
$\mathrm{C} 9$ & $0.0161(5)$ & $0.0158(5)$ & $0.0167(5)$ & $0.0002(4)$ & $0.0065(4)$ & $-0.0004(4)$ \\
$\mathrm{C} 10$ & $0.0161(5)$ & $0.0179(5)$ & $0.0154(5)$ & $-0.0003(4)$ & $0.0061(4)$ & $-0.0003(4)$
\end{tabular}




\begin{tabular}{|c|c|c|c|c|c|c|}
\hline C11 & $0.0165(5)$ & $0.0162(5)$ & $0.0195(5)$ & -0.0009 (4) & $0.0072(4)$ & $-0.0010(4)$ \\
\hline $\mathrm{C} 12$ & $0.0196(6)$ & $0.0191(6)$ & $0.0198(5)$ & $-0.0020(4)$ & $0.0099(5)$ & 0.0007 (4) \\
\hline $\mathrm{C} 13$ & $0.0202(5)$ & $0.0169(5)$ & $0.0168(5)$ & $-0.0004(4)$ & 0.0089 (4) & $0.0006(4)$ \\
\hline $\mathrm{C} 14$ & $0.0145(5)$ & $0.0274(6)$ & $0.0175(5)$ & $0.0028(5)$ & 0.0057 (4) & $0.0004(5)$ \\
\hline $\mathrm{C} 15$ & 0.0199 (6) & $0.0261(7)$ & $0.0210(6)$ & $0.0009(5)$ & $0.0056(5)$ & $0.0010(5)$ \\
\hline $\mathrm{C} 16$ & $0.0259(7)$ & $0.0308(7)$ & $0.0224(6)$ & $0.0046(5)$ & $0.0074(5)$ & $0.0052(5)$ \\
\hline $\mathrm{C} 17$ & $0.0211(6)$ & $0.0423(9)$ & $0.0177(6)$ & $0.0060(6)$ & $0.0047(5)$ & $-0.0007(5)$ \\
\hline C18 & $0.0181(6)$ & $0.0398(8)$ & $0.0230(6)$ & $-0.0020(5)$ & $0.0044(5)$ & $-0.0058(6)$ \\
\hline C19 & $0.0179(6)$ & $0.0303(7)$ & $0.0222(6)$ & $-0.0013(5)$ & $0.0071(5)$ & $-0.0014(5)$ \\
\hline $\mathrm{C} 20$ & $0.0319(8)$ & $0.0580(11)$ & $0.0229(7)$ & $0.0069(8)$ & $0.0007(6)$ & $0.0023(7)$ \\
\hline $\mathrm{C} 21$ & $0.0162(5)$ & $0.0238(6)$ & $0.0173(5)$ & $-0.0025(4)$ & $0.0075(4)$ & 0.0002 (4) \\
\hline $\mathrm{C} 22$ & $0.0366(8)$ & $0.0233(7)$ & $0.0228(6)$ & $0.0056(5)$ & $0.0137(6)$ & $0.0039(5)$ \\
\hline $\mathrm{C} 23$ & $0.0443(9)$ & $0.0289(7)$ & $0.0246(7)$ & $0.0100(6)$ & $0.0186(6)$ & $0.0018(5)$ \\
\hline $\mathrm{C} 24$ & $0.0297(7)$ & $0.0277(7)$ & $0.0194(6)$ & $0.0019(5)$ & $0.0122(5)$ & $0.0017(5)$ \\
\hline $\mathrm{C} 25$ & $0.0269(7)$ & $0.0246(6)$ & $0.0199(6)$ & $0.0028(5)$ & $0.0082(5)$ & $0.0038(5)$ \\
\hline C26 & $0.0253(6)$ & $0.0255(6)$ & $0.0211(6)$ & $0.0049(5)$ & $0.0107(5)$ & $0.0012(5)$ \\
\hline $\mathrm{C} 27$ & $0.0639(12)$ & $0.0437(10)$ & $0.0251(7)$ & $0.0160(9)$ & $0.0263(8)$ & $0.0079(7)$ \\
\hline $\mathrm{C} 28$ & $0.0229(6)$ & $0.0163(5)$ & $0.0165(5)$ & -0.0031 & $0.0089(5)$ & 0.0011 (4) \\
\hline $\mathrm{C} 29$ & $0.0277(6)$ & $0.0182(6)$ & $0.0220(6)$ & $-0.0020(5)$ & $0.0126(5)$ & $0.0005(5)$ \\
\hline C30 & $0.0409(8)$ & $0.0204(6)$ & $0.0208(6)$ & $-0.0034(5)$ & $0.0169(6)$ & $-0.0027(5)$ \\
\hline C31 & $0.0396(8)$ & $0.0198(6)$ & $0.0168(6)$ & $-0.0074(5)$ & $0.0085(5)$ & $0.0002(5)$ \\
\hline $\mathrm{C} 32$ & $0.0269(6)$ & $0.0232(6)$ & $0.0193(6)$ & $-0.0029(5)$ & $0.0042(5)$ & $0.0026(5)$ \\
\hline $\mathrm{C} 33$ & $0.0246(6)$ & $0.0194(6)$ & $0.0182(5)$ & $-0.0022(5)$ & $0.0091(5)$ & 0.0015 (4) \\
\hline $\mathrm{C} 34$ & $0.0552(10)$ & $0.0357(8)$ & $0.0172(6)$ & $-0.0063(7)$ & $0.0062(7)$ & $0.0004(6)$ \\
\hline $\mathrm{C} 35$ & $0.0162(5)$ & $0.0183(6)$ & $0.0210(6)$ & -0.0011 & $0.0074(4)$ & $0.0016(4)$ \\
\hline $\mathrm{C} 36$ & $0.0172(5)$ & $0.0216(6)$ & $0.0239(6)$ & -0.0020 & $0.0080(5)$ & $-0.0016(5)$ \\
\hline $\mathrm{C} 37$ & $0.0227(6)$ & $0.0250(6)$ & $0.0228(6)$ & $-0.0056(5)$ & $0.0070(5)$ & $-0.0015(5)$ \\
\hline C38 & $0.0183(6)$ & $0.0282(7)$ & $0.0270(6)$ & -0.0067 (5) & $0.0049(5)$ & $0.0054(5)$ \\
\hline C39 & $0.0160(6)$ & $0.0327(7)$ & $0.0319(7)$ & $-0.0012(5)$ & $0.0099(5)$ & $0.0030(6)$ \\
\hline $\mathrm{C} 40$ & $0.0192(6)$ & $0.0274(7)$ & $0.0264(6)$ & $-0.0011(5)$ & $0.0120(5)$ & $-0.0003(5)$ \\
\hline C41 & $0.0215(7)$ & $0.0433(9)$ & $0.0332(8)$ & $-0.0101(6)$ & $0.0011(6)$ & $0.0045(7)$ \\
\hline
\end{tabular}

Geometric parameters $\left(\AA,{ }^{\circ}\right)$

\begin{tabular}{llll}
\hline $\mathrm{Ni}-\mathrm{N} 1$ & $1.8858(10)$ & $\mathrm{C} 20-\mathrm{H} 20 \mathrm{~A}$ & 0.9800 \\
$\mathrm{Ni}-\mathrm{N} 2$ & $1.8490(10)$ & $\mathrm{C} 20-\mathrm{H} 20 \mathrm{~B}$ & 0.9800 \\
$\mathrm{Ni}-\mathrm{N} 3$ & $1.8876(10)$ & $\mathrm{C} 20-\mathrm{H} 20 \mathrm{C}$ & 0.9800 \\
$\mathrm{Ni}-\mathrm{N} 4$ & $1.9291(11)$ & $\mathrm{C} 21-\mathrm{C} 22$ & $1.3917(19)$ \\
$\mathrm{N} 1-\mathrm{C} 1$ & $1.3679(16)$ & $\mathrm{C} 21-\mathrm{C} 26$ & $1.3932(19)$ \\
$\mathrm{N} 1-\mathrm{C} 4$ & $1.3871(15)$ & $\mathrm{C} 22-\mathrm{H} 22$ & 0.9500 \\
$\mathrm{~N} 2-\mathrm{C} 5$ & $1.3554(15)$ & $\mathrm{C} 22-\mathrm{C} 23$ & $1.394(2)$ \\
$\mathrm{N} 2-\mathrm{C} 9$ & $1.3558(16)$ & $\mathrm{C} 23-\mathrm{H} 23$ & 0.9500 \\
$\mathrm{~N} 3-\mathrm{C} 10$ & $1.3920(15)$ & $\mathrm{C} 23-\mathrm{C} 24$ & $1.391(2)$ \\
$\mathrm{N} 3-\mathrm{C} 13$ & $1.3655(15)$ & $\mathrm{C} 24-\mathrm{C} 25$ & $1.3871(19)$ \\
$\mathrm{N} 4-\mathrm{H} 4 \mathrm{~A}$ & $0.89(3)$ & $\mathrm{C} 24-\mathrm{C} 27$ & $1.5033(19)$ \\
$\mathrm{N} 4-\mathrm{H} 4 \mathrm{~B}$ & $0.84(3)$ & $\mathrm{C} 25-\mathrm{H} 25$ & 0.9500 \\
$\mathrm{~N} 4-\mathrm{H} 4 \mathrm{C}$ & $0.86(3)$ & $\mathrm{C} 25-\mathrm{C} 26$ & $1.3925(18)$ \\
$\mathrm{C} 1-\mathrm{C} 2$ & $1.3996(17)$ & $\mathrm{C} 26-\mathrm{H} 26$ & 0.9500
\end{tabular}




\begin{tabular}{|c|c|c|c|}
\hline $\mathrm{C} 1-\mathrm{C} 14$ & $1.4699(17)$ & $\mathrm{C} 27-\mathrm{H} 27 \mathrm{~A}$ & 0.9800 \\
\hline $\mathrm{C} 2-\mathrm{H} 2$ & 0.9500 & $\mathrm{C} 27-\mathrm{H} 27 \mathrm{~B}$ & 0.9800 \\
\hline $\mathrm{C} 2-\mathrm{C} 3$ & $1.4080(18)$ & $\mathrm{C} 27-\mathrm{H} 27 \mathrm{C}$ & 0.9800 \\
\hline $\mathrm{C} 3-\mathrm{C} 4$ & $1.3968(17)$ & $\mathrm{C} 28-\mathrm{C} 29$ & $1.3981(17)$ \\
\hline $\mathrm{C} 3-\mathrm{C} 21$ & $1.4818(17)$ & $\mathrm{C} 28-\mathrm{C} 33$ & $1.3986(19)$ \\
\hline $\mathrm{C} 4-\mathrm{C} 5$ & $1.4400(17)$ & $\mathrm{C} 29-\mathrm{H} 29$ & 0.9500 \\
\hline $\mathrm{C} 5-\mathrm{C} 6$ & $1.3986(17)$ & $\mathrm{C} 29-\mathrm{C} 30$ & $1.3894(19)$ \\
\hline $\mathrm{C} 6-\mathrm{H} 6$ & 0.9500 & $\mathrm{C} 30-\mathrm{H} 30$ & 0.9500 \\
\hline $\mathrm{C} 6-\mathrm{C} 7$ & $1.3891(18)$ & $\mathrm{C} 30-\mathrm{C} 31$ & $1.389(2)$ \\
\hline $\mathrm{C} 7-\mathrm{H} 7$ & 0.9500 & $\mathrm{C} 31-\mathrm{C} 32$ & $1.396(2)$ \\
\hline $\mathrm{C} 7-\mathrm{C} 8$ & $1.3930(18)$ & $\mathrm{C} 31-\mathrm{C} 34$ & $1.5036(19)$ \\
\hline $\mathrm{C} 8-\mathrm{H} 8$ & 0.9500 & $\mathrm{C} 32-\mathrm{H} 32$ & 0.9500 \\
\hline $\mathrm{C} 8-\mathrm{C} 9$ & $1.3957(17)$ & $\mathrm{C} 32-\mathrm{C} 33$ & $1.3915(18)$ \\
\hline $\mathrm{C} 9-\mathrm{C} 10$ & $1.4511(16)$ & C33-H33 & 0.9500 \\
\hline $\mathrm{C} 10-\mathrm{C} 11$ & $1.4040(16)$ & $\mathrm{C} 34-\mathrm{H} 34 \mathrm{~A}$ & 0.9800 \\
\hline $\mathrm{C} 11-\mathrm{C} 12$ & $1.4151(17)$ & C34-H34B & 0.9800 \\
\hline $\mathrm{C} 11-\mathrm{C} 35$ & $1.4733(17)$ & $\mathrm{C} 34-\mathrm{H} 34 \mathrm{C}$ & 0.9800 \\
\hline $\mathrm{C} 12-\mathrm{H} 12$ & 0.9500 & $\mathrm{C} 35-\mathrm{C} 36$ & $1.3976(18)$ \\
\hline $\mathrm{C} 12-\mathrm{C} 13$ & $1.3992(17)$ & $\mathrm{C} 35-\mathrm{C} 40$ & $1.3993(17)$ \\
\hline $\mathrm{C} 13-\mathrm{C} 28$ & $1.4702(17)$ & $\mathrm{C} 36-\mathrm{H} 36$ & 0.9500 \\
\hline $\mathrm{C} 14-\mathrm{C} 15$ & $1.4020(19)$ & $\mathrm{C} 36-\mathrm{C} 37$ & $1.3912(18)$ \\
\hline $\mathrm{C} 14-\mathrm{C} 19$ & $1.3976(19)$ & C37-H37 & 0.9500 \\
\hline C15-H15 & 0.9500 & $\mathrm{C} 37-\mathrm{C} 38$ & $1.396(2)$ \\
\hline $\mathrm{C} 15-\mathrm{C} 16$ & $1.3907(19)$ & C38-C39 & $1.388(2)$ \\
\hline $\mathrm{C} 16-\mathrm{H} 16$ & 0.9500 & $\mathrm{C} 38-\mathrm{C} 41$ & $1.509(2)$ \\
\hline $\mathrm{C} 16-\mathrm{C} 17$ & $1.393(2)$ & C39-H39 & 0.9500 \\
\hline $\mathrm{C} 17-\mathrm{C} 18$ & $1.390(2)$ & $\mathrm{C} 39-\mathrm{C} 40$ & $1.3945(19)$ \\
\hline $\mathrm{C} 17-\mathrm{C} 20$ & $1.509(2)$ & $\mathrm{C} 40-\mathrm{H} 40$ & 0.9500 \\
\hline $\mathrm{C} 18-\mathrm{H} 18$ & 0.9500 & $\mathrm{C} 41-\mathrm{H} 41 \mathrm{~A}$ & 0.9800 \\
\hline $\mathrm{C} 18-\mathrm{C} 19$ & $1.3929(19)$ & $\mathrm{C} 41-\mathrm{H} 41 \mathrm{~B}$ & 0.9800 \\
\hline C19-H19 & 0.9500 & $\mathrm{C} 41-\mathrm{H} 41 \mathrm{C}$ & 0.9800 \\
\hline $\mathrm{N} 1-\mathrm{Ni}-\mathrm{N} 3$ & $163.95(5)$ & $\mathrm{C} 17-\mathrm{C} 20-\mathrm{H} 20 \mathrm{~A}$ & 109.5 \\
\hline $\mathrm{N} 1-\mathrm{Ni}-\mathrm{N} 4$ & $96.98(5)$ & $\mathrm{C} 17-\mathrm{C} 20-\mathrm{H} 20 \mathrm{~B}$ & 109.5 \\
\hline $\mathrm{N} 2-\mathrm{Ni}-\mathrm{N} 1$ & $83.41(4)$ & $\mathrm{C} 17-\mathrm{C} 20-\mathrm{H} 20 \mathrm{C}$ & 109.5 \\
\hline $\mathrm{N} 2-\mathrm{Ni}-\mathrm{N} 3$ & $82.95(4)$ & $\mathrm{H} 20 \mathrm{~A}-\mathrm{C} 20-\mathrm{H} 20 \mathrm{~B}$ & 109.5 \\
\hline $\mathrm{N} 2-\mathrm{Ni}-\mathrm{N} 4$ & $162.16(5)$ & $\mathrm{H} 20 \mathrm{~A}-\mathrm{C} 20-\mathrm{H} 20 \mathrm{C}$ & 109.5 \\
\hline $\mathrm{N} 3-\mathrm{Ni}-\mathrm{N} 4$ & $98.59(5)$ & $\mathrm{H} 20 \mathrm{~B}-\mathrm{C} 20-\mathrm{H} 20 \mathrm{C}$ & 109.5 \\
\hline $\mathrm{C} 1-\mathrm{N} 1-\mathrm{Ni}$ & $140.10(9)$ & $\mathrm{C} 22-\mathrm{C} 21-\mathrm{C} 3$ & $120.99(12)$ \\
\hline $\mathrm{C} 1-\mathrm{N} 1-\mathrm{C} 4$ & $106.75(10)$ & $\mathrm{C} 22-\mathrm{C} 21-\mathrm{C} 26$ & $117.86(12)$ \\
\hline $\mathrm{C} 4-\mathrm{N} 1-\mathrm{Ni}$ & $113.07(8)$ & $\mathrm{C} 26-\mathrm{C} 21-\mathrm{C} 3$ & $121.15(12)$ \\
\hline $\mathrm{C} 5-\mathrm{N} 2-\mathrm{Ni}$ & $117.40(8)$ & $\mathrm{C} 21-\mathrm{C} 22-\mathrm{H} 22$ & 119.6 \\
\hline $\mathrm{C} 5-\mathrm{N} 2-\mathrm{C} 9$ & $123.28(11)$ & $\mathrm{C} 21-\mathrm{C} 22-\mathrm{C} 23$ & $120.86(13)$ \\
\hline $\mathrm{C} 9-\mathrm{N} 2-\mathrm{Ni}$ & $118.43(8)$ & $\mathrm{C} 23-\mathrm{C} 22-\mathrm{H} 22$ & 119.6 \\
\hline $\mathrm{C} 10-\mathrm{N} 3-\mathrm{Ni}$ & $114.29(8)$ & $\mathrm{C} 22-\mathrm{C} 23-\mathrm{H} 23$ & 119.4 \\
\hline $\mathrm{C} 13-\mathrm{N} 3-\mathrm{Ni}$ & $138.51(9)$ & $\mathrm{C} 24-\mathrm{C} 23-\mathrm{C} 22$ & $121.30(13)$ \\
\hline $\mathrm{C} 13-\mathrm{N} 3-\mathrm{C} 10$ & $107.19(10)$ & $\mathrm{C} 24-\mathrm{C} 23-\mathrm{H} 23$ & 119.4 \\
\hline $\mathrm{Ni}-\mathrm{N} 4-\mathrm{H} 4 \mathrm{~A}$ & $113.0(16)$ & $\mathrm{C} 23-\mathrm{C} 24-\mathrm{C} 27$ & $121.29(13)$ \\
\hline
\end{tabular}




\begin{tabular}{|c|c|}
\hline $\mathrm{Ni}-\mathrm{N} 4-\mathrm{H} 4 \mathrm{~B}$ & $120.3(18)$ \\
\hline $\mathrm{Ni}-\mathrm{N} 4-\mathrm{H} 4 \mathrm{C}$ & $106.2(16)$ \\
\hline $\mathrm{H} 4 \mathrm{~A}-\mathrm{N} 4-\mathrm{H} 4 \mathrm{~B}$ & $103(2)$ \\
\hline $\mathrm{H} 4 \mathrm{~A}-\mathrm{N} 4-\mathrm{H} 4 \mathrm{C}$ & $111(2)$ \\
\hline $\mathrm{H} 4 \mathrm{~B}-\mathrm{N} 4-\mathrm{H} 4 \mathrm{C}$ & $103(2)$ \\
\hline $\mathrm{N} 1-\mathrm{C} 1-\mathrm{C} 2$ & $109.43(11)$ \\
\hline $\mathrm{N} 1-\mathrm{C} 1-\mathrm{C} 14$ & $123.64(11)$ \\
\hline $\mathrm{C} 2-\mathrm{C} 1-\mathrm{C} 14$ & $126.55(11)$ \\
\hline $\mathrm{C} 1-\mathrm{C} 2-\mathrm{H} 2$ & 126.1 \\
\hline $\mathrm{C} 1-\mathrm{C} 2-\mathrm{C} 3$ & $107.83(11)$ \\
\hline $\mathrm{C} 3-\mathrm{C} 2-\mathrm{H} 2$ & 126.1 \\
\hline $\mathrm{C} 2-\mathrm{C} 3-\mathrm{C} 21$ & $127.47(11)$ \\
\hline $\mathrm{C} 4-\mathrm{C} 3-\mathrm{C} 2$ & $105.55(11)$ \\
\hline $\mathrm{C} 4-\mathrm{C} 3-\mathrm{C} 21$ & $126.95(11)$ \\
\hline $\mathrm{N} 1-\mathrm{C} 4-\mathrm{C} 3$ & $110.43(11)$ \\
\hline $\mathrm{N} 1-\mathrm{C} 4-\mathrm{C} 5$ & $113.98(10)$ \\
\hline $\mathrm{C} 3-\mathrm{C} 4-\mathrm{C} 5$ & $135.45(11)$ \\
\hline $\mathrm{N} 2-\mathrm{C} 5-\mathrm{C} 4$ & $110.79(11)$ \\
\hline $\mathrm{N} 2-\mathrm{C} 5-\mathrm{C} 6$ & $119.41(11)$ \\
\hline $\mathrm{C} 6-\mathrm{C} 5-\mathrm{C} 4$ & $129.80(11)$ \\
\hline $\mathrm{C} 5-\mathrm{C} 6-\mathrm{H} 6$ & 120.9 \\
\hline $\mathrm{C} 7-\mathrm{C} 6-\mathrm{C} 5$ & $118.23(11)$ \\
\hline $\mathrm{C} 7-\mathrm{C} 6-\mathrm{H} 6$ & 120.9 \\
\hline $\mathrm{C} 6-\mathrm{C} 7-\mathrm{H} 7$ & 119.3 \\
\hline $\mathrm{C} 6-\mathrm{C} 7-\mathrm{C} 8$ & $121.36(12)$ \\
\hline $\mathrm{C} 8-\mathrm{C} 7-\mathrm{H} 7$ & 119.3 \\
\hline $\mathrm{C} 7-\mathrm{C} 8-\mathrm{H} 8$ & 120.6 \\
\hline $\mathrm{C} 7-\mathrm{C} 8-\mathrm{C} 9$ & $118.76(12)$ \\
\hline $\mathrm{C} 9-\mathrm{C} 8-\mathrm{H} 8$ & 120.6 \\
\hline $\mathrm{N} 2-\mathrm{C} 9-\mathrm{C} 8$ & $118.92(11)$ \\
\hline $\mathrm{N} 2-\mathrm{C} 9-\mathrm{C} 10$ & $110.48(10)$ \\
\hline $\mathrm{C} 8-\mathrm{C} 9-\mathrm{C} 10$ & $130.58(11)$ \\
\hline $\mathrm{N} 3-\mathrm{C} 10-\mathrm{C} 9$ & $113.09(10)$ \\
\hline $\mathrm{N} 3-\mathrm{C} 10-\mathrm{C} 11$ & $109.98(10)$ \\
\hline $\mathrm{C} 11-\mathrm{C} 10-\mathrm{C} 9$ & $136.93(11)$ \\
\hline $\mathrm{C} 10-\mathrm{C} 11-\mathrm{C} 12$ & $105.46(11)$ \\
\hline $\mathrm{C} 10-\mathrm{C} 11-\mathrm{C} 35$ & $130.14(11)$ \\
\hline $\mathrm{C} 12-\mathrm{C} 11-\mathrm{C} 35$ & $124.37(11)$ \\
\hline $\mathrm{C} 11-\mathrm{C} 12-\mathrm{H} 12$ & 126.1 \\
\hline $\mathrm{C} 13-\mathrm{C} 12-\mathrm{C} 11$ & $107.90(11)$ \\
\hline $\mathrm{C} 13-\mathrm{C} 12-\mathrm{H} 12$ & 126.1 \\
\hline $\mathrm{N} 3-\mathrm{C} 13-\mathrm{C} 12$ & $109.47(11)$ \\
\hline $\mathrm{N} 3-\mathrm{C} 13-\mathrm{C} 28$ & $123.82(11)$ \\
\hline $\mathrm{C} 12-\mathrm{C} 13-\mathrm{C} 28$ & $126.61(11)$ \\
\hline $\mathrm{C} 15-\mathrm{C} 14-\mathrm{C} 1$ & $121.12(12)$ \\
\hline $\mathrm{C} 19-\mathrm{C} 14-\mathrm{C} 1$ & $120.80(12)$ \\
\hline $\mathrm{C} 19-\mathrm{C} 14-\mathrm{C} 15$ & $117.94(12)$ \\
\hline $\mathrm{C} 14-\mathrm{C} 15-\mathrm{H} 15$ & 119.6 \\
\hline
\end{tabular}

\begin{tabular}{|c|c|}
\hline $\mathrm{C} 25-\mathrm{C} 24-\mathrm{C} 23$ & $117.64(12)$ \\
\hline $\mathrm{C} 25-\mathrm{C} 24-\mathrm{C} 27$ & $121.07(13)$ \\
\hline $\mathrm{C} 24-\mathrm{C} 25-\mathrm{H} 25$ & 119.3 \\
\hline $\mathrm{C} 24-\mathrm{C} 25-\mathrm{C} 26$ & $121.37(13)$ \\
\hline $\mathrm{C} 26-\mathrm{C} 25-\mathrm{H} 25$ & 119.3 \\
\hline $\mathrm{C} 21-\mathrm{C} 26-\mathrm{H} 26$ & 119.5 \\
\hline $\mathrm{C} 25-\mathrm{C} 26-\mathrm{C} 21$ & $120.91(13)$ \\
\hline $\mathrm{C} 25-\mathrm{C} 26-\mathrm{H} 26$ & 119.5 \\
\hline $\mathrm{C} 24-\mathrm{C} 27-\mathrm{H} 27 \mathrm{~A}$ & 109.5 \\
\hline $\mathrm{C} 24-\mathrm{C} 27-\mathrm{H} 27 \mathrm{~B}$ & 109.5 \\
\hline $\mathrm{C} 24-\mathrm{C} 27-\mathrm{H} 27 \mathrm{C}$ & 109.5 \\
\hline $\mathrm{H} 27 \mathrm{~A}-\mathrm{C} 27-\mathrm{H} 27 \mathrm{~B}$ & 109.5 \\
\hline $\mathrm{H} 27 \mathrm{~A}-\mathrm{C} 27-\mathrm{H} 27 \mathrm{C}$ & 109.5 \\
\hline $\mathrm{H} 27 \mathrm{~B}-\mathrm{C} 27-\mathrm{H} 27 \mathrm{C}$ & 109.5 \\
\hline $\mathrm{C} 29-\mathrm{C} 28-\mathrm{C} 13$ & $119.81(12)$ \\
\hline $\mathrm{C} 29-\mathrm{C} 28-\mathrm{C} 33$ & $118.31(12)$ \\
\hline $\mathrm{C} 33-\mathrm{C} 28-\mathrm{C} 13$ & $121.79(11)$ \\
\hline $\mathrm{C} 28-\mathrm{C} 29-\mathrm{H} 29$ & 119.7 \\
\hline $\mathrm{C} 30-\mathrm{C} 29-\mathrm{C} 28$ & $120.60(13)$ \\
\hline $\mathrm{C} 30-\mathrm{C} 29-\mathrm{H} 29$ & 119.7 \\
\hline $\mathrm{C} 29-\mathrm{C} 30-\mathrm{H} 30$ & 119.4 \\
\hline $\mathrm{C} 31-\mathrm{C} 30-\mathrm{C} 29$ & $121.17(13)$ \\
\hline $\mathrm{C} 31-\mathrm{C} 30-\mathrm{H} 30$ & 119.4 \\
\hline $\mathrm{C} 30-\mathrm{C} 31-\mathrm{C} 32$ & $118.41(12)$ \\
\hline $\mathrm{C} 30-\mathrm{C} 31-\mathrm{C} 34$ & $120.24(14)$ \\
\hline $\mathrm{C} 32-\mathrm{C} 31-\mathrm{C} 34$ & $121.35(15)$ \\
\hline $\mathrm{C} 31-\mathrm{C} 32-\mathrm{H} 32$ & 119.6 \\
\hline $\mathrm{C} 33-\mathrm{C} 32-\mathrm{C} 31$ & $120.78(13)$ \\
\hline $\mathrm{C} 33-\mathrm{C} 32-\mathrm{H} 32$ & 119.6 \\
\hline $\mathrm{C} 28-\mathrm{C} 33-\mathrm{H} 33$ & 119.7 \\
\hline $\mathrm{C} 32-\mathrm{C} 33-\mathrm{C} 28$ & $120.69(12)$ \\
\hline $\mathrm{C} 32-\mathrm{C} 33-\mathrm{H} 33$ & 119.7 \\
\hline $\mathrm{C} 31-\mathrm{C} 34-\mathrm{H} 34 \mathrm{~A}$ & 109.5 \\
\hline $\mathrm{C} 31-\mathrm{C} 34-\mathrm{H} 34 \mathrm{~B}$ & 109.5 \\
\hline $\mathrm{C} 31-\mathrm{C} 34-\mathrm{H} 34 \mathrm{C}$ & 109.5 \\
\hline $\mathrm{H} 34 \mathrm{~A}-\mathrm{C} 34-\mathrm{H} 34 \mathrm{~B}$ & 109.5 \\
\hline $\mathrm{H} 34 \mathrm{~A}-\mathrm{C} 34-\mathrm{H} 34 \mathrm{C}$ & 109.5 \\
\hline $\mathrm{H} 34 \mathrm{~B}-\mathrm{C} 34-\mathrm{H} 34 \mathrm{C}$ & 109.5 \\
\hline $\mathrm{C} 36-\mathrm{C} 35-\mathrm{C} 11$ & $121.84(11)$ \\
\hline $\mathrm{C} 36-\mathrm{C} 35-\mathrm{C} 40$ & $117.73(12)$ \\
\hline $\mathrm{C} 40-\mathrm{C} 35-\mathrm{C} 11$ & $120.38(12)$ \\
\hline $\mathrm{C} 35-\mathrm{C} 36-\mathrm{H} 36$ & 119.4 \\
\hline $\mathrm{C} 37-\mathrm{C} 36-\mathrm{C} 35$ & $121.14(12)$ \\
\hline $\mathrm{C} 37-\mathrm{C} 36-\mathrm{H} 36$ & 119.4 \\
\hline $\mathrm{C} 36-\mathrm{C} 37-\mathrm{H} 37$ & 119.5 \\
\hline $\mathrm{C} 36-\mathrm{C} 37-\mathrm{C} 38$ & $121.07(13)$ \\
\hline $\mathrm{C} 38-\mathrm{C} 37-\mathrm{H} 37$ & 119.5 \\
\hline $\mathrm{C} 37-\mathrm{C} 38-\mathrm{C} 41$ & $120.51(14)$ \\
\hline
\end{tabular}




\begin{tabular}{|c|c|c|c|}
\hline $\mathrm{C} 16-\mathrm{C} 15-\mathrm{C} 14$ & $120.76(13)$ & $\mathrm{C} 39-\mathrm{C} 38-\mathrm{C} 37$ & $117.85(13)$ \\
\hline $\mathrm{C} 16-\mathrm{C} 15-\mathrm{H} 15$ & 119.6 & $\mathrm{C} 39-\mathrm{C} 38-\mathrm{C} 41$ & $121.63(14)$ \\
\hline $\mathrm{C} 15-\mathrm{C} 16-\mathrm{H} 16$ & 119.4 & $\mathrm{C} 38-\mathrm{C} 39-\mathrm{H} 39$ & 119.3 \\
\hline $\mathrm{C} 15-\mathrm{C} 16-\mathrm{C} 17$ & $121.11(14)$ & $\mathrm{C} 38-\mathrm{C} 39-\mathrm{C} 40$ & $121.48(13)$ \\
\hline $\mathrm{C} 17-\mathrm{C} 16-\mathrm{H} 16$ & 119.4 & $\mathrm{C} 40-\mathrm{C} 39-\mathrm{H} 39$ & 119.3 \\
\hline $\mathrm{C} 16-\mathrm{C} 17-\mathrm{C} 20$ & $120.48(15)$ & $\mathrm{C} 35-\mathrm{C} 40-\mathrm{H} 40$ & 119.6 \\
\hline $\mathrm{C} 18-\mathrm{C} 17-\mathrm{C} 16$ & $118.22(13)$ & $\mathrm{C} 39-\mathrm{C} 40-\mathrm{C} 35$ & $120.72(13)$ \\
\hline $\mathrm{C} 18-\mathrm{C} 17-\mathrm{C} 20$ & $121.26(15)$ & $\mathrm{C} 39-\mathrm{C} 40-\mathrm{H} 40$ & 119.6 \\
\hline $\mathrm{C} 17-\mathrm{C} 18-\mathrm{H} 18$ & 119.5 & $\mathrm{C} 38-\mathrm{C} 41-\mathrm{H} 41 \mathrm{~A}$ & 109.5 \\
\hline $\mathrm{C} 17-\mathrm{C} 18-\mathrm{C} 19$ & $121.09(14)$ & $\mathrm{C} 38-\mathrm{C} 41-\mathrm{H} 41 \mathrm{~B}$ & 109.5 \\
\hline $\mathrm{C} 19-\mathrm{C} 18-\mathrm{H} 18$ & 119.5 & $\mathrm{C} 38-\mathrm{C} 41-\mathrm{H} 41 \mathrm{C}$ & 109.5 \\
\hline $\mathrm{C} 14-\mathrm{C} 19-\mathrm{H} 19$ & 119.6 & $\mathrm{H} 41 \mathrm{~A}-\mathrm{C} 41-\mathrm{H} 41 \mathrm{~B}$ & 109.5 \\
\hline $\mathrm{C} 18-\mathrm{C} 19-\mathrm{C} 14$ & $120.85(14)$ & $\mathrm{H} 41 \mathrm{~A}-\mathrm{C} 41-\mathrm{H} 41 \mathrm{C}$ & 109.5 \\
\hline $\mathrm{C} 18-\mathrm{C} 19-\mathrm{H} 19$ & 119.6 & $\mathrm{H} 41 \mathrm{~B}-\mathrm{C} 41-\mathrm{H} 41 \mathrm{C}$ & 109.5 \\
\hline $\mathrm{Ni}-\mathrm{N} 1-\mathrm{C} 1-\mathrm{C} 2$ & $-175.37(11)$ & $\mathrm{C} 5-\mathrm{N} 2-\mathrm{C} 9-\mathrm{C} 10$ & $-178.59(11)$ \\
\hline $\mathrm{Ni}-\mathrm{N} 1-\mathrm{C} 1-\mathrm{C} 14$ & $11.3(2)$ & $\mathrm{C} 5-\mathrm{C} 6-\mathrm{C} 7-\mathrm{C} 8$ & $-0.50(19)$ \\
\hline $\mathrm{Ni}-\mathrm{N} 1-\mathrm{C} 4-\mathrm{C} 3$ & $176.50(9)$ & $\mathrm{C} 6-\mathrm{C} 7-\mathrm{C} 8-\mathrm{C} 9$ & $-1.2(2)$ \\
\hline $\mathrm{Ni}-\mathrm{N} 1-\mathrm{C} 4-\mathrm{C} 5$ & $-7.17(14)$ & $\mathrm{C} 7-\mathrm{C} 8-\mathrm{C} 9-\mathrm{N} 2$ & $1.34(18)$ \\
\hline $\mathrm{Ni}-\mathrm{N} 2-\mathrm{C} 5-\mathrm{C} 4$ & $9.41(13)$ & $\mathrm{C} 7-\mathrm{C} 8-\mathrm{C} 9-\mathrm{C} 10$ & $179.84(13)$ \\
\hline $\mathrm{Ni}-\mathrm{N} 2-\mathrm{C} 5-\mathrm{C} 6$ & $-170.85(9)$ & $\mathrm{C} 8-\mathrm{C} 9-\mathrm{C} 10-\mathrm{N} 3$ & $-172.66(13)$ \\
\hline $\mathrm{Ni}-\mathrm{N} 2-\mathrm{C} 9-\mathrm{C} 8$ & $169.03(9)$ & $\mathrm{C} 8-\mathrm{C} 9-\mathrm{C} 10-\mathrm{C} 11$ & $7.1(3)$ \\
\hline $\mathrm{Ni}-\mathrm{N} 2-\mathrm{C} 9-\mathrm{C} 10$ & $-9.75(14)$ & $\mathrm{C} 9-\mathrm{N} 2-\mathrm{C} 5-\mathrm{C} 4$ & $178.36(11)$ \\
\hline $\mathrm{Ni}-\mathrm{N} 3-\mathrm{C} 10-\mathrm{C} 9$ & $-0.02(13)$ & $\mathrm{C} 9-\mathrm{N} 2-\mathrm{C} 5-\mathrm{C} 6$ & $-1.90(18)$ \\
\hline $\mathrm{Ni}-\mathrm{N} 3-\mathrm{C} 10-\mathrm{C} 11$ & $-179.83(8)$ & $\mathrm{C} 9-\mathrm{C} 10-\mathrm{C} 11-\mathrm{C} 12$ & $-179.68(14)$ \\
\hline $\mathrm{Ni}-\mathrm{N} 3-\mathrm{C} 13-\mathrm{C} 12$ & $179.52(10)$ & $\mathrm{C} 9-\mathrm{C} 10-\mathrm{C} 11-\mathrm{C} 35$ & $2.3(2)$ \\
\hline $\mathrm{Ni}-\mathrm{N} 3-\mathrm{C} 13-\mathrm{C} 28$ & $-4.1(2)$ & $\mathrm{C} 10-\mathrm{N} 3-\mathrm{C} 13-\mathrm{C} 12$ & $-1.25(14)$ \\
\hline $\mathrm{N} 1-\mathrm{Ni}-\mathrm{N} 2-\mathrm{C} 5$ & $-10.93(9)$ & $\mathrm{C} 10-\mathrm{N} 3-\mathrm{C} 13-\mathrm{C} 28$ & $175.14(11)$ \\
\hline $\mathrm{N} 1-\mathrm{Ni}-\mathrm{N} 2-\mathrm{C} 9$ & $179.57(10)$ & $\mathrm{C} 10-\mathrm{C} 11-\mathrm{C} 12-\mathrm{C} 13$ & $-0.82(14)$ \\
\hline $\mathrm{N} 1-\mathrm{Ni}-\mathrm{N} 3-\mathrm{C} 10$ & $-36.0(2)$ & $\mathrm{C} 10-\mathrm{C} 11-\mathrm{C} 35-\mathrm{C} 36$ & $41.5(2)$ \\
\hline $\mathrm{N} 1-\mathrm{Ni}-\mathrm{N} 3-\mathrm{C} 13$ & $143.17(16)$ & $\mathrm{C} 10-\mathrm{C} 11-\mathrm{C} 35-\mathrm{C} 40$ & $-141.21(14)$ \\
\hline $\mathrm{N} 1-\mathrm{C} 1-\mathrm{C} 2-\mathrm{C} 3$ & $-0.70(16)$ & $\mathrm{C} 11-\mathrm{C} 12-\mathrm{C} 13-\mathrm{N} 3$ & $1.30(15)$ \\
\hline $\mathrm{N} 1-\mathrm{C} 1-\mathrm{C} 14-\mathrm{C} 15$ & $40.6(2)$ & $\mathrm{C} 11-\mathrm{C} 12-\mathrm{C} 13-\mathrm{C} 28$ & $-174.95(12)$ \\
\hline $\mathrm{N} 1-\mathrm{C} 1-\mathrm{C} 14-\mathrm{C} 19$ & $-143.73(13)$ & $\mathrm{C} 11-\mathrm{C} 35-\mathrm{C} 36-\mathrm{C} 37$ & $177.93(12)$ \\
\hline $\mathrm{N} 1-\mathrm{C} 4-\mathrm{C} 5-\mathrm{N} 2$ & $-1.13(15)$ & $\mathrm{C} 11-\mathrm{C} 35-\mathrm{C} 40-\mathrm{C} 39$ & $-178.08(13)$ \\
\hline $\mathrm{N} 1-\mathrm{C} 4-\mathrm{C} 5-\mathrm{C} 6$ & $179.17(12)$ & $\mathrm{C} 12-\mathrm{C} 11-\mathrm{C} 35-\mathrm{C} 36$ & $-136.15(14)$ \\
\hline $\mathrm{N} 2-\mathrm{Ni}-\mathrm{N} 1-\mathrm{C} 1$ & $-174.10(15)$ & $\mathrm{C} 12-\mathrm{C} 11-\mathrm{C} 35-\mathrm{C} 40$ & $41.12(19)$ \\
\hline $\mathrm{N} 2-\mathrm{Ni}-\mathrm{N} 1-\mathrm{C} 4$ & $9.65(9)$ & $\mathrm{C} 12-\mathrm{C} 13-\mathrm{C} 28-\mathrm{C} 29$ & $-47.47(19)$ \\
\hline $\mathrm{N} 2-\mathrm{Ni}-\mathrm{N} 3-\mathrm{C} 10$ & $-4.08(9)$ & $\mathrm{C} 12-\mathrm{C} 13-\mathrm{C} 28-\mathrm{C} 33$ & $129.04(14)$ \\
\hline $\mathrm{N} 2-\mathrm{Ni}-\mathrm{N} 3-\mathrm{C} 13$ & $175.12(14)$ & $\mathrm{C} 13-\mathrm{N} 3-\mathrm{C} 10-\mathrm{C} 9$ & $-179.46(10)$ \\
\hline $\mathrm{N} 2-\mathrm{C} 5-\mathrm{C} 6-\mathrm{C} 7$ & $2.00(18)$ & $\mathrm{C} 13-\mathrm{N} 3-\mathrm{C} 10-\mathrm{C} 11$ & $0.73(14)$ \\
\hline $\mathrm{N} 2-\mathrm{C} 9-\mathrm{C} 10-\mathrm{N} 3$ & $5.93(15)$ & $\mathrm{C} 13-\mathrm{C} 28-\mathrm{C} 29-\mathrm{C} 30$ & $176.54(12)$ \\
\hline $\mathrm{N} 2-\mathrm{C} 9-\mathrm{C} 10-\mathrm{C} 11$ & $-174.33(14)$ & $\mathrm{C} 13-\mathrm{C} 28-\mathrm{C} 33-\mathrm{C} 32$ & $-177.98(12)$ \\
\hline $\mathrm{N} 3-\mathrm{Ni}-\mathrm{N} 1-\mathrm{C} 1$ & $-142.19(16)$ & $\mathrm{C} 14-\mathrm{C} 1-\mathrm{C} 2-\mathrm{C} 3$ & $172.34(13)$ \\
\hline $\mathrm{N} 3-\mathrm{Ni}-\mathrm{N} 1-\mathrm{C} 4$ & $41.6(2)$ & $\mathrm{C} 14-\mathrm{C} 15-\mathrm{C} 16-\mathrm{C} 17$ & $-1.9(2)$ \\
\hline $\mathrm{N} 3-\mathrm{Ni}-\mathrm{N} 2-\mathrm{C} 5$ & $177.54(10)$ & $\mathrm{C} 15-\mathrm{C} 14-\mathrm{C} 19-\mathrm{C} 18$ & $0.7(2)$ \\
\hline $\mathrm{N} 3-\mathrm{Ni}-\mathrm{N} 2-\mathrm{C} 9$ & $8.03(9)$ & $\mathrm{C} 15-\mathrm{C} 16-\mathrm{C} 17-\mathrm{C} 18$ & $2.0(2)$ \\
\hline $\mathrm{N} 3-\mathrm{C} 10-\mathrm{C} 11-\mathrm{C} 12$ & $0.07(14)$ & $\mathrm{C} 15-\mathrm{C} 16-\mathrm{C} 17-\mathrm{C} 20$ & $-175.81(14)$ \\
\hline
\end{tabular}




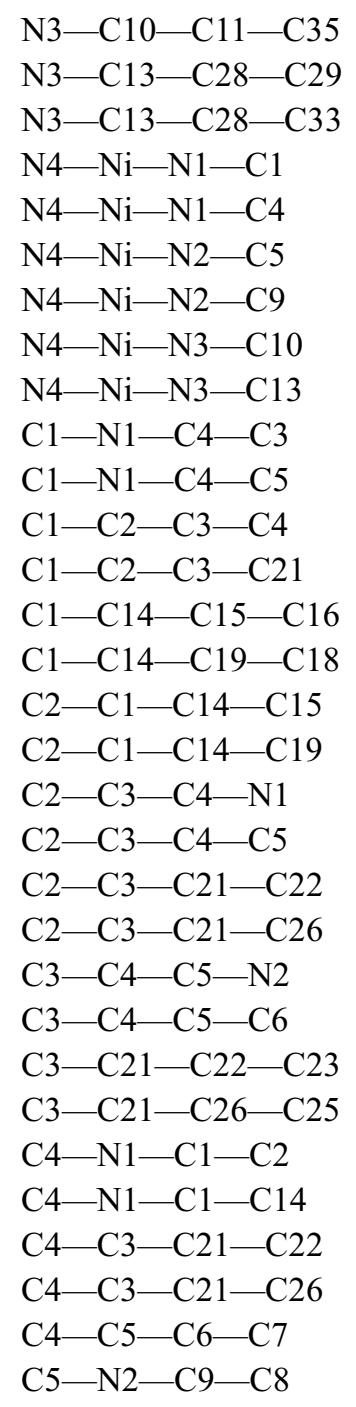

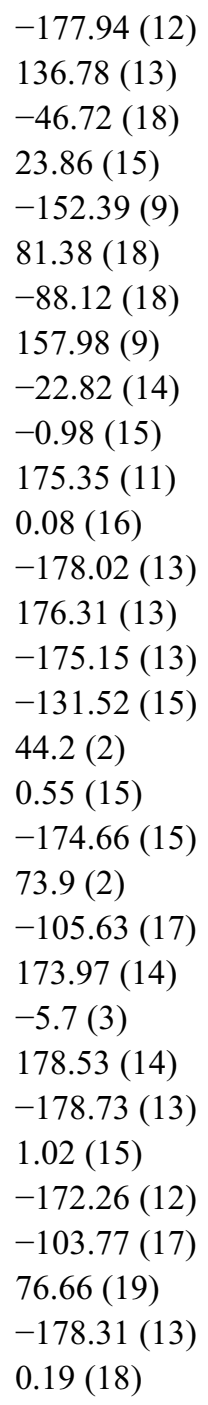

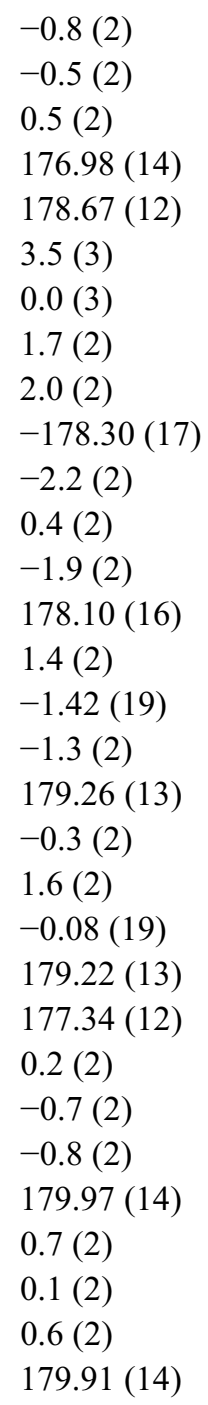

(Acetonitrile- $\kappa N)\left\{2,2^{\prime}\right.$-(pyridine-2,6-diyl)bis[3,5-bis(4-methylphenyl)pyrrolido- $\left.\left.\kappa N\right]\right\}$ nickel(II) (pyrr2PyNiCNMe)

\section{Crystal data}

$\left[\mathrm{Ni}\left(\mathrm{C}_{41} \mathrm{H}_{33} \mathrm{~N}_{3}\right)\left(\mathrm{C}_{2} \mathrm{H}_{3} \mathrm{~N}\right)\right]$

$M_{r}=753.64$

Triclinic, $P \overline{1}$

$a=11.2735(16) \AA$

$b=14.1802(19) \AA$

$c=14.688(2) \AA$

$\alpha=67.162(2)^{\circ}$

$\beta=68.881(2)^{\circ}$

$\gamma=80.665(2)^{\circ}$

$V=2018.0(5) \AA^{3}$
$Z=2$

$F(000)=800$

$D_{\mathrm{x}}=1.240 \mathrm{Mg} \mathrm{m}^{-3}$

Mo $K \alpha$ radiation, $\lambda=0.71073 \AA$

Cell parameters from 8799 reflections

$\theta=2.5-32.8^{\circ}$

$\mu=0.52 \mathrm{~mm}^{-1}$

$T=100 \mathrm{~K}$

Prism, colourless

$0.20 \times 0.12 \times 0.09 \mathrm{~mm}$ 


\author{
Data collection \\ Bruker APEXII CCD \\ 9994 independent reflections \\ diffractometer \\ Curved-graphite monochromator \\ 7327 reflections with $I>2 \sigma(I)$ \\ $\varphi$ and $\omega$ scans \\ Absorption correction: multi-scan \\ (SADABS; Bruker, 2016) \\ $T_{\min }=0.686, T_{\max }=0.899$ \\ 21869 measured reflections \\ $R_{\text {int }}=0.058$ \\ $\theta_{\text {max }}=28.3^{\circ}, \theta_{\min }=2.1^{\circ}$ \\ $h=-15 \rightarrow 15$ \\ $k=-18 \rightarrow 18$ \\ $l=-19 \rightarrow 19$

\section{Refinement} \\ Refinement on $F^{2}$ \\ Least-squares matrix: full \\ $R\left[F^{2}>2 \sigma\left(F^{2}\right)\right]=0.068$ \\ $w R\left(F^{2}\right)=0.193$ \\ $S=1.00$ \\ 9994 reflections \\ 551 parameters \\ 178 restraints \\ Primary atom site location: dual \\ Secondary atom site location: difference Fourier \\ map \\ Hydrogen site location: inferred from \\ neighbouring sites \\ $\mathrm{H}$-atom parameters constrained \\ $w=1 /\left[\sigma^{2}\left(F_{\mathrm{o}}^{2}\right)+(0.1214 P)^{2}\right]$ \\ where $P=\left(F_{\mathrm{o}}^{2}+2 F_{\mathrm{c}}^{2}\right) / 3$ \\ $(\Delta / \sigma)_{\max }=0.002$ \\ $\Delta \rho_{\max }=1.75 \mathrm{e} \AA^{-3}$ \\ $\Delta \rho_{\min }=-0.93$ e $\AA^{-3}$ \\ Special details \\ Geometry. All esds (except the esd in the dihedral angle between two 1.s. planes) are estimated using the full covariance \\ matrix. The cell esds are taken into account individually in the estimation of esds in distances, angles and torsion angles; \\ correlations between esds in cell parameters are only used when they are defined by crystal symmetry. An approximate \\ (isotropic) treatment of cell esds is used for estimating esds involving l.s. planes.
}

Fractional atomic coordinates and isotropic or equivalent isotropic displacement parameters $\left(\AA^{2}\right)$

\begin{tabular}{llllll}
\hline & $x$ & $y$ & $z$ & $U_{\text {iso }} * U_{\text {eq }}$ & Occ. $(<1)$ \\
\hline $\mathrm{Ni}$ & $0.71466(3)$ & $1.00913(3)$ & $0.03328(2)$ & $0.02033(13)$ & \\
$\mathrm{N} 1$ & $0.7942(2)$ & $1.03447(17)$ & $-0.11157(17)$ & $0.0212(5)$ & \\
$\mathrm{N} 2$ & $0.6207(2)$ & $0.91916(17)$ & $0.02538(17)$ & $0.0210(5)$ & \\
$\mathrm{N} 3$ & $0.6076(2)$ & $0.96688(17)$ & $0.17567(17)$ & $0.0224(5)$ & \\
$\mathrm{N} 4$ & $0.8317(2)$ & $1.07621(18)$ & $0.04797(16)$ & $0.0229(5)$ & \\
$\mathrm{C} 1$ & $0.9005(2)$ & $1.0819(2)$ & $-0.19073(19)$ & $0.0223(5)$ & \\
$\mathrm{C} 2$ & $0.9285(3)$ & $1.0467(2)$ & $-0.2735(2)$ & $0.0247(6)$ & \\
$\mathrm{H} 2$ & 0.998662 & 1.066023 & -0.336441 & $0.030^{*}$ & \\
$\mathrm{C} 3$ & $0.8342(3)$ & $0.9777(2)$ & $-0.2468(2)$ & $0.0226(5)$ & \\
$\mathrm{C} 4$ & $0.7529(3)$ & $0.9711(2)$ & $-0.1464(2)$ & $0.0222(5)$ & \\
$\mathrm{C} 5$ & $0.9676(3)$ & $1.1629(2)$ & $-0.19148(19)$ & $0.0226(5)$ & \\
$\mathrm{C} 6$ & $0.9035(3)$ & $1.2478(2)$ & $-0.1678(2)$ & $0.0276(6)$ & $0.033^{*}$ \\
$\mathrm{H} 6$ & 0.813506 & 1.253780 & -0.150189 & $0.0333(7)$ & \\
$\mathrm{C} 7$ & $0.9693(3)$ & $1.3232(2)$ & $-0.1696(2)$ & $0.040^{*}$ & \\
$\mathrm{H} 7$ & 0.923784 & 1.379786 & -0.152441 & $0.0343(7)$ & \\
$\mathrm{C} 8$ & $1.1014(3)$ & $1.3173(2)$ & $-0.1963(2)$ & $0.0311(7)$ & \\
$\mathrm{C} 9$ & $1.1657(3)$ & $1.2342(2)$ & $-0.2225(2)$ & $0.037^{*}$ & \\
$\mathrm{H} 9$ & 1.255844 & 1.229402 & -0.242326 & $0.0266(6)$ & \\
$\mathrm{C} 10$ & $1.0997(3)$ & $1.1583(2)$ & $-0.2200(2)$ & \\
$\mathrm{H} 10$ & 1.145525 & 1.102123 & -0.238019 & $0.032^{*}$ &
\end{tabular}




\begin{tabular}{|c|c|c|c|c|}
\hline C11 & $1.1735(4)$ & $1.3984(3)$ & $-0.1962(3)$ & $0.0535(10)$ \\
\hline H11A & 1.217241 & 1.441655 & -0.268374 & $0.080^{*}$ \\
\hline H11B & 1.236159 & 1.365374 & -0.160591 & $0.080^{*}$ \\
\hline $\mathrm{H} 11 \mathrm{C}$ & 1.113791 & 1.440597 & -0.159493 & $0.080^{*}$ \\
\hline $\mathrm{C} 12$ & $0.8259(3)$ & $0.9241(2)$ & $-0.31327(19)$ & $0.0241(6)$ \\
\hline C13 & $0.9345(3)$ & $0.8812(2)$ & $-0.3679(2)$ & $0.0290(6)$ \\
\hline H13 & 1.014827 & 0.888036 & -0.364089 & $0.035^{*}$ \\
\hline C14 & $0.9268(3)$ & $0.8288(2)$ & $-0.4276(2)$ & $0.0332(7)$ \\
\hline H14 & 1.002180 & 0.800355 & -0.464251 & $0.040 *$ \\
\hline C15 & $0.8110(3)$ & $0.8167(2)$ & $-0.4353(2)$ & $0.0298(6)$ \\
\hline $\mathrm{C} 16$ & $0.7029(3)$ & $0.8629(2)$ & $-0.3836(2)$ & $0.0296(6)$ \\
\hline H16 & 0.622987 & 0.857461 & -0.389079 & $0.036^{*}$ \\
\hline $\mathrm{C} 17$ & $0.7102(3)$ & $0.9167(2)$ & $-0.3242(2)$ & $0.0273(6)$ \\
\hline H17 & 0.635718 & 0.948668 & -0.290877 & $0.033^{*}$ \\
\hline $\mathrm{C} 18$ & $0.8019(4)$ & $0.7559(3)$ & $-0.4967(3)$ & $0.0419(8)$ \\
\hline $\mathrm{H} 18 \mathrm{~A}$ & 0.783106 & 0.684718 & -0.450295 & $0.063 *$ \\
\hline H18B & 0.882819 & 0.758244 & -0.552870 & $0.063^{*}$ \\
\hline $\mathrm{H} 18 \mathrm{C}$ & 0.733704 & 0.785281 & -0.527028 & $0.063^{*}$ \\
\hline C19 & $0.6536(3)$ & $0.9030(2)$ & $-0.0665(2)$ & $0.0229(6)$ \\
\hline $\mathrm{C} 20$ & $0.5975(3)$ & $0.8240(2)$ & $-0.0690(2)$ & $0.0272(6)$ \\
\hline $\mathrm{H} 20$ & 0.618319 & 0.811856 & -0.132312 & $0.033^{*}$ \\
\hline $\mathrm{C} 21$ & $0.5111(3)$ & $0.7634(2)$ & $0.0217(2)$ & $0.0296(6)$ \\
\hline $\mathrm{H} 21$ & 0.471606 & 0.710148 & 0.019940 & $0.035^{*}$ \\
\hline $\mathrm{C} 22$ & $0.4807(3)$ & $0.7788(2)$ & $0.1153(2)$ & $0.0278(6)$ \\
\hline $\mathrm{H} 22$ & 0.421338 & 0.736637 & 0.177326 & $0.033^{*}$ \\
\hline $\mathrm{C} 23$ & $0.5394(2)$ & $0.8577(2)$ & $0.1162(2)$ & $0.0220(5)$ \\
\hline $\mathrm{C} 24$ & $0.5332(3)$ & $0.8847(2)$ & $0.2030(2)$ & $0.0222(5)$ \\
\hline $\mathrm{C} 25$ & $0.4838(3)$ & $0.8383(2)$ & $0.3115(2)$ & $0.0231(6)$ \\
\hline $\mathrm{C} 26$ & $0.5283(3)$ & $0.8943(2)$ & $0.3523(2)$ & $0.0244(6)$ \\
\hline H26 & 0.511937 & 0.880844 & 0.424332 & $0.029 *$ \\
\hline $\mathrm{C} 27$ & $0.6013(3)$ & $0.9739(2)$ & $0.2682(2)$ & $0.0223(5)$ \\
\hline $\mathrm{C} 28$ & $0.3986(3)$ & $0.7499(2)$ & $0.3716(2)$ & $0.0224(5)$ \\
\hline $\mathrm{C} 29$ & $0.2832(3)$ & $0.7518(2)$ & $0.3561(2)$ & $0.0275(6)$ \\
\hline H29 & 0.258054 & 0.811361 & 0.307600 & $0.033 *$ \\
\hline $\mathrm{C} 30$ & $0.2044(3)$ & $0.6683(2)$ & $0.4102(2)$ & $0.0280(6)$ \\
\hline $\mathrm{H} 30$ & 0.126830 & 0.671260 & 0.397359 & $0.034 *$ \\
\hline C31 & $0.2374(3)$ & $0.5802(2)$ & $0.4830(2)$ & $0.0290(6)$ \\
\hline C32 & $0.3523(3)$ & $0.5792(2)$ & $0.4990(2)$ & $0.0331(7)$ \\
\hline H32 & 0.376789 & 0.520100 & 0.548445 & $0.040 *$ \\
\hline $\mathrm{C} 33$ & $0.4316(3)$ & $0.6622(2)$ & $0.4446(2)$ & $0.0290(6)$ \\
\hline $\mathrm{H} 33$ & 0.509344 & 0.659241 & 0.457219 & $0.035^{*}$ \\
\hline C34 & $0.1504(3)$ & $0.4896(3)$ & $0.5423(3)$ & $0.0412(8)$ \\
\hline $\mathrm{H} 34 \mathrm{~A}$ & 0.198295 & 0.428387 & 0.572026 & $0.062 *$ \\
\hline H34B & 0.116748 & 0.478387 & 0.494491 & $0.062 *$ \\
\hline $\mathrm{H} 34 \mathrm{C}$ & 0.079749 & 0.503573 & 0.598715 & $0.062 *$ \\
\hline C35 & $0.6484(3)$ & $1.0604(2)$ & $0.2766(2)$ & $0.0263(6)$ \\
\hline $\mathrm{C} 36$ & $0.6888(3)$ & $1.0435(3)$ & $0.3615(2)$ & $0.0336(7)$ \\
\hline H36 & 0.689473 & 0.976019 & 0.410764 & $0.040^{*}$ \\
\hline
\end{tabular}




\begin{tabular}{|c|c|c|c|c|c|}
\hline C37 & $0.7279(3)$ & $1.1237(3)$ & $0.3746(3)$ & $0.0408(8)$ & \\
\hline H37 & 0.752837 & 1.110883 & 0.433680 & $0.049^{*}$ & \\
\hline C38 & $0.7310(3)$ & $1.2221(3)$ & $0.3028(3)$ & $0.0434(9)$ & \\
\hline C39 & $0.6875(3)$ & $1.2401(3)$ & 0.2198 (3) & $0.0410(8)$ & \\
\hline H39 & 0.686475 & 1.307769 & 0.171157 & $0.049^{*}$ & \\
\hline $\mathrm{C} 40$ & 0.6455 (3) & $1.1603(2)$ & $0.2071(2)$ & $0.0306(6)$ & \\
\hline $\mathrm{H} 40$ & 0.614683 & 1.174097 & 0.150804 & $0.037^{*}$ & \\
\hline $\mathrm{C} 41$ & $0.7823(4)$ & $1.3079(4)$ & $0.3137(4)$ & $0.0701(14)$ & \\
\hline $\mathrm{H} 41 \mathrm{~A}$ & 0.772811 & 1.291198 & 0.387242 & $0.105^{*}$ & \\
\hline H41B & 0.734805 & 1.371589 & 0.289428 & $0.105^{*}$ & \\
\hline $\mathrm{H} 41 \mathrm{C}$ & 0.872489 & 1.316474 & 0.271541 & $0.105^{*}$ & \\
\hline C42 & 0.9054 (3) & $1.1128(2)$ & $0.0596(2)$ & $0.0229(6)$ & \\
\hline $\mathrm{C} 43$ & $0.9938(3)$ & $1.1640(2)$ & $0.0752(2)$ & $0.0301(6)$ & \\
\hline $\mathrm{H} 43 \mathrm{~A}$ & 0.962221 & 1.233843 & 0.069903 & $0.045^{*}$ & \\
\hline H43B & 1.077168 & 1.166375 & 0.021547 & $0.045^{*}$ & \\
\hline $\mathrm{H} 43 \mathrm{C}$ & 1.001893 & 1.126315 & 0.144527 & $0.045^{*}$ & \\
\hline $\mathrm{C} 1 \mathrm{~A}$ & $0.5344(10)$ & $0.5285(8)$ & $-0.2757(10)$ & $0.090(3)$ & $0.779(5)$ \\
\hline H1AA & 0.557765 & 0.492990 & -0.325469 & $0.136^{*}$ & $0.779(5)$ \\
\hline $\mathrm{H} 1 \mathrm{AB}$ & 0.524481 & 0.602094 & -0.312423 & $0.136^{*}$ & $0.779(5)$ \\
\hline $\mathrm{H} 1 \mathrm{AC}$ & 0.453946 & 0.502305 & -0.220821 & $0.136^{*}$ & $0.779(5)$ \\
\hline C1B & $0.6389(7)$ & $0.5100(5)$ & $-0.2266(5)$ & $0.0786(19)$ & $0.779(5)$ \\
\hline H1BA & 0.648783 & 0.435829 & -0.188773 & $0.094 *$ & $0.779(5)$ \\
\hline H1BB & 0.720963 & 0.534607 & -0.281865 & $0.094^{*}$ & $0.779(5)$ \\
\hline $\mathrm{C} 1 \mathrm{C}$ & $0.6032(7)$ & $0.5668(6)$ & $-0.1514(6)$ & $0.0899(19)$ & $0.779(5)$ \\
\hline $\mathrm{H} 1 \mathrm{CA}$ & 0.594961 & 0.640780 & -0.190716 & $0.108^{*}$ & $0.779(5)$ \\
\hline $\mathrm{H} 1 \mathrm{CB}$ & 0.518935 & 0.543845 & -0.098965 & $0.108^{*}$ & $0.779(5)$ \\
\hline C1D & $0.6971(6)$ & $0.5521(5)$ & $-0.0944(5)$ & $0.0818(18)$ & $0.779(5)$ \\
\hline H1DA & 0.715769 & 0.477924 & -0.064400 & $0.098^{*}$ & $0.779(5)$ \\
\hline H1DB & 0.656402 & 0.576885 & -0.035486 & $0.098^{*}$ & $0.779(5)$ \\
\hline $\mathrm{C} 1 \mathrm{E}$ & $0.8225(6)$ & $0.6066(5)$ & $-0.1616(5)$ & $0.0698(17)$ & $0.779(5)$ \\
\hline H1EA & 0.806894 & 0.679286 & -0.200932 & $0.084^{*}$ & $0.779(5)$ \\
\hline H1EB & 0.874385 & 0.573326 & -0.211928 & $0.084^{*}$ & $0.779(5)$ \\
\hline $\mathrm{C} 1 \mathrm{~F}$ & 0.8895 (11) & $0.5976(8)$ & $-0.0849(8)$ & 0.114 & $0.779(5)$ \\
\hline H1FA & 0.976978 & 0.620915 & -0.123713 & $0.170 *$ & $0.779(5)$ \\
\hline H1FB & 0.890856 & 0.526034 & -0.038434 & $0.170^{*}$ & $0.779(5)$ \\
\hline H1FC & 0.843881 & 0.640080 & -0.043258 & $0.170^{*}$ & $0.779(5)$ \\
\hline $\mathrm{C} 1 \mathrm{G}$ & $0.519(4)$ & $0.501(2)$ & $-0.269(3)$ & $0.063(5)$ & $0.221(5)$ \\
\hline H1GA & 0.508682 & 0.549644 & -0.335107 & $0.095^{*}$ & $0.221(5)$ \\
\hline H1GB & 0.438516 & 0.466834 & -0.223634 & $0.095^{*}$ & $0.221(5)$ \\
\hline $\mathrm{H} 1 \mathrm{GC}$ & 0.585205 & 0.449655 & -0.284042 & $0.095^{*}$ & $0.221(5)$ \\
\hline $\mathrm{C} 1 \mathrm{H}$ & $0.5578(19)$ & $0.558(2)$ & $-0.215(2)$ & $0.084(4)$ & $0.221(5)$ \\
\hline H1HA & 0.504530 & 0.534871 & -0.140764 & $0.100 *$ & $0.221(5)$ \\
\hline H1HB & 0.539431 & 0.632029 & -0.246983 & $0.100 *$ & $0.221(5)$ \\
\hline C1I & $0.6978(19)$ & $0.543(2)$ & $-0.222(2)$ & 0.085 (4) & $0.221(5)$ \\
\hline H1IA & 0.715850 & 0.469931 & -0.187097 & $0.102 *$ & $0.221(5)$ \\
\hline H1IB & 0.751851 & 0.563699 & -0.296523 & $0.102 *$ & $0.221(5)$ \\
\hline $\mathrm{C} 1 \mathrm{~J}$ & $0.732(2)$ & $0.6058(17)$ & $-0.172(2)$ & $0.090(4)$ & $0.221(5)$ \\
\hline H1JA & 0.651340 & 0.636442 & -0.137639 & $0.108^{*}$ & $0.221(5)$ \\
\hline
\end{tabular}




\begin{tabular}{llllll} 
H1JB & 0.783165 & 0.663231 & -0.229621 & $0.108^{*}$ & $0.221(5)$ \\
C1K & $0.802(3)$ & $0.5613(16)$ & $-0.092(2)$ & $0.094(4)$ & $0.221(5)$ \\
H1KA & 0.856979 & 0.501915 & -0.103297 & $0.113^{*}$ & $0.221(5)$ \\
H1KB & 0.741166 & 0.538388 & -0.020175 & $0.113^{*}$ & $0.221(5)$ \\
C1L & $0.884(4)$ & $0.647(2)$ & $-0.108(3)$ & $0.078(5)$ & $0.221(5)$ \\
H1LA & 0.941234 & 0.670951 & -0.180777 & $0.116^{*}$ & $0.221(5)$ \\
H1LB & 0.934141 & 0.620361 & -0.060830 & $0.116^{*}$ & $0.221(5)$ \\
H1LC & 0.828534 & 0.703624 & -0.093759 & $0.116^{*}$ & $0.221(5)$ \\
\hline
\end{tabular}

Atomic displacement parameters $\left(\AA^{2}\right)$

\begin{tabular}{|c|c|c|c|c|c|c|}
\hline & $U^{11}$ & $U^{22}$ & $U^{33}$ & $U^{12}$ & $U^{13}$ & $U^{23}$ \\
\hline $\mathrm{Ni}$ & $0.0217(2)$ & $0.0251(2)$ & $0.01235(18)$ & $-0.00525(13)$ & $-0.00082(13)$ & $-0.00728(14)$ \\
\hline N1 & $0.0227(11)$ & $0.0236(11)$ & $0.0153(10)$ & $-0.0031(9)$ & $-0.0035(9)$ & $-0.0066(9)$ \\
\hline N2 & $0.0209(11)$ & $0.0276(12)$ & $0.0133(10)$ & $-0.0047(9)$ & $-0.0018(8)$ & $-0.0079(9)$ \\
\hline N3 & $0.0225(11)$ & $0.0271(12)$ & $0.0163(10)$ & $-0.0034(9)$ & $-0.0016(9)$ & $-0.0095(9)$ \\
\hline N4 & $0.0243(11)$ & $0.0282(12)$ & $0.0111(10)$ & $-0.0064(9)$ & $0.0010(8)$ & $-0.0058(9)$ \\
\hline $\mathrm{C} 1$ & $0.0210(13)$ & $0.0272(14)$ & $0.0125(11)$ & $-0.0021(11)$ & $-0.0009(10)$ & $-0.0043(10)$ \\
\hline $\mathrm{C} 2$ & $0.0250(13)$ & $0.0289(14)$ & $0.0151(12)$ & $-0.0008(11)$ & $-0.0017(10)$ & $-0.0070(11)$ \\
\hline $\mathrm{C} 3$ & $0.0247(13)$ & $0.0273(14)$ & $0.0135(12)$ & $0.0010(11)$ & $-0.0042(10)$ & $-0.0075(10)$ \\
\hline $\mathrm{C} 4$ & $0.0250(13)$ & $0.0261(14)$ & $0.0148(12)$ & $-0.0011(11)$ & $-0.0054(10)$ & $-0.0075(10)$ \\
\hline $\mathrm{C} 5$ & $0.0245(13)$ & $0.0260(13)$ & $0.0115(11)$ & $-0.0038(11)$ & $-0.0018(10)$ & $-0.0030(10)$ \\
\hline C6 & $0.0301(15)$ & $0.0299(15)$ & $0.0190(13)$ & $-0.0029(12)$ & $-0.0058(11)$ & $-0.0060(11)$ \\
\hline $\mathrm{C} 7$ & $0.0455(18)$ & $0.0264(15)$ & $0.0245(15)$ & $-0.0033(13)$ & $-0.0066(13)$ & $-0.0090(12)$ \\
\hline $\mathrm{C} 8$ & $0.0450(18)$ & $0.0346(16)$ & $0.0188(14)$ & $-0.0156(14)$ & $-0.0057(13)$ & $-0.0036(12)$ \\
\hline $\mathrm{C} 9$ & $0.0283(15)$ & $0.0400(17)$ & $0.0195(13)$ & $-0.0083(13)$ & $-0.0047(11)$ & $-0.0050(12)$ \\
\hline $\mathrm{C} 10$ & $0.0281(14)$ & $0.0294(15)$ & $0.0156(12)$ & $-0.0033(11)$ & $-0.0024(11)$ & $-0.0047(11)$ \\
\hline C11 & $0.065(3)$ & $0.053(2)$ & $0.045(2)$ & $-0.028(2)$ & $-0.0103(19)$ & $-0.0168(18)$ \\
\hline $\mathrm{C} 12$ & $0.0298(14)$ & $0.0270(14)$ & $0.0106(11)$ & $-0.0032(11)$ & $-0.0002(10)$ & $-0.0066(10)$ \\
\hline $\mathrm{C} 13$ & $0.0274(14)$ & $0.0364(16)$ & $0.0198(13)$ & $-0.0018(12)$ & $-0.0016(11)$ & $-0.0119(12)$ \\
\hline C14 & $0.0363(17)$ & $0.0378(17)$ & $0.0204(14)$ & $0.0005(13)$ & $0.0010(12)$ & $-0.0153(13)$ \\
\hline $\mathrm{C} 15$ & $0.0395(17)$ & $0.0325(15)$ & $0.0142(12)$ & $-0.0067(13)$ & $-0.0008(11)$ & $-0.0100(11)$ \\
\hline $\mathrm{C} 16$ & $0.0325(15)$ & $0.0398(16)$ & $0.0179(13)$ & $-0.0049(13)$ & $-0.0063(11)$ & $-0.0120(12)$ \\
\hline $\mathrm{C} 17$ & $0.0304(15)$ & $0.0353(15)$ & $0.0142(12)$ & $0.0002(12)$ & $-0.0030(11)$ & $-0.0111(11)$ \\
\hline $\mathrm{C} 18$ & $0.055(2)$ & $0.0460(19)$ & $0.0255(16)$ & $-0.0127(16)$ & $-0.0008(15)$ & $-0.0210(15)$ \\
\hline C19 & $0.0243(13)$ & $0.0290(14)$ & $0.0132(12)$ & $0.0002(11)$ & $-0.0030(10)$ & $-0.0085(10)$ \\
\hline $\mathrm{C} 20$ & $0.0299(15)$ & $0.0353(15)$ & $0.0169(13)$ & $-0.0067(12)$ & $-0.0039(11)$ & $-0.0110(12)$ \\
\hline $\mathrm{C} 21$ & $0.0316(15)$ & $0.0347(16)$ & $0.0232(14)$ & $-0.0116(12)$ & $-0.0042(12)$ & $-0.0114(12)$ \\
\hline $\mathrm{C} 22$ & $0.0270(14)$ & $0.0328(15)$ & $0.0189(13)$ & $-0.0110(12)$ & $0.0000(11)$ & $-0.0073(12)$ \\
\hline $\mathrm{C} 23$ & $0.0208(13)$ & $0.0268(13)$ & $0.0137(12)$ & $-0.0027(10)$ & $-0.0004(10)$ & $-0.0065(10)$ \\
\hline $\mathrm{C} 24$ & $0.0217(13)$ & $0.0260(13)$ & $0.0165(12)$ & $-0.0038(10)$ & $-0.0019(10)$ & $-0.0079(10)$ \\
\hline $\mathrm{C} 25$ & $0.0204(13)$ & $0.0289(14)$ & $0.0165(12)$ & $-0.0021(11)$ & $-0.0014(10)$ & $-0.0081(11)$ \\
\hline $\mathrm{C} 26$ & $0.0238(13)$ & $0.0330(15)$ & $0.0148(12)$ & $-0.0027(11)$ & $-0.0016(10)$ & $-0.0105(11)$ \\
\hline $\mathrm{C} 27$ & $0.0222(13)$ & $0.0282(14)$ & $0.0146(12)$ & $-0.0020(11)$ & $-0.0024(10)$ & $-0.0085(10)$ \\
\hline $\mathrm{C} 28$ & $0.0241(13)$ & $0.0243(13)$ & $0.0144(12)$ & $-0.0039(11)$ & $0.0001(10)$ & $-0.0069(10)$ \\
\hline C29 & $0.0290(14)$ & $0.0274(14)$ & $0.0186(13)$ & $-0.0016(11)$ & $-0.0043(11)$ & $-0.0033(11)$ \\
\hline $\mathrm{C} 30$ & $0.0250(14)$ & $0.0346(15)$ & $0.0223(14)$ & $-0.0048(12)$ & $-0.0035(11)$ & $-0.0104(12)$ \\
\hline C31 & $0.0340(16)$ & $0.0301(15)$ & $0.0202(13)$ & $-0.0075(12)$ & $-0.0019(12)$ & $-0.0101(12)$ \\
\hline
\end{tabular}




$\begin{array}{lllllll}\text { C32 } & 0.0402(17) & 0.0275(15) & 0.0252(15) & -0.0031(13) & -0.0100(13) & -0.0024(12) \\ \text { C33 } & 0.0275(14) & 0.0333(15) & 0.0241(14) & -0.0036(12) & -0.0077(12) & -0.0074(12) \\ \text { C34 } & 0.045(2) & 0.0354(17) & 0.0371(18) & -0.0169(15) & -0.0078(15) & -0.0053(14) \\ \text { C35 } & 0.0221(13) & 0.0374(16) & 0.0189(13) & -0.0037(12) & 0.0020(10) & -0.0164(12) \\ \text { C36 } & 0.0269(15) & 0.054(2) & 0.0222(14) & -0.0069(14) & 0.0008(11) & -0.0220(14) \\ \text { C37 } & 0.0291(16) & 0.066(2) & 0.0367(18) & -0.0086(15) & -0.0001(13) & -0.0357(18) \\ \text { C38 } & 0.0357(18) & 0.055(2) & 0.047(2) & -0.0112(15) & 0.0054(15) & -0.0389(18) \\ \text { C39 } & 0.0355(17) & 0.0375(18) & 0.046(2) & -0.0045(14) & 0.0027(15) & -0.0235(16) \\ \text { C40 } & 0.0254(14) & 0.0332(16) & 0.0290(15) & -0.0041(12) & 0.0008(12) & -0.0145(13) \\ \text { C41 } & 0.064(3) & 0.081(3) & 0.084(3) & -0.019(2) & 0.000(2) & -0.064(3) \\ \text { C42 } & 0.0268(14) & 0.0238(13) & 0.0143(12) & -0.0032(11) & -0.0034(10) & -0.0049(10) \\ \text { C43 } & 0.0299(15) & 0.0332(16) & 0.0292(15) & -0.0073(12) & -0.0112(12) & -0.0095(13) \\ \text { C1A } & 0.094(6) & 0.093(6) & 0.098(5) & -0.016(5) & -0.010(5) & -0.061(5) \\ \text { C1B } & 0.092(4) & 0.070(4) & 0.068(3) & -0.007(3) & -0.014(3) & -0.027(3) \\ \text { C1C } & 0.104(4) & 0.080(4) & 0.082(4) & -0.008(3) & -0.023(3) & -0.030(3) \\ \text { C1D } & 0.087(4) & 0.070(3) & 0.082(4) & -0.006(3) & -0.020(3) & -0.027(3) \\ \text { C1E } & 0.078(4) & 0.059(3) & 0.063(3) & -0.012(3) & -0.010(3) & -0.020(3) \\ \text { C1F } & 0.121(6) & 0.121(7) & 0.107(7) & -0.036(7) & -0.012(6) & -0.060(6) \\ \text { C1G } & 0.084(10) & 0.062(10) & 0.076(9) & -0.002(9) & -0.048(8) & -0.038(8) \\ \text { C1H } & 0.098(6) & 0.085(6) & 0.076(6) & -0.003(6) & -0.026(6) & -0.038(5) \\ \text { C1I } & 0.095(5) & 0.080(5) & 0.077(5) & -0.008(5) & -0.023(5) & -0.028(5) \\ \text { C1J } & 0.094(5) & 0.081(5) & 0.079(5) & -0.006(5) & -0.017(5) & -0.020(5) \\ \text { C1K } & 0.097(6) & 0.084(6) & 0.087(6) & -0.008(6) & -0.017(6) & -0.025(6) \\ \text { C1L } & 0.097(10) & 0.072(11) & 0.078(10) & -0.033(10) & -0.028(9) & -0.031(9) \\ & & & & & & \end{array}$

Geometric parameters $\left(\AA,{ }^{\circ}\right)$

\begin{tabular}{llll}
\hline $\mathrm{Ni}-\mathrm{N} 1$ & $1.896(2)$ & $\mathrm{C} 30-\mathrm{C} 31$ & $1.394(4)$ \\
$\mathrm{Ni}-\mathrm{N} 2$ & $1.846(2)$ & $\mathrm{C} 31-\mathrm{C} 32$ & $1.394(4)$ \\
$\mathrm{Ni}-\mathrm{N} 3$ & $1.906(2)$ & $\mathrm{C} 31-\mathrm{C} 34$ & $1.515(4)$ \\
$\mathrm{Ni}-\mathrm{N} 4$ & $1.861(2)$ & $\mathrm{C} 32-\mathrm{H} 32$ & 0.9500 \\
$\mathrm{~N} 1-\mathrm{C} 1$ & $1.375(3)$ & $\mathrm{C} 32-\mathrm{C} 33$ & $1.386(4)$ \\
$\mathrm{N} 1-\mathrm{C} 4$ & $1.397(3)$ & $\mathrm{C} 33-\mathrm{H} 33$ & 0.9500 \\
$\mathrm{~N} 2-\mathrm{C} 19$ & $1.365(3)$ & $\mathrm{C} 34-\mathrm{H} 34 \mathrm{~A}$ & 0.9800 \\
$\mathrm{~N} 2-\mathrm{C} 23$ & $1.362(3)$ & $\mathrm{C} 34-\mathrm{H} 34 \mathrm{~B}$ & 0.9800 \\
$\mathrm{~N} 3-\mathrm{C} 24$ & $1.390(3)$ & $\mathrm{C} 34-\mathrm{H} 34 \mathrm{C}$ & 0.9800 \\
$\mathrm{~N} 3-\mathrm{C} 27$ & $1.376(3)$ & $\mathrm{C} 35-\mathrm{C} 36$ & $1.401(4)$ \\
$\mathrm{N} 4-\mathrm{C} 42$ & $1.140(3)$ & $\mathrm{C} 35-\mathrm{C} 40$ & $1.391(4)$ \\
$\mathrm{C} 1-\mathrm{C} 2$ & $1.404(4)$ & $\mathrm{C} 36-\mathrm{H} 36$ & 0.9500 \\
$\mathrm{C} 1-\mathrm{C} 5$ & $1.469(4)$ & $\mathrm{C} 36-\mathrm{C} 37$ & $1.385(4)$ \\
$\mathrm{C} 2-\mathrm{H} 2$ & 0.9500 & $\mathrm{C} 37-\mathrm{H} 37$ & 0.9500 \\
$\mathrm{C} 2-\mathrm{C} 3$ & $1.407(4)$ & $\mathrm{C} 37-\mathrm{C} 38$ & $1.381(5)$ \\
$\mathrm{C} 3-\mathrm{C} 4$ & $1.403(4)$ & $\mathrm{C} 38-\mathrm{C} 39$ & $1.393(5)$ \\
$\mathrm{C} 3-\mathrm{C} 12$ & $1.485(4)$ & $\mathrm{C} 38-\mathrm{C} 41$ & $1.512(5)$ \\
$\mathrm{C} 4-\mathrm{C} 19$ & $1.445(4)$ & $\mathrm{C} 39-\mathrm{H} 39$ & 0.9500 \\
$\mathrm{C} 5-\mathrm{C} 6$ & $1.398(4)$ & $\mathrm{C} 39-\mathrm{C} 40$ & $1.393(4)$ \\
$\mathrm{C} 5-\mathrm{C} 10$ & $1.392(4)$ & $\mathrm{C} 40-\mathrm{H} 40$ & 0.9500 \\
$\mathrm{C} 6-\mathrm{H} 6$ & 0.9500 & $\mathrm{C} 41-\mathrm{H} 41 \mathrm{~A}$ & 0.9800
\end{tabular}




\begin{tabular}{|c|c|c|c|}
\hline $\mathrm{C} 6-\mathrm{C} 7$ & $1.384(4)$ & C41-H41B & 0.9800 \\
\hline $\mathrm{C} 7-\mathrm{H} 7$ & 0.9500 & $\mathrm{C} 41-\mathrm{H} 41 \mathrm{C}$ & 0.9800 \\
\hline $\mathrm{C} 7-\mathrm{C} 8$ & $1.394(5)$ & $\mathrm{C} 42-\mathrm{C} 43$ & $1.449(4)$ \\
\hline $\mathrm{C} 8-\mathrm{C} 9$ & $1.391(5)$ & $\mathrm{C} 43-\mathrm{H} 43 \mathrm{~A}$ & 0.9800 \\
\hline $\mathrm{C} 8-\mathrm{C} 11$ & $1.512(4)$ & $\mathrm{C} 43-\mathrm{H} 43 \mathrm{~B}$ & 0.9800 \\
\hline C9- $\mathrm{H} 9$ & 0.9500 & $\mathrm{C} 43-\mathrm{H} 43 \mathrm{C}$ & 0.9800 \\
\hline $\mathrm{C} 9-\mathrm{C} 10$ & $1.386(4)$ & $\mathrm{C} 1 \mathrm{~A}-\mathrm{H} 1 \mathrm{AA}$ & 0.9800 \\
\hline $\mathrm{C} 10-\mathrm{H} 10$ & 0.9500 & $\mathrm{C} 1 \mathrm{~A}-\mathrm{H} 1 \mathrm{AB}$ & 0.9800 \\
\hline C11-H11A & 0.9800 & $\mathrm{C} 1 \mathrm{~A}-\mathrm{H} 1 \mathrm{AC}$ & 0.9800 \\
\hline C11-H11B & 0.9800 & $\mathrm{C} 1 \mathrm{~A}-\mathrm{C} 1 \mathrm{~B}$ & $1.532(6)$ \\
\hline $\mathrm{C} 11-\mathrm{H} 11 \mathrm{C}$ & 0.9800 & $\mathrm{C} 1 \mathrm{~B}-\mathrm{H} 1 \mathrm{BA}$ & 0.9900 \\
\hline $\mathrm{C} 12-\mathrm{C} 13$ & $1.394(4)$ & $\mathrm{C} 1 \mathrm{~B}-\mathrm{H} 1 \mathrm{BB}$ & 0.9900 \\
\hline $\mathrm{C} 12-\mathrm{C} 17$ & $1.395(4)$ & $\mathrm{C} 1 \mathrm{~B}-\mathrm{C} 1 \mathrm{C}$ & $1.515(5)$ \\
\hline C13-H13 & 0.9500 & $\mathrm{C} 1 \mathrm{C}-\mathrm{H} 1 \mathrm{CA}$ & 0.9900 \\
\hline $\mathrm{C} 13-\mathrm{C} 14$ & $1.382(4)$ & $\mathrm{C} 1 \mathrm{C}-\mathrm{H} 1 \mathrm{CB}$ & 0.9900 \\
\hline C14-H14 & 0.9500 & $\mathrm{C} 1 \mathrm{C}-\mathrm{C} 1 \mathrm{D}$ & $1.515(5)$ \\
\hline $\mathrm{C} 14-\mathrm{C} 15$ & $1.393(4)$ & C1D-H1DA & 0.9900 \\
\hline $\mathrm{C} 15-\mathrm{C} 16$ & $1.397(4)$ & $\mathrm{C} 1 \mathrm{D}-\mathrm{H} 1 \mathrm{DB}$ & 0.9900 \\
\hline $\mathrm{C} 15-\mathrm{C} 18$ & $1.506(4)$ & $\mathrm{C} 1 \mathrm{D}-\mathrm{C} 1 \mathrm{E}$ & $1.525(5)$ \\
\hline $\mathrm{C} 16-\mathrm{H} 16$ & 0.9500 & $\mathrm{C} 1 \mathrm{E}-\mathrm{H} 1 \mathrm{EA}$ & 0.9900 \\
\hline $\mathrm{C} 16-\mathrm{C} 17$ & $1.393(4)$ & $\mathrm{C} 1 \mathrm{E}-\mathrm{H} 1 \mathrm{~EB}$ & 0.9900 \\
\hline C17-H17 & 0.9500 & $\mathrm{C} 1 \mathrm{E}-\mathrm{C} 1 \mathrm{~F}$ & $1.526(5)$ \\
\hline C18-H18A & 0.9800 & $\mathrm{C} 1 \mathrm{~F}-\mathrm{H} 1 \mathrm{FA}$ & 0.9800 \\
\hline $\mathrm{C} 18-\mathrm{H} 18 \mathrm{~B}$ & 0.9800 & $\mathrm{C} 1 \mathrm{~F}-\mathrm{H} 1 \mathrm{FB}$ & 0.9800 \\
\hline $\mathrm{C} 18-\mathrm{H} 18 \mathrm{C}$ & 0.9800 & $\mathrm{C} 1 \mathrm{~F}-\mathrm{H} 1 \mathrm{FC}$ & 0.9800 \\
\hline $\mathrm{C} 19-\mathrm{C} 20$ & $1.391(4)$ & $\mathrm{C} 1 \mathrm{G}-\mathrm{H} 1 \mathrm{GA}$ & 0.9800 \\
\hline $\mathrm{C} 20-\mathrm{H} 20$ & 0.9500 & $\mathrm{C} 1 \mathrm{G}-\mathrm{H} 1 \mathrm{~GB}$ & 0.9800 \\
\hline $\mathrm{C} 20-\mathrm{C} 21$ & $1.382(4)$ & $\mathrm{C} 1 \mathrm{G}-\mathrm{H} 1 \mathrm{GC}$ & 0.9800 \\
\hline $\mathrm{C} 21-\mathrm{H} 21$ & 0.9500 & $\mathrm{C} 1 \mathrm{G}-\mathrm{C} 1 \mathrm{H}$ & $1.529(6)$ \\
\hline $\mathrm{C} 21-\mathrm{C} 22$ & $1.387(4)$ & $\mathrm{C} 1 \mathrm{H}-\mathrm{H} 1 \mathrm{HA}$ & 0.9900 \\
\hline $\mathrm{C} 22-\mathrm{H} 22$ & 0.9500 & $\mathrm{C} 1 \mathrm{H}-\mathrm{H} 1 \mathrm{HB}$ & 0.9900 \\
\hline $\mathrm{C} 22-\mathrm{C} 23$ & $1.395(4)$ & $\mathrm{C} 1 \mathrm{H}-\mathrm{C} 1 \mathrm{I}$ & $1.530(6)$ \\
\hline $\mathrm{C} 23-\mathrm{C} 24$ & $1.443(4)$ & C1I-H1IA & 0.9900 \\
\hline $\mathrm{C} 24-\mathrm{C} 25$ & $1.396(4)$ & $\mathrm{C} 1 \mathrm{I}-\mathrm{H} 1 \mathrm{IB}$ & 0.9900 \\
\hline $\mathrm{C} 25-\mathrm{C} 26$ & $1.399(4)$ & $\mathrm{C} 1 \mathrm{I}-\mathrm{C} 1 \mathrm{~J}$ & $1.519(6)$ \\
\hline $\mathrm{C} 25-\mathrm{C} 28$ & $1.481(4)$ & $\mathrm{C} 1 \mathrm{~J}-\mathrm{H} 1 \mathrm{JA}$ & 0.9900 \\
\hline $\mathrm{C} 26-\mathrm{H} 26$ & 0.9500 & $\mathrm{C} 1 \mathrm{~J}-\mathrm{H} 1 \mathrm{JB}$ & 0.9900 \\
\hline $\mathrm{C} 26-\mathrm{C} 27$ & $1.401(4)$ & $\mathrm{C} 1 \mathrm{~J}-\mathrm{C} 1 \mathrm{~K}$ & $1.526(6)$ \\
\hline $\mathrm{C} 27-\mathrm{C} 35$ & $1.476(4)$ & $\mathrm{C} 1 \mathrm{~K}-\mathrm{H} 1 \mathrm{KA}$ & 0.9900 \\
\hline $\mathrm{C} 28-\mathrm{C} 29$ & $1.393(4)$ & $\mathrm{C} 1 \mathrm{~K}-\mathrm{H} 1 \mathrm{~KB}$ & 0.9900 \\
\hline $\mathrm{C} 28-\mathrm{C} 33$ & $1.392(4)$ & $\mathrm{C} 1 \mathrm{~K}-\mathrm{C} 1 \mathrm{~L}$ & $1.533(6)$ \\
\hline $\mathrm{C} 29-\mathrm{H} 29$ & 0.9500 & C1L-H1LA & 0.9800 \\
\hline $\mathrm{C} 29-\mathrm{C} 30$ & $1.387(4)$ & $\mathrm{C} 1 \mathrm{~L}-\mathrm{H} 1 \mathrm{LB}$ & 0.9800 \\
\hline $\mathrm{C} 30-\mathrm{H} 30$ & 0.9500 & $\mathrm{C} 1 \mathrm{~L}-\mathrm{H} 1 \mathrm{LC}$ & 0.9800 \\
\hline $\mathrm{N} 1-\mathrm{Ni}-\mathrm{N} 3$ & $166.68(9)$ & $\mathrm{C} 31-\mathrm{C} 32-\mathrm{H} 32$ & 119.2 \\
\hline $\mathrm{N} 2-\mathrm{Ni}-\mathrm{N} 1$ & $83.38(9)$ & $\mathrm{C} 33-\mathrm{C} 32-\mathrm{C} 31$ & $121.6(3)$ \\
\hline $\mathrm{N} 2-\mathrm{Ni}-\mathrm{N} 3$ & $83.52(9)$ & $\mathrm{C} 33-\mathrm{C} 32-\mathrm{H} 32$ & 119.2 \\
\hline
\end{tabular}




\begin{tabular}{|c|c|c|c|}
\hline $\mathrm{N} 2-\mathrm{Ni}-\mathrm{N} 4$ & $168.06(10)$ & $\mathrm{C} 28-\mathrm{C} 33-\mathrm{H} 33$ & 119.7 \\
\hline $\mathrm{N} 4-\mathrm{Ni}-\mathrm{N} 1$ & $96.77(9)$ & $\mathrm{C} 32-\mathrm{C} 33-\mathrm{C} 28$ & $120.6(3)$ \\
\hline $\mathrm{N} 4-\mathrm{Ni}-\mathrm{N} 3$ & $96.54(10)$ & $\mathrm{C} 32-\mathrm{C} 33-\mathrm{H} 33$ & 119.7 \\
\hline $\mathrm{C} 1-\mathrm{N} 1-\mathrm{Ni}$ & $138.26(19)$ & $\mathrm{C} 31-\mathrm{C} 34-\mathrm{H} 34 \mathrm{~A}$ & 109.5 \\
\hline $\mathrm{C} 1-\mathrm{N} 1-\mathrm{C} 4$ & $106.5(2)$ & C $31-\mathrm{C} 34-\mathrm{H} 34 \mathrm{~B}$ & 109.5 \\
\hline $\mathrm{C} 4-\mathrm{N} 1-\mathrm{Ni}$ & $113.43(17)$ & $\mathrm{C} 31-\mathrm{C} 34-\mathrm{H} 34 \mathrm{C}$ & 109.5 \\
\hline $\mathrm{C} 19-\mathrm{N} 2-\mathrm{Ni}$ & $118.35(18)$ & $\mathrm{H} 34 \mathrm{~A}-\mathrm{C} 34-\mathrm{H} 34 \mathrm{~B}$ & 109.5 \\
\hline $\mathrm{C} 23-\mathrm{N} 2-\mathrm{Ni}$ & $117.87(18)$ & $\mathrm{H} 34 \mathrm{~A}-\mathrm{C} 34-\mathrm{H} 34 \mathrm{C}$ & 109.5 \\
\hline $\mathrm{C} 23-\mathrm{N} 2-\mathrm{C} 19$ & $122.5(2)$ & $\mathrm{H} 34 \mathrm{~B}-\mathrm{C} 34-\mathrm{H} 34 \mathrm{C}$ & 109.5 \\
\hline $\mathrm{C} 24-\mathrm{N} 3-\mathrm{Ni}$ & $112.48(17)$ & $\mathrm{C} 36-\mathrm{C} 35-\mathrm{C} 27$ & $119.3(3)$ \\
\hline $\mathrm{C} 27-\mathrm{N} 3-\mathrm{Ni}$ & $138.85(19)$ & $\mathrm{C} 40-\mathrm{C} 35-\mathrm{C} 27$ & $122.2(3)$ \\
\hline $\mathrm{C} 27-\mathrm{N} 3-\mathrm{C} 24$ & $106.1(2)$ & $\mathrm{C} 40-\mathrm{C} 35-\mathrm{C} 36$ & $118.3(3)$ \\
\hline $\mathrm{C} 42-\mathrm{N} 4-\mathrm{Ni}$ & $176.7(2)$ & $\mathrm{C} 35-\mathrm{C} 36-\mathrm{H} 36$ & 119.6 \\
\hline $\mathrm{N} 1-\mathrm{C} 1-\mathrm{C} 2$ & $109.5(2)$ & $\mathrm{C} 37-\mathrm{C} 36-\mathrm{C} 35$ & $120.9(3)$ \\
\hline $\mathrm{N} 1-\mathrm{C} 1-\mathrm{C} 5$ & $124.8(2)$ & $\mathrm{C} 37-\mathrm{C} 36-\mathrm{H} 36$ & 119.6 \\
\hline $\mathrm{C} 2-\mathrm{C} 1-\mathrm{C} 5$ & $125.5(2)$ & $\mathrm{C} 36-\mathrm{C} 37-\mathrm{H} 37$ & 119.6 \\
\hline $\mathrm{C} 1-\mathrm{C} 2-\mathrm{H} 2$ & 126.0 & $\mathrm{C} 38-\mathrm{C} 37-\mathrm{C} 36$ & $120.8(3)$ \\
\hline $\mathrm{C} 1-\mathrm{C} 2-\mathrm{C} 3$ & $107.9(2)$ & $\mathrm{C} 38-\mathrm{C} 37-\mathrm{H} 37$ & 119.6 \\
\hline $\mathrm{C} 3-\mathrm{C} 2-\mathrm{H} 2$ & 126.0 & $\mathrm{C} 37-\mathrm{C} 38-\mathrm{C} 39$ & $118.6(3)$ \\
\hline $\mathrm{C} 2-\mathrm{C} 3-\mathrm{C} 12$ & $125.8(2)$ & $\mathrm{C} 37-\mathrm{C} 38-\mathrm{C} 41$ & $120.4(4)$ \\
\hline $\mathrm{C} 4-\mathrm{C} 3-\mathrm{C} 2$ & $105.8(2)$ & $\mathrm{C} 39-\mathrm{C} 38-\mathrm{C} 41$ & $120.9(4)$ \\
\hline $\mathrm{C} 4-\mathrm{C} 3-\mathrm{C} 12$ & $128.4(3)$ & $\mathrm{C} 38-\mathrm{C} 39-\mathrm{H} 39$ & 119.5 \\
\hline $\mathrm{N} 1-\mathrm{C} 4-\mathrm{C} 3$ & $110.2(2)$ & $\mathrm{C} 38-\mathrm{C} 39-\mathrm{C} 40$ & $121.0(3)$ \\
\hline $\mathrm{N} 1-\mathrm{C} 4-\mathrm{C} 19$ & $113.8(2)$ & $\mathrm{C} 40-\mathrm{C} 39-\mathrm{H} 39$ & 119.5 \\
\hline $\mathrm{C} 3-\mathrm{C} 4-\mathrm{C} 19$ & $134.9(3)$ & $\mathrm{C} 35-\mathrm{C} 40-\mathrm{C} 39$ & $120.3(3)$ \\
\hline $\mathrm{C} 6-\mathrm{C} 5-\mathrm{C} 1$ & $122.1(2)$ & $\mathrm{C} 35-\mathrm{C} 40-\mathrm{H} 40$ & 119.9 \\
\hline $\mathrm{C} 10-\mathrm{C} 5-\mathrm{C} 1$ & $119.9(3)$ & $\mathrm{C} 39-\mathrm{C} 40-\mathrm{H} 40$ & 119.9 \\
\hline $\mathrm{C} 10-\mathrm{C} 5-\mathrm{C} 6$ & $117.9(3)$ & $\mathrm{C} 38-\mathrm{C} 41-\mathrm{H} 41 \mathrm{~A}$ & 109.5 \\
\hline $\mathrm{C} 5-\mathrm{C} 6-\mathrm{H} 6$ & 119.6 & $\mathrm{C} 38-\mathrm{C} 41-\mathrm{H} 41 \mathrm{~B}$ & 109.5 \\
\hline $\mathrm{C} 7-\mathrm{C} 6-\mathrm{C} 5$ & $120.8(3)$ & $\mathrm{C} 38-\mathrm{C} 41-\mathrm{H} 41 \mathrm{C}$ & 109.5 \\
\hline $\mathrm{C} 7-\mathrm{C} 6-\mathrm{H} 6$ & 119.6 & $\mathrm{H} 41 \mathrm{~A}-\mathrm{C} 41-\mathrm{H} 41 \mathrm{~B}$ & 109.5 \\
\hline $\mathrm{C} 6-\mathrm{C} 7-\mathrm{H} 7$ & 119.5 & $\mathrm{H} 41 \mathrm{~A}-\mathrm{C} 41-\mathrm{H} 41 \mathrm{C}$ & 109.5 \\
\hline $\mathrm{C} 6-\mathrm{C} 7-\mathrm{C} 8$ & $121.1(3)$ & $\mathrm{H} 41 \mathrm{~B}-\mathrm{C} 41-\mathrm{H} 41 \mathrm{C}$ & 109.5 \\
\hline $\mathrm{C} 8-\mathrm{C} 7-\mathrm{H} 7$ & 119.5 & $\mathrm{~N} 4-\mathrm{C} 42-\mathrm{C} 43$ & $176.7(3)$ \\
\hline $\mathrm{C} 7-\mathrm{C} 8-\mathrm{C} 11$ & $121.2(3)$ & $\mathrm{C} 42-\mathrm{C} 43-\mathrm{H} 43 \mathrm{~A}$ & 109.5 \\
\hline $\mathrm{C} 9-\mathrm{C} 8-\mathrm{C} 7$ & $118.2(3)$ & $\mathrm{C} 42-\mathrm{C} 43-\mathrm{H} 43 \mathrm{~B}$ & 109.5 \\
\hline $\mathrm{C} 9-\mathrm{C} 8-\mathrm{C} 11$ & $120.6(3)$ & $\mathrm{C} 42-\mathrm{C} 43-\mathrm{H} 43 \mathrm{C}$ & 109.5 \\
\hline $\mathrm{C} 8-\mathrm{C} 9-\mathrm{H} 9$ & 119.6 & $\mathrm{H} 43 \mathrm{~A}-\mathrm{C} 43-\mathrm{H} 43 \mathrm{~B}$ & 109.5 \\
\hline $\mathrm{C} 10-\mathrm{C} 9-\mathrm{C} 8$ & $120.8(3)$ & $\mathrm{H} 43 \mathrm{~A}-\mathrm{C} 43-\mathrm{H} 43 \mathrm{C}$ & 109.5 \\
\hline $\mathrm{C} 10-\mathrm{C} 9-\mathrm{H} 9$ & 119.6 & $\mathrm{H} 43 \mathrm{~B}-\mathrm{C} 43-\mathrm{H} 43 \mathrm{C}$ & 109.5 \\
\hline $\mathrm{C} 5-\mathrm{C} 10-\mathrm{H} 10$ & 119.4 & $\mathrm{H} 1 \mathrm{AA}-\mathrm{C} 1 \mathrm{~A}-\mathrm{H} 1 \mathrm{AB}$ & 109.5 \\
\hline $\mathrm{C} 9-\mathrm{C} 10-\mathrm{C} 5$ & $121.2(3)$ & $\mathrm{H} 1 \mathrm{AA}-\mathrm{C} 1 \mathrm{~A}-\mathrm{H} 1 \mathrm{AC}$ & 109.5 \\
\hline $\mathrm{C} 9-\mathrm{C} 10-\mathrm{H} 10$ & 119.4 & $\mathrm{H} 1 \mathrm{AB}-\mathrm{C} 1 \mathrm{~A}-\mathrm{H} 1 \mathrm{AC}$ & 109.5 \\
\hline $\mathrm{C} 8-\mathrm{C} 11-\mathrm{H} 11 \mathrm{~A}$ & 109.5 & $\mathrm{C} 1 \mathrm{~B}-\mathrm{C} 1 \mathrm{~A}-\mathrm{H} 1 \mathrm{AA}$ & 109.5 \\
\hline $\mathrm{C} 8-\mathrm{C} 11-\mathrm{H} 11 \mathrm{~B}$ & 109.5 & $\mathrm{C} 1 \mathrm{~B}-\mathrm{C} 1 \mathrm{~A}-\mathrm{H} 1 \mathrm{AB}$ & 109.5 \\
\hline $\mathrm{C} 8-\mathrm{C} 11-\mathrm{H} 11 \mathrm{C}$ & 109.5 & $\mathrm{C} 1 \mathrm{~B}-\mathrm{C} 1 \mathrm{~A}-\mathrm{H} 1 \mathrm{AC}$ & 109.5 \\
\hline $\mathrm{H} 11 \mathrm{~A}-\mathrm{C} 11-\mathrm{H} 11 \mathrm{~B}$ & 109.5 & $\mathrm{C} 1 \mathrm{~A}-\mathrm{C} 1 \mathrm{~B}-\mathrm{H} 1 \mathrm{BA}$ & 109.9 \\
\hline $\mathrm{H} 11 \mathrm{~A}-\mathrm{C} 11-\mathrm{H} 11 \mathrm{C}$ & 109.5 & $\mathrm{C} 1 \mathrm{~A}-\mathrm{C} 1 \mathrm{~B}-\mathrm{H} 1 \mathrm{BB}$ & 109.9 \\
\hline
\end{tabular}




\begin{tabular}{|c|c|}
\hline $\mathrm{H} 11 \mathrm{~B}-\mathrm{C} 11-\mathrm{H} 11 \mathrm{C}$ & 109.5 \\
\hline $\mathrm{C} 13-\mathrm{C} 12-\mathrm{C} 3$ & $120.6(3)$ \\
\hline $\mathrm{C} 13-\mathrm{C} 12-\mathrm{C} 17$ & $118.2(3)$ \\
\hline $\mathrm{C} 17-\mathrm{C} 12-\mathrm{C} 3$ & $121.2(3)$ \\
\hline $\mathrm{C} 12-\mathrm{C} 13-\mathrm{H} 13$ & 119.6 \\
\hline $\mathrm{C} 14-\mathrm{C} 13-\mathrm{C} 12$ & $120.8(3)$ \\
\hline $\mathrm{C} 14-\mathrm{C} 13-\mathrm{H} 13$ & 119.6 \\
\hline $\mathrm{C} 13-\mathrm{C} 14-\mathrm{H} 14$ & 119.2 \\
\hline $\mathrm{C} 13-\mathrm{C} 14-\mathrm{C} 15$ & $121.5(3)$ \\
\hline $\mathrm{C} 15-\mathrm{C} 14-\mathrm{H} 14$ & 119.2 \\
\hline $\mathrm{C} 14-\mathrm{C} 15-\mathrm{C} 16$ & $117.6(3)$ \\
\hline $\mathrm{C} 14-\mathrm{C} 15-\mathrm{C} 18$ & $121.5(3)$ \\
\hline $\mathrm{C} 16-\mathrm{C} 15-\mathrm{C} 18$ & $120.9(3)$ \\
\hline $\mathrm{C} 15-\mathrm{C} 16-\mathrm{H} 16$ & 119.4 \\
\hline $\mathrm{C} 17-\mathrm{C} 16-\mathrm{C} 15$ & $121.1(3)$ \\
\hline $\mathrm{C} 17-\mathrm{C} 16-\mathrm{H} 16$ & 119.4 \\
\hline $\mathrm{C} 12-\mathrm{C} 17-\mathrm{H} 17$ & 119.7 \\
\hline $\mathrm{C} 16-\mathrm{C} 17-\mathrm{C} 12$ & $120.6(3)$ \\
\hline $\mathrm{C} 16-\mathrm{C} 17-\mathrm{H} 17$ & 119.7 \\
\hline $\mathrm{C} 15-\mathrm{C} 18-\mathrm{H} 18 \mathrm{~A}$ & 109.5 \\
\hline $\mathrm{C} 15-\mathrm{C} 18-\mathrm{H} 18 \mathrm{~B}$ & 109.5 \\
\hline $\mathrm{C} 15-\mathrm{C} 18-\mathrm{H} 18 \mathrm{C}$ & 109.5 \\
\hline $\mathrm{H} 18 \mathrm{~A}-\mathrm{C} 18-\mathrm{H} 18 \mathrm{~B}$ & 109.5 \\
\hline $\mathrm{H} 18 \mathrm{~A}-\mathrm{C} 18-\mathrm{H} 18 \mathrm{C}$ & 109.5 \\
\hline $\mathrm{H} 18 \mathrm{~B}-\mathrm{C} 18-\mathrm{H} 18 \mathrm{C}$ & 109.5 \\
\hline $\mathrm{N} 2-\mathrm{C} 19-\mathrm{C} 4$ & $110.6(2)$ \\
\hline $\mathrm{N} 2-\mathrm{C} 19-\mathrm{C} 20$ & $119.0(2)$ \\
\hline $\mathrm{C} 20-\mathrm{C} 19-\mathrm{C} 4$ & $130.2(2)$ \\
\hline $\mathrm{C} 19-\mathrm{C} 20-\mathrm{H} 20$ & 120.4 \\
\hline $\mathrm{C} 21-\mathrm{C} 20-\mathrm{C} 19$ & $119.1(3)$ \\
\hline $\mathrm{C} 21-\mathrm{C} 20-\mathrm{H} 20$ & 120.4 \\
\hline $\mathrm{C} 20-\mathrm{C} 21-\mathrm{H} 21$ & 119.3 \\
\hline $\mathrm{C} 20-\mathrm{C} 21-\mathrm{C} 22$ & $121.4(3)$ \\
\hline $\mathrm{C} 22-\mathrm{C} 21-\mathrm{H} 21$ & 119.3 \\
\hline $\mathrm{C} 21-\mathrm{C} 22-\mathrm{H} 22$ & 120.8 \\
\hline $\mathrm{C} 21-\mathrm{C} 22-\mathrm{C} 23$ & $118.4(2)$ \\
\hline $\mathrm{C} 23-\mathrm{C} 22-\mathrm{H} 22$ & 120.8 \\
\hline $\mathrm{N} 2-\mathrm{C} 23-\mathrm{C} 22$ & $119.5(2)$ \\
\hline $\mathrm{N} 2-\mathrm{C} 23-\mathrm{C} 24$ & $110.7(2)$ \\
\hline $\mathrm{C} 22-\mathrm{C} 23-\mathrm{C} 24$ & $129.7(2)$ \\
\hline $\mathrm{N} 3-\mathrm{C} 24-\mathrm{C} 23$ & $114.4(2)$ \\
\hline $\mathrm{N} 3-\mathrm{C} 24-\mathrm{C} 25$ & $110.6(2)$ \\
\hline $\mathrm{C} 25-\mathrm{C} 24-\mathrm{C} 23$ & $134.3(3)$ \\
\hline $\mathrm{C} 24-\mathrm{C} 25-\mathrm{C} 26$ & $105.9(2)$ \\
\hline $\mathrm{C} 24-\mathrm{C} 25-\mathrm{C} 28$ & $127.3(3)$ \\
\hline $\mathrm{C} 26-\mathrm{C} 25-\mathrm{C} 28$ & $126.8(2)$ \\
\hline $\mathrm{C} 25-\mathrm{C} 26-\mathrm{H} 26$ & 126.1 \\
\hline $\mathrm{C} 25-\mathrm{C} 26-\mathrm{C} 27$ & $107.9(2)$ \\
\hline
\end{tabular}

$\begin{array}{ll}\text { H1BA-C1B-H1BB } & 108.3 \\ \text { C1C-C1B-C1A } & 109.1(7) \\ \text { C1C-C1B-H1BA } & 109.9 \\ \text { C1C-C1B-H1BB } & 109.9 \\ \text { C1B-C1C-H1CA } & 108.6 \\ \text { C1B-C1C-H1CB } & 108.6 \\ \text { H1CA-C1C-H1CB } & 107.6 \\ \text { C1D-C1C-C1B } & 114.6(6) \\ \text { C1D-C1C-H1CA } & 108.6 \\ \text { C1D-C1C-H1CB } & 108.6 \\ \text { C1C-C1D-H1DA } & 108.5 \\ \text { C1C-C1D-H1DB } & 108.5 \\ \text { C1C-C1D-C1E } & 114.9(6)\end{array}$

H1DA-C1D-H1DB $\quad 107.5$

C1E-C1D-H1DA $\quad 108.5$

C1E-C1D-H1DB $\quad 108.5$

C1D-C1E-H1EA $\quad 110.7$

C1D-C1E-H1EB $\quad 110.7$

$\mathrm{C} 1 \mathrm{D}-\mathrm{C} 1 \mathrm{E}-\mathrm{C} 1 \mathrm{~F} \quad 105.3(6)$

H1EA-C1E-H1EB $\quad 108.8$

C1F-C1E-H1EA $\quad 110.7$

$\mathrm{C} 1 \mathrm{~F}-\mathrm{C} 1 \mathrm{E}-\mathrm{H} 1 \mathrm{~EB} \quad 110.7$

C1E-C1F-H1FA $\quad 109.5$

$\mathrm{C} 1 \mathrm{E}-\mathrm{C} 1 \mathrm{~F}-\mathrm{H} 1 \mathrm{FB} \quad 109.5$

$\mathrm{C} 1 \mathrm{E}-\mathrm{C} 1 \mathrm{~F}-\mathrm{H} 1 \mathrm{FC} \quad 109.5$

H1FA-C1F-H1FB $\quad 109.5$

$\mathrm{H} 1 \mathrm{FA}-\mathrm{C} 1 \mathrm{~F}-\mathrm{H} 1 \mathrm{FC} \quad 109.5$

H1FB - C1F-H1FC $\quad 109.5$

H1GA-C1G-H1GB $\quad 109.5$

$\mathrm{H} 1 \mathrm{GA}-\mathrm{C} 1 \mathrm{G}-\mathrm{H} 1 \mathrm{GC} \quad 109.5$

$\mathrm{H} 1 \mathrm{~GB}-\mathrm{C} 1 \mathrm{G}-\mathrm{H} 1 \mathrm{GC} \quad 109.5$

$\mathrm{C} 1 \mathrm{H}-\mathrm{C} 1 \mathrm{G}-\mathrm{H} 1 \mathrm{GA} \quad 109.5$

$\mathrm{C} 1 \mathrm{H}-\mathrm{C} 1 \mathrm{G}-\mathrm{H} 1 \mathrm{~GB} \quad 109.5$

$\mathrm{C} 1 \mathrm{H}-\mathrm{C} 1 \mathrm{G}-\mathrm{H} 1 \mathrm{GC} \quad 109.5$

$\mathrm{C} 1 \mathrm{G}-\mathrm{C} 1 \mathrm{H}-\mathrm{H} 1 \mathrm{HA} \quad 108.6$

$\mathrm{C} 1 \mathrm{G}-\mathrm{C} 1 \mathrm{H}-\mathrm{H} 1 \mathrm{HB} \quad 108.6$

$\mathrm{C} 1 \mathrm{G}-\mathrm{C} 1 \mathrm{H}-\mathrm{C} 1 \mathrm{I} \quad 114.6(17)$

$\mathrm{H} 1 \mathrm{HA}-\mathrm{C} 1 \mathrm{H}-\mathrm{H} 1 \mathrm{HB} \quad 107.6$

$\mathrm{C} 1 \mathrm{I}-\mathrm{C} 1 \mathrm{H}-\mathrm{H} 1 \mathrm{HA} \quad 108.6$

$\mathrm{C} 1 \mathrm{I}-\mathrm{C} 1 \mathrm{H}-\mathrm{H} 1 \mathrm{HB} \quad 108.6$

$\mathrm{C} 1 \mathrm{H}-\mathrm{C} 1 \mathrm{I}-\mathrm{H} 1 \mathrm{IA} \quad 109.2$

$\mathrm{C} 1 \mathrm{H}-\mathrm{C} 1 \mathrm{I}-\mathrm{H} 1 \mathrm{IB} \quad 109.2$

H1IA-C1I-H1IB $\quad 107.9$

$\mathrm{C} 1 \mathrm{~J}-\mathrm{C} 1 \mathrm{I}-\mathrm{C} 1 \mathrm{H} \quad 112.2(15)$

C1J-C1I-H1IA $\quad 109.2$

C1J-C1I-H1IB $\quad 109.2$

$\mathrm{C} 1 \mathrm{I}-\mathrm{C} 1 \mathrm{~J}-\mathrm{H} 1 \mathrm{JA} \quad 106.4$

$\mathrm{C} 1 \mathrm{I}-\mathrm{C} 1 \mathrm{~J}-\mathrm{H} 1 \mathrm{JB} \quad 106.4$ 


\begin{tabular}{|c|c|c|c|}
\hline $\mathrm{C} 27-\mathrm{C} 26-\mathrm{H} 26$ & 126.1 & $\mathrm{C} 1 \mathrm{I}-\mathrm{C} 1 \mathrm{~J}-\mathrm{C} 1 \mathrm{~K}$ & $123.6(18)$ \\
\hline $\mathrm{N} 3-\mathrm{C} 27-\mathrm{C} 26$ & $109.5(2)$ & $\mathrm{H} 1 \mathrm{JA}-\mathrm{C} 1 \mathrm{~J}-\mathrm{H} 1 \mathrm{JB}$ & 106.5 \\
\hline N3-C27-C35 & $124.7(2)$ & $\mathrm{C} 1 \mathrm{~K}-\mathrm{C} 1 \mathrm{~J}-\mathrm{H} 1 \mathrm{JA}$ & 106.4 \\
\hline $\mathrm{C} 26-\mathrm{C} 27-\mathrm{C} 35$ & $125.3(2)$ & $\mathrm{C} 1 \mathrm{~K}-\mathrm{C} 1 \mathrm{~J}-\mathrm{H} 1 \mathrm{JB}$ & 106.4 \\
\hline $\mathrm{C} 29-\mathrm{C} 28-\mathrm{C} 25$ & $120.9(2)$ & $\mathrm{C} 1 \mathrm{~J}-\mathrm{C} 1 \mathrm{~K}-\mathrm{H} 1 \mathrm{KA}$ & 110.4 \\
\hline $\mathrm{C} 33-\mathrm{C} 28-\mathrm{C} 25$ & $121.1(3)$ & $\mathrm{C} 1 \mathrm{~J}-\mathrm{C} 1 \mathrm{~K}-\mathrm{H} 1 \mathrm{~KB}$ & 110.4 \\
\hline $\mathrm{C} 33-\mathrm{C} 28-\mathrm{C} 29$ & $118.0(2)$ & $\mathrm{C} 1 \mathrm{~J}-\mathrm{C} 1 \mathrm{~K}-\mathrm{C} 1 \mathrm{~L}$ & $106.8(15)$ \\
\hline $\mathrm{C} 28-\mathrm{C} 29-\mathrm{H} 29$ & 119.4 & $\mathrm{H} 1 \mathrm{KA}-\mathrm{C} 1 \mathrm{~K}-\mathrm{H} 1 \mathrm{~KB}$ & 108.6 \\
\hline $\mathrm{C} 30-\mathrm{C} 29-\mathrm{C} 28$ & $121.3(3)$ & $\mathrm{C} 1 \mathrm{~L}-\mathrm{C} 1 \mathrm{~K}-\mathrm{H} 1 \mathrm{KA}$ & 110.4 \\
\hline $\mathrm{C} 30-\mathrm{C} 29-\mathrm{H} 29$ & 119.4 & $\mathrm{C} 1 \mathrm{~L}-\mathrm{C} 1 \mathrm{~K}-\mathrm{H} 1 \mathrm{~KB}$ & 110.4 \\
\hline $\mathrm{C} 29-\mathrm{C} 30-\mathrm{H} 30$ & 119.5 & $\mathrm{C} 1 \mathrm{~K}-\mathrm{C} 1 \mathrm{~L}-\mathrm{H} 1 \mathrm{LA}$ & 109.5 \\
\hline $\mathrm{C} 29-\mathrm{C} 30-\mathrm{C} 31$ & $120.9(3)$ & $\mathrm{C} 1 \mathrm{~K}-\mathrm{C} 1 \mathrm{~L}-\mathrm{H} 1 \mathrm{LB}$ & 109.5 \\
\hline $\mathrm{C} 31-\mathrm{C} 30-\mathrm{H} 30$ & 119.5 & $\mathrm{C} 1 \mathrm{~K}-\mathrm{C} 1 \mathrm{~L}-\mathrm{H} 1 \mathrm{LC}$ & 109.5 \\
\hline $\mathrm{C} 30-\mathrm{C} 31-\mathrm{C} 34$ & $120.6(3)$ & $\mathrm{H} 1 \mathrm{LA}-\mathrm{C} 1 \mathrm{~L}-\mathrm{H} 1 \mathrm{LB}$ & 109.5 \\
\hline $\mathrm{C} 32-\mathrm{C} 31-\mathrm{C} 30$ & $117.6(3)$ & $\mathrm{H} 1 \mathrm{LA}-\mathrm{C} 1 \mathrm{~L}-\mathrm{H} 1 \mathrm{LC}$ & 109.5 \\
\hline $\mathrm{C} 32-\mathrm{C} 31-\mathrm{C} 34$ & $121.8(3)$ & $\mathrm{H} 1 \mathrm{LB}-\mathrm{C} 1 \mathrm{~L}-\mathrm{H} 1 \mathrm{LC}$ & 109.5 \\
\hline $\mathrm{Ni}-\mathrm{N} 1-\mathrm{C} 1-\mathrm{C} 2$ & $161.2(2)$ & $\mathrm{C} 10-\mathrm{C} 5-\mathrm{C} 6-\mathrm{C} 7$ & $-1.9(4)$ \\
\hline $\mathrm{Ni}-\mathrm{N} 1-\mathrm{C} 1-\mathrm{C} 5$ & $-23.5(4)$ & $\mathrm{C} 11-\mathrm{C} 8-\mathrm{C} 9-\mathrm{C} 10$ & $178.2(3)$ \\
\hline $\mathrm{Ni}-\mathrm{N} 1-\mathrm{C} 4-\mathrm{C} 3$ & $-166.90(18)$ & $\mathrm{C} 12-\mathrm{C} 3-\mathrm{C} 4-\mathrm{N} 1$ & $-179.7(3)$ \\
\hline $\mathrm{Ni}-\mathrm{N} 1-\mathrm{C} 4-\mathrm{C} 19$ & $3.1(3)$ & $\mathrm{C} 12-\mathrm{C} 3-\mathrm{C} 4-\mathrm{C} 19$ & $13.3(5)$ \\
\hline $\mathrm{Ni}-\mathrm{N} 2-\mathrm{C} 19-\mathrm{C} 4$ & $-6.0(3)$ & $\mathrm{C} 12-\mathrm{C} 13-\mathrm{C} 14-\mathrm{C} 15$ & $-0.1(5)$ \\
\hline $\mathrm{Ni}-\mathrm{N} 2-\mathrm{C} 19-\mathrm{C} 20$ & $169.9(2)$ & $\mathrm{C} 13-\mathrm{C} 12-\mathrm{C} 17-\mathrm{C} 16$ & $3.5(4)$ \\
\hline $\mathrm{Ni}-\mathrm{N} 2-\mathrm{C} 23-\mathrm{C} 22$ & $-170.8(2)$ & $\mathrm{C} 13-\mathrm{C} 14-\mathrm{C} 15-\mathrm{C} 16$ & $2.4(4)$ \\
\hline $\mathrm{Ni}-\mathrm{N} 2-\mathrm{C} 23-\mathrm{C} 24$ & $6.5(3)$ & $\mathrm{C} 13-\mathrm{C} 14-\mathrm{C} 15-\mathrm{C} 18$ & $-177.4(3)$ \\
\hline $\mathrm{Ni}-\mathrm{N} 3-\mathrm{C} 24-\mathrm{C} 23$ & $-7.9(3)$ & $\mathrm{C} 14-\mathrm{C} 15-\mathrm{C} 16-\mathrm{C} 17$ & $-1.7(4)$ \\
\hline $\mathrm{Ni}-\mathrm{N} 3-\mathrm{C} 24-\mathrm{C} 25$ & $163.61(19)$ & $\mathrm{C} 15-\mathrm{C} 16-\mathrm{C} 17-\mathrm{C} 12$ & $-1.3(4)$ \\
\hline $\mathrm{Ni}-\mathrm{N} 3-\mathrm{C} 27-\mathrm{C} 26$ & $-156.8(2)$ & $\mathrm{C} 17-\mathrm{C} 12-\mathrm{C} 13-\mathrm{C} 14$ & $-2.8(4)$ \\
\hline $\mathrm{Ni}-\mathrm{N} 3-\mathrm{C} 27-\mathrm{C} 35$ & $31.3(5)$ & $\mathrm{C} 18-\mathrm{C} 15-\mathrm{C} 16-\mathrm{C} 17$ & $178.1(3)$ \\
\hline $\mathrm{N} 1-\mathrm{Ni}-\mathrm{N} 2-\mathrm{C} 19$ & $6.3(2)$ & $\mathrm{C} 19-\mathrm{N} 2-\mathrm{C} 23-\mathrm{C} 22$ & $-4.1(4)$ \\
\hline $\mathrm{N} 1-\mathrm{Ni}-\mathrm{N} 2-\mathrm{C} 23$ & $173.5(2)$ & $\mathrm{C} 19-\mathrm{N} 2-\mathrm{C} 23-\mathrm{C} 24$ & $173.1(2)$ \\
\hline $\mathrm{N} 1-\mathrm{C} 1-\mathrm{C} 2-\mathrm{C} 3$ & $2.0(3)$ & $\mathrm{C} 19-\mathrm{C} 20-\mathrm{C} 21-\mathrm{C} 22$ & $-1.0(5)$ \\
\hline $\mathrm{N} 1-\mathrm{C} 1-\mathrm{C} 5-\mathrm{C} 6$ & $-48.1(4)$ & $\mathrm{C} 20-\mathrm{C} 21-\mathrm{C} 22-\mathrm{C} 23$ & $0.2(5)$ \\
\hline $\mathrm{N} 1-\mathrm{C} 1-\mathrm{C} 5-\mathrm{C} 10$ & $134.1(3)$ & $\mathrm{C} 21-\mathrm{C} 22-\mathrm{C} 23-\mathrm{N} 2$ & $2.3(4)$ \\
\hline $\mathrm{N} 1-\mathrm{C} 4-\mathrm{C} 19-\mathrm{N} 2$ & $1.7(3)$ & $\mathrm{C} 21-\mathrm{C} 22-\mathrm{C} 23-\mathrm{C} 24$ & $-174.3(3)$ \\
\hline $\mathrm{N} 1-\mathrm{C} 4-\mathrm{C} 19-\mathrm{C} 20$ & $-173.7(3)$ & $\mathrm{C} 22-\mathrm{C} 23-\mathrm{C} 24-\mathrm{N} 3$ & $178.1(3)$ \\
\hline $\mathrm{N} 2-\mathrm{Ni}-\mathrm{N} 1-\mathrm{C} 1$ & $-167.0(3)$ & $\mathrm{C} 22-\mathrm{C} 23-\mathrm{C} 24-\mathrm{C} 25$ & $9.2(5)$ \\
\hline $\mathrm{N} 2-\mathrm{Ni}-\mathrm{N} 1-\mathrm{C} 4$ & $-4.96(19)$ & $\mathrm{C} 23-\mathrm{N} 2-\mathrm{C} 19-\mathrm{C} 4$ & $-172.6(2)$ \\
\hline $\mathrm{N} 2-\mathrm{C} 19-\mathrm{C} 20-\mathrm{C} 21$ & $-0.7(4)$ & $\mathrm{C} 23-\mathrm{N} 2-\mathrm{C} 19-\mathrm{C} 20$ & $3.3(4)$ \\
\hline $\mathrm{N} 2-\mathrm{C} 23-\mathrm{C} 24-\mathrm{N} 3$ & $1.3(3)$ & $\mathrm{C} 23-\mathrm{C} 24-\mathrm{C} 25-\mathrm{C} 26$ & $169.7(3)$ \\
\hline $\mathrm{N} 2-\mathrm{C} 23-\mathrm{C} 24-\mathrm{C} 25$ & $-167.6(3)$ & $\mathrm{C} 23-\mathrm{C} 24-\mathrm{C} 25-\mathrm{C} 28$ & $-11.9(5)$ \\
\hline $\mathrm{N} 3-\mathrm{Ni}-\mathrm{N} 1-\mathrm{C} 1$ & $-177.4(4)$ & $\mathrm{C} 24-\mathrm{N} 3-\mathrm{C} 27-\mathrm{C} 26$ & $2.5(3)$ \\
\hline $\mathrm{N} 3-\mathrm{Ni}-\mathrm{N} 1-\mathrm{C} 4$ & $-15.3(5)$ & $\mathrm{C} 24-\mathrm{N} 3-\mathrm{C} 27-\mathrm{C} 35$ & $-169.4(3)$ \\
\hline $\mathrm{N} 3-\mathrm{Ni}-\mathrm{N} 2-\mathrm{C} 19$ & $-176.1(2)$ & $\mathrm{C} 24-\mathrm{C} 25-\mathrm{C} 26-\mathrm{C} 27$ & $1.1(3)$ \\
\hline $\mathrm{N} 3-\mathrm{Ni}-\mathrm{N} 2-\mathrm{C} 23$ & $-8.8(2)$ & $\mathrm{C} 24-\mathrm{C} 25-\mathrm{C} 28-\mathrm{C} 29$ & $-55.6(4)$ \\
\hline $\mathrm{N} 3-\mathrm{C} 24-\mathrm{C} 25-\mathrm{C} 26$ & $0.5(3)$ & $\mathrm{C} 24-\mathrm{C} 25-\mathrm{C} 28-\mathrm{C} 33$ & $123.9(3)$ \\
\hline $\mathrm{N} 3-\mathrm{C} 24-\mathrm{C} 25-\mathrm{C} 28$ & $178.9(3)$ & $\mathrm{C} 25-\mathrm{C} 26-\mathrm{C} 27-\mathrm{N} 3$ & $-2.2(3)$ \\
\hline $\mathrm{N} 3-\mathrm{C} 27-\mathrm{C} 35-\mathrm{C} 36$ & $-152.3(3)$ & $\mathrm{C} 25-\mathrm{C} 26-\mathrm{C} 27-\mathrm{C} 35$ & $169.6(3)$ \\
\hline
\end{tabular}




$\mathrm{N} 3-\mathrm{C} 27-\mathrm{C} 35-\mathrm{C} 40$
$\mathrm{~N} 4-\mathrm{Ni}-\mathrm{N} 1-\mathrm{C} 1$
$\mathrm{~N} 4-\mathrm{N}-\mathrm{N} 1-\mathrm{C} 4$
$\mathrm{~N} 4-\mathrm{Ni}-\mathrm{N} 2-\mathrm{C} 19$
$\mathrm{~N} 4-\mathrm{Ni}-\mathrm{N} 2-\mathrm{C} 23$
$\mathrm{C} 1-\mathrm{N} 1-\mathrm{C} 4-\mathrm{C} 3$
$\mathrm{C} 1-\mathrm{N} 1-\mathrm{C} 4-\mathrm{C} 19$
$\mathrm{C} 1-\mathrm{C} 2-\mathrm{C} 3-\mathrm{C} 4$
$\mathrm{C} 1-\mathrm{C} 2-\mathrm{C} 3-\mathrm{C} 12$
$\mathrm{C} 1-\mathrm{C} 5-\mathrm{C} 6-\mathrm{C} 7$
$\mathrm{C} 1-\mathrm{C} 5-\mathrm{C} 10-\mathrm{C} 9$
$\mathrm{C} 2-\mathrm{C} 1-\mathrm{C} 5-\mathrm{C} 6$
$\mathrm{C} 2-\mathrm{C} 1-\mathrm{C} 5-\mathrm{C} 10$
$\mathrm{C} 2-\mathrm{C} 3-\mathrm{C} 4-\mathrm{N} 1$
$\mathrm{C} 2-\mathrm{C} 3-\mathrm{C} 4-\mathrm{C} 19$
$\mathrm{C} 2-\mathrm{C} 3-\mathrm{C} 12-\mathrm{C} 13$
$\mathrm{C} 2-\mathrm{C} 3-\mathrm{C} 12-\mathrm{C} 17$
$\mathrm{C} 3-\mathrm{C} 4-\mathrm{C} 19-\mathrm{N} 2$
$\mathrm{C} 3-\mathrm{C} 4-\mathrm{C} 19-\mathrm{C} 20$
$\mathrm{C} 3-\mathrm{C} 12-\mathrm{C} 13-\mathrm{C} 14$
$\mathrm{C} 3-\mathrm{C} 12-\mathrm{C} 17-\mathrm{C} 16$
$\mathrm{C} 4-\mathrm{N} 1-\mathrm{C} 1-\mathrm{C} 2$
$\mathrm{C} 4-\mathrm{N} 1-\mathrm{C} 1-\mathrm{C} 5$
$\mathrm{C} 4-\mathrm{C} 3-\mathrm{C} 12-\mathrm{C} 13$
$\mathrm{C} 4-\mathrm{C} 3-\mathrm{C} 12-\mathrm{C} 17$
$\mathrm{C} 4-\mathrm{C} 19-\mathrm{C} 20-\mathrm{C} 21$
$\mathrm{C} 5-\mathrm{C} 1-\mathrm{C} 2-\mathrm{C} 3$
$\mathrm{C} 5-\mathrm{C} 6-\mathrm{C} 7-\mathrm{C} 8$
$\mathrm{C} 6-\mathrm{C} 5-\mathrm{C} 10-\mathrm{C} 9$
$\mathrm{C} 6-\mathrm{C} 7-\mathrm{C} 8-\mathrm{C} 9$
$\mathrm{C} 6-\mathrm{C} 7-\mathrm{C} 8-\mathrm{C} 11$
$\mathrm{C} 7-\mathrm{C} 8-\mathrm{C} 9-\mathrm{C} 10$
$\mathrm{C} 8-\mathrm{C} 9-\mathrm{C} 10-\mathrm{C} 5$

$32.6(4)$

$1.0(3)$

$163.01(19)$

$-85.1(5)$

$82.1(5)$

0.7 (3)

$170.7(2)$

$-1.5(3)$

178.7 (3)

$-179.7(3)$

$179.3(2)$

$126.5(3)$

$-51.2(4)$

$0.5(3)$

$-166.5(3)$

43.0 (4)

$-135.9(3)$

$168.3(3)$

$-7.0(5)$

$178.2(3)$

$-177.5(3)$

$-1.7(3)$

$173.7(2)$

$-136.8(3)$

44.3 (4)

$174.3(3)$

$-173.3(3)$

$0.8(4)$

$1.5(4)$

$0.8(4)$

$-178.7(3)$

$-1.3(4)$

0.1 (4)
$178.4(3)$

$-178.8(3)$

122.5 (3)

-58.0 (4)

37.1 (4)

-138.0 (3)

-173.4 (2)

-1.8 (3)

-176.9 (3)

178.1 (3)

$-177.3(3)$

0.9 (4)

0.7 (4)

-0.2 (4)

$179.5(3)$

-0.3 (5)

0.0 (5)

-1.1 (4)

-180.0 (3)

$-1.6(5)$

2.9 (4)

3.4 (5)

-175.8 (3)

-2.0 (5)

$-1.1(5)$

-1.6 (4)

$177.1(3)$

178.1 (7)

$72.3(8)$

$169.8(7)$

177 (2)

130 (3)

148 (3) 